\title{
High energy physics in the atmosphere: phenomenology of cosmic ray air showers
}

\author{
Luis Anchordoqui ${ }^{\mathrm{a}, *}$, María Teresa Dova ${ }^{\mathrm{b}}$, \\ Analisa Mariazzi ${ }^{\mathrm{b}}$, Thomas McCauley ${ }^{\mathrm{a}}$, \\ Thomas Paul ${ }^{\mathrm{a}}$, Stephen Reucroft ${ }^{\mathrm{a}}$, John Swain ${ }^{\mathrm{a}}$ \\ a Department of Physics, Northeastern University, Boston, MA 02115, USA \\ b Departamento de Física, Universidad Nacional de La Plata, CC67 La Plata (1900), Argentina
}

Received 13 July 2004; accepted 22 July 2004

Available online 15 September 2004

\begin{abstract}
The properties of cosmic rays with energies above $10^{6} \mathrm{GeV}$ have to be deduced from the spacetime structure and particle content of the air showers which they initiate. In this review we summarize the phenomenology of these giant air showers. We describe the hadronic interaction models used to extrapolate results from collider data to ultra high energies, and discuss the prospects for insights into forward physics at the LHC. We also describe the main electromagnetic processes that govern the longitudinal shower evolution, as well as the lateral spread of particles. Armed with these two principal shower ingredients and motivation from the underlying physics, we provide an overview of some of the different methods proposed to distinguish primary species. The properties of neutrino interactions and the potential of forthcoming experiments to isolate deeply penetrating showers from baryonic cascades are also discussed. We finally venture into a terra incognita endowed with TeV-scale gravity and explore anomalous neutrino-induced showers.
\end{abstract}

(C) 2004 Elsevier Inc. All rights reserved.

PACS: 96.40.z; 13.85.Tp; 13.15.+g

Keywords: Extensive air showers; Cosmic rays

\footnotetext{
${ }^{*}$ Corresponding author. Fax: +16173737835.

E-mail address: 1.anchordoqui@neu.edu (L. Anchordoqui).
} 


\section{Introduction}

\subsection{Why another cosmic ray review?}

Some 40 years after the discovery of ultra high energy cosmic rays [1], fundamental questions regarding their origin and nature lack definitive answers. The highest primary energy measured thus far is $E \sim 10^{11.5} \mathrm{GeV}$ [2], corresponding to a nucleon-nucleon center-of-mass energy $\sqrt{s} \sim 10^{5.9} \mathrm{GeV} / \sqrt{A}$, where $A$ is the mass number of the primary particle. The existence of these particles, the most energetic known in the universe, challenges our current understanding of physics. From the perspective of astrophysics, one must identify where and how in the universe these particles obtain such high energies. A failure to uncover such mechanisms may lead one to postulate new physics to explain the phenomenon. From the perspective of particle physics, ultra high energy cosmic ray interactions are orders of magnitude beyond what can be achieved in current (and future) terrestrial collider experiments and may open a window to energy and kinematic regions previously unexplored in the study of fundamental interactions. From both perspectives, the tantalizing possibility of new physics that may be found in the study of ultra high energy cosmic rays continues to motivate current and future cosmic ray experiments.

The literature abounds in reviews of experimental techniques for detection of cosmic ray air showers [3-9], as well as overviews of the physics of cosmic ray propagation and possible astrophysical and exotic origins [10-18]. This review follows a somewhat different path, focusing exclusively on cosmic ray phenomenology from the top of the atmosphere to the Earth's surface. The topics covered are viewed from the perspective of particle physics, and the reader is assumed only to possess a basic background in this field. We hope this article can provide a sort of bridge for high energy physicists interested in exploring some of the challenges facing upcoming ground- and space-based cosmic ray observatories.

\subsection{Cosmic ray observations}

For primary energy $E \gtrsim 1 \mathrm{GeV}$ the observed cosmic ray flux can be described by a series of power laws with the flux falling about three orders of magnitude for each decade increase in energy. In the decade centered at $\left.\sqrt{s}\right|_{\text {knee }} \sim 10^{3.4} \mathrm{GeV} / \sqrt{A}$, the spectrum steepens from $E^{-2.7}$ to $E^{-3.0}$, forming the feature commonly known as "the knee." The spectrum steepens further to $E^{-3.3}$ above $\left.\sqrt{s}\right|_{\text {dip }} \sim 10^{4.5} \mathrm{GeV} / \sqrt{A}$, and then flattens to $E^{-2.7}$ at $\left.\sqrt{s}\right|_{\text {ankle }} \sim 10^{5.1} \mathrm{GeV} / \sqrt{A}$, forming a feature known as "the ankle" [19]. Within the statistical uncertainty of existing data, which is large for $E>10^{11} \mathrm{GeV}$, the tail of the spectrum is consistent with a simple extrapolation at that slope to the highest energies. Thus far, for Earth-based accelerators, the record holder for collisions with the highest energy per nucleon is the Tevatron, which countercirculates protons and antiprotons with $\sqrt{s} \simeq 1.8 \mathrm{TeV}$. This center-of-mass energy corresponds closely to that at the knee. The Large Hadron Collider (LHC), now under construction at CERN, will collide protons with protons at $\sqrt{s} \simeq 14 \mathrm{TeV}$. This impressive energy is still about a factor of 50 smaller than the 
center-of-mass energy of the highest energy cosmic ray so far observed, assuming $A=1$.

For primary cosmic ray energies above $10^{5} \mathrm{GeV}$, the flux becomes so low that direct detection of the primary using devices in or above the upper atmosphere is, for all practical purposes, impossible. Fortunately, in such cases the primary particle has enough energy to initiate a particle cascade in the atmosphere large enough that the products are detectable at ground. There are several techniques which can be employed in detecting these extensive air showers (EAS), ranging from sampling of particles in the cascade to measurements of fluorescence, Cerenkov or radio emissions produced by the shower.

The most commonly used detection method involves sampling the shower front at a given altitude using an array of sensors spread over a large area. Sensors, such as plastic scintillators or Čerenkov detectors are used to infer the particle density and the relative arrival times of the shower front at different locations; from this, one can estimate the energy and direction of the primary cosmic ray. The spacing between stations determines the energy threshold for a vertical shower. The muon content is usually sought either by exploiting the signal timing in the surface sensors or by employing dedicated detectors which are shielded from the electromagnetic shower component. Inferring the primary energy from energy deposits at the ground is not completely straightforward, and involves proper modeling of both the detector response and the physics of the first few cascade generations. This second point is particularly subtle and will be the main subject of Section 2 .

Another highly successful air shower detection method involves measurement of the longitudinal development of the cascade by sensing the fluorescence light produced via interactions of the charged particles in the atmosphere. As an extensive air shower develops, it dissipates much of its energy by exciting and ionizing air molecules along its path. Excited nitrogen molecules fluoresce producing radiation in the $300-400 \mathrm{~nm}$ ultraviolet range, to which the atmosphere is quite transparent. Under favorable atmospheric conditions EAS can be detected at distances as large as $20 \mathrm{~km}$, about two attenuation lengths in a standard desert atmosphere at ground level, though observations can only be made on clear moonless nights, yielding a duty cycle of about $10 \%$. The shower development appears as a rapidly moving spot of light whose angular motion depends on both the distance and the orientation of the shower axis. The integrated light signal is proportional to the total energy deposited in the atmosphere. Systematic errors can arise from a variety of sources, including uncertainties in the nitrogen fluorescence induced by the particle beam [20,21], as well as uncertainties in the atmospheric conditions at the time the fluorescence measurements are taken [22].

The first measurements of ultra high energy cosmic rays were carried out by Linsley at Volcano Ranch $\left(35^{\circ} 09^{\prime} \mathrm{N}, 106^{\circ} 47^{\prime} \mathrm{W}\right)$ in the late 1950 's [23] using an array of scintillation counters. More recent experiments using surface detection techniques include Haverah Park in England $\left(53^{\circ} 58^{\prime} \mathrm{N}, 1^{\circ} 38^{\prime} \mathrm{W}\right)$ [24], Yakutsk in Russia $\left(62^{\circ} \mathrm{N}\right.$, $130^{\circ} \mathrm{E}$ ) [25], the Sydney University Giant Airshower Recorder (SUGAR) in Australia $\left(30^{\circ} 32^{\prime} \mathrm{S}, 149^{\circ} 43^{\prime} \mathrm{E}\right)$ [26], and the Akeno Giant Air Shower Array (AGASA), about $100 \mathrm{~km}$ west of Tokyo $\left(38^{\circ} 47^{\prime} \mathrm{N}, 138^{\circ} 30^{\prime} \mathrm{E}\right)[27,28]$. The fluorescence method has been used by the Fly's Eye experiment [29,30], as well as its up-scoped 
descendant High Resolution Fly's Eye experiment (HiRes) [31], operating at the Dugway proving ground in the Utah desert $\left(40^{\circ} \mathrm{N}, 112^{\circ} \mathrm{W}\right)$.

Over the next few years, the best observations of the extreme end of the cosmic ray spectrum will be made by the Pierre Auger Observatory (PAO) [32], which is currently operational in Malargüe, Argentina $\left(35^{\circ} 12^{\prime} \mathrm{S}, 69^{\circ} 12^{\prime} \mathrm{W}\right)$ and is in the process of growing to its final size of $3000 \mathrm{~km}^{2}$. A twin site is pending for the Northern hemisphere, and together the two observatories will have an acceptance of $14,000 \mathrm{~km}^{2} \mathrm{sr}$ above $10^{10} \mathrm{GeV}$ for zenith angles below $60^{\circ}$. The PAO works in a hybrid mode, and when complete will comprise 24 fluorescence detectors overlooking a ground array of 1600 water Čerenkov detectors. During clear, dark nights, events are simultaneously observed by fluorescence light and particle detectors, allowing powerful reconstruction and cross-calibration techniques. Simultaneous observations of showers using two distinct detector methods will also help to control the systematic errors that have plagued cosmic ray experiments to date. Moreover, each site of PAO reaches $\sim 15 \mathrm{~km}^{3}$ we sr of target mass around $10^{10} \mathrm{GeV}$ [33], which is comparable to dedicated neutrino detectors being planned. ${ }^{1}$ This renders PAO a neutrino telescope operating in an energy regime complimentary to existing and upcoming facilities. The characteristics of neutrino-induced showers are discussed in Section 5.

Space-based experiments are also in the offing, the most thoroughly planned being the Extreme Universe Space Observatory (EUSO) [34,35]. EUSO comprises a single fluorescence eye, and is scheduled to fly aboard the International Space Station for more than 3 years. After taking account of the $10 \%$ duty cycle, this experiment will image a vast volume of $750 \mathrm{~km}^{3}$ we sr.

In recent years, a somewhat confused picture vis-à-vis the energy spectrum and arrival direction distribution has been emerging. Since 1998, the AGASA Collaboration has consistently reported [36,37] a continuation of the spectrum beyond the expected Greisen-Zatsepin-Kuzmin (GZK) cutoff [38,39], which should arise at about $10^{10.9} \mathrm{GeV}$ if cosmic ray sources are at cosmological distances. This theoretical feature of the spectrum is mainly a consequence of interactions of the primary cosmic ray with the microwave background radiation. In contrast, the most recent results from HiRes [40] describe a spectrum which is consistent with the expected GZK feature. The discrepancy between the two estimated fluxes is shown in Fig. 1. Several analyses were done in trying to understand this difference. In particular, since the quoted systematic errors in the energy estimate are in the neighbourhood of $30 \%$, it was argued [42] that if the AGASA Collaboration overestimates their energies by $30 \%$ and the HiRes Collaboration underestimates them by about the same amount, then the two spectra can be brought into reasonable agreement within statistical errors.

Although there seems to be a remarkable agreement among experiments on predictions about isotropy on large scale structure $[43,44]$, this is certainly not the case when considering the two-point correlation function on a small angular scale. The AGASA Collaboration reports observations of event clusters which have a chance probability smaller than $1 \%$ to arise from a random distribution $[45,46]$. Far from confirming the

\footnotetext{
${ }^{1}$ we $\equiv$ water equivalent.
} 


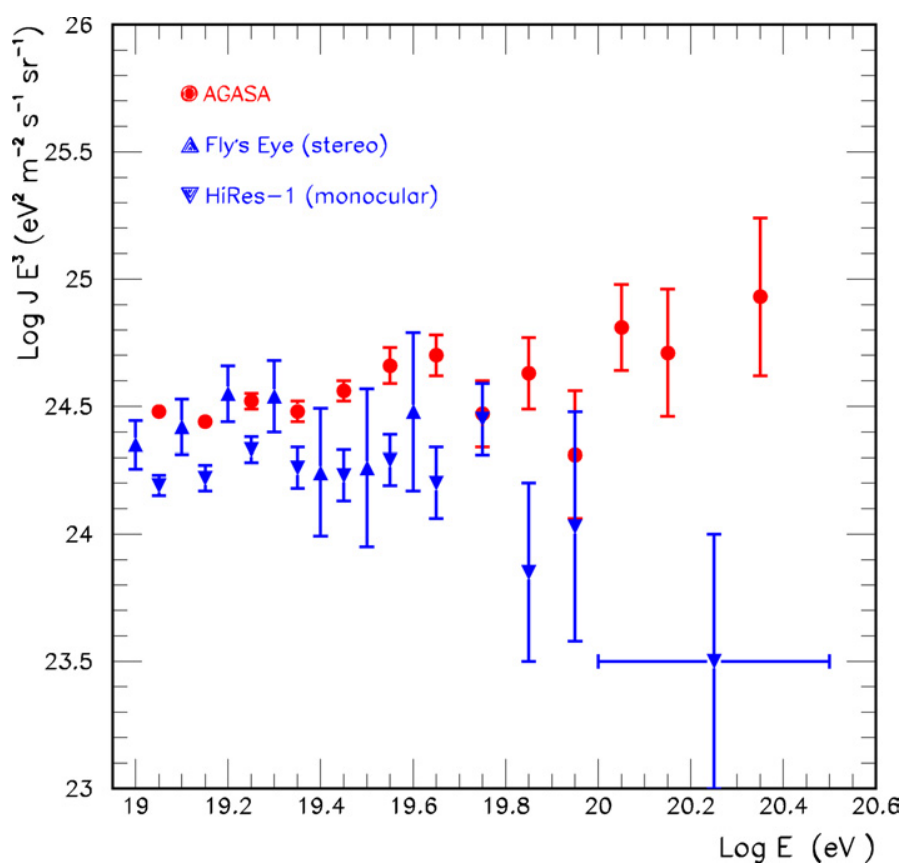

Fig. 1. Upper end of the cosmic ray energy spectrum as observed by AGASA [37] and HiRes [40]/Fly's Eye [41].

AGASA results, the recent analysis reported by the HiRes Collaboration showed that their data are consistent with no clustering among the highest energy events [47,48]. The discovery of such clusters would be a tremendous breakthrough for the field, but the case for them is not yet proved. Special care must be taken when computing the statistical significance in such an analysis. In particular, it is important to define the search procedure a priori in order to ensure one does not inadvertently perform "trials" by studying the data before deciding upon the cuts. Very recently, with the aim of avoiding accidental bias on the number of trials performed in selecting the angular bin, the original claim of the AGASA Collaboration [45] was re-examined considering only those events observed after the original claim [49]. This study showed that the evidence for clustering in the AGASA data set is weaker than was previously supposed, and is consistent with the hypothesis of isotropically distributed arrival directions.

The confusing experimental situation regarding the GZK feature and the smallscale clustering in the distribution of arrival direction should be resolved in the near future by the PAO, which will provide not only a data set of unprecedented size, but also the machinery for controlling some of the more problematic systematic uncertainties. As we will discuss in this review, however, the task of identifying primary species is more challenging. The rest of this article is organized as follows. In Section 2, we describe the phenomenology of hadronic interactions with the goal of providing an overview of the main systematic uncertainties hindering the determination of 
the primary energy from observations at the ground. In the following section, we discuss the electromagnetic processes responsible for generating the great majority of particles in the shower. Armed with these two principal shower ingredients, we then discuss observables that are accessible to experiment. Next, in Section 4, we describe how these observables are used to infer the primary composition. In Section 5, we summarize properties of neutrino interactions and discuss observables in the deeply penetrating showers they produce. Since the expected rate of such events is very low, any enhancement of the cross section due to physics beyond the electroweak scale should be evident in this channel. At the end we allow ourselves to venture into speculative territory and discuss the experimental signatures of neutrino interactions in scenarios with $\mathrm{TeV}$-scale gravity. Before getting underway, we first briefly summarize the main features of the atmospheric "calorimeter."

\subsection{Nature's calorimeter}

Unlike man-made calorimeters, the atmosphere is a calorimeter whose properties vary in a predictable way with altitude, and in a relatively unpredictable way with time. Beginning with the easier of the two variations, we note that the density and pressure depend strongly on the height, while the temperature does not change by more than about $30 \%$ over the range $0-100 \mathrm{~km}$ above sea level. Therefore, we can get a reasonable impression of the density variation by assuming an isothermal atmosphere, in which case the density $\rho_{\text {atm }}(h) \approx \rho_{0} \mathrm{e}^{-h / h_{0}}$, where $\rho_{0} \approx 1.225 \mathrm{~kg} / \mathrm{m}^{3}$ and $h_{0}=R T /(\mu \mathrm{g}) \approx 8.4 \mathrm{~km}$ is known as the scale-height of the atmosphere, $R$ being the ideal gas constant, $\mu$ the average molecular weight of air, $g$ the acceleration due to gravity, and $T \approx 288 \mathrm{~K}$. Of course, reading out such a natural calorimeter is complicated by the effects of varying aerosol and molecular attenuation and scattering.

The quantity that most intuitively describes the varying density of the atmospheric medium is the vertical atmospheric depth, $X_{v}(h)=\int_{h}^{\infty} \rho_{\text {atm }}(z) \mathrm{d} z$, where $z$ is the height. However, the quantity most relevant in air shower simulations is the slant depth, $X$, which defines the actual amount of air traversed by the shower. The variation of the slant depth with zenith angle is shown in Fig. 2. If the Earth curvature is not taken into

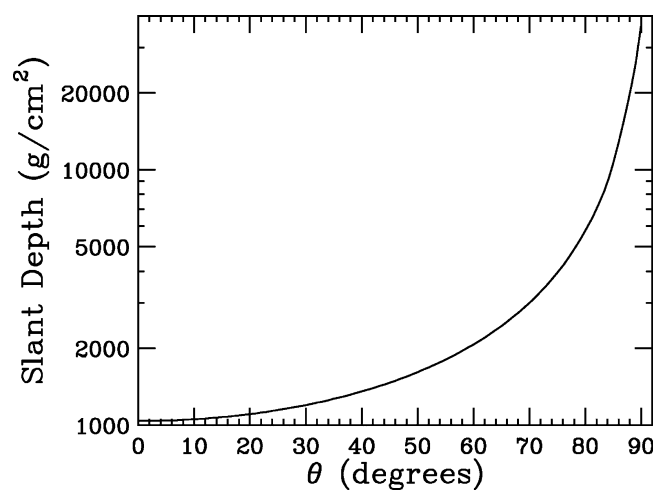

Fig. 2. Slant depths corresponding to various zenith angles $\theta$ considering the curvature of the Earth. 
account, then $X=X_{v}(h) / \cos \theta$, where $\theta$ is the zenith angle of the shower axis. For $\theta \lesssim 80^{\circ}$, the error associated with this approximation is less than $4 \%$.

The atmospheric medium is endowed with a magnetic field. In general, the geomagnetic field is described by three parameters, its strength $|\vec{B}|$, its inclination $l$, and its declination $\delta$. The inclination is defined as the angle between the local horizontal plane and the $\vec{B}$-field. The declination is defined as the angle between the horizontal component of the field $B_{\perp}$ (i.e., perpendicular to the arrival direction of the air shower) and the geographical North (direction of the local meridian). The angle $\imath$ is positive when $\vec{B}$ points downward and $\delta$ is positive when $B_{\perp}$ is inclined towards the East.

\section{Hadronic processes}

Uncertainties in hadronic interactions at ultra high energies constitute one of the most problematic sources of systematic error in analysis of air showers. This section will explain the two principal schools of thought for extrapolating collider data to ultra high energies. We start with a general description of $p p$ collisions within the eikonal model. Next, we consider the specific case of hadronic interactions in the atmosphere, and discuss the most widely used Monte Carlo codes. Finally, we study the potential of present and future accelerators to produce data valuable for understanding extensive air shower physics. Particular emphasis is placed on measurements of interaction processes at extreme forward directions and the cross sections for diffractive interactions.

\subsection{Low- $p_{T}$ jet physics beyond collider energies}

Soft multiparticle production with small transverse momenta with respect to the collision axis is a dominant feature of most hadronic events at center-of-mass energies $10 \mathrm{GeV}<\sqrt{s}<50 \mathrm{GeV}$ (see e.g. [50,51]). Despite the fact that strict calculations based on ordinary QCD perturbation theory are not feasible, there are some phenomenological models that successfully take into account the main properties of the soft diffractive processes. These models, inspired by $1 / N$ QCD expansion are also supplemented with generally accepted theoretical principles like duality, unitarity, Regge behavior, and parton structure. The interactions are no longer described by single particle exchange, but by highly complicated modes known as Reggeons. Up to about $50 \mathrm{GeV}$, the slow growth of the cross section with $\sqrt{s}$ is driven by a dominant contribution of a special Reggeon, the Pomeron.

At higher energies, semihard interactions arising from the hard scattering of partons that carry only a very small fraction of the momenta of their parent hadrons can compete successfully with soft processes [52-59]. These semihard interactions lead to the minijet phenomenon, i.e., jets with transverse energy $\left(E_{T}=\left|p_{T}\right|\right)$ much smaller than the total center-of-mass energy. Unlike soft processes, this low- $p_{T}$ jet physics can be computed in perturbative QCD. The parton-parton minijet cross section is given by 


$$
\sigma_{\mathrm{QCD}}\left(s, p_{T}^{\text {cutoff }}\right)=\sum_{i, j} \int \frac{\mathrm{d} x_{1}}{x_{1}} \int \frac{\mathrm{d} x_{2}}{x_{2}} \int_{Q_{\min }^{2}}^{\hat{s} / 2} \mathrm{~d}|\hat{t}| \frac{\mathrm{d} \hat{\sigma}_{i j}}{\mathrm{~d}|\hat{t}|} x_{1} f_{i}\left(x_{1},|\hat{t}|\right) x_{2} f_{j}\left(x_{2},|\hat{t}|\right),
$$

where $x_{1}$ and $x_{2}$ are the fractions of the momenta of the parent hadrons carried by the partons which collide, $\mathrm{d} \hat{\sigma}_{i j} / \mathrm{d}|\hat{t}|$ is the cross section for scattering of partons of types $i$ and $j$ according to elementary QCD diagrams, $f_{i}$ and $f_{j}$ are parton distribution functions (pdf's), $\hat{s}=x_{1} x_{2} s$ and $-\hat{t}=\hat{s}\left(1-\cos \vartheta^{*}\right) / 2=Q^{2}$ are the Mandelstam variables for this parton-parton process, and the sum is over all parton species. Here,

$$
p_{T}=E_{\text {jet }}^{\mathrm{lab}} \sin \vartheta_{\text {jet }}=\frac{\sqrt{\hat{s}}}{2} \sin \vartheta^{*}
$$

and

$$
p_{\|}=E_{\text {jet }}^{\mathrm{lab}} \cos \vartheta_{\text {jet }},
$$

where $E_{\text {jet }}^{\text {lab }}$ is the energy of the jet in the lab frame, $\vartheta_{\text {jet }}$ the angle of the jet with respect to the beam direction in the lab frame, and $\vartheta^{*}$ is the angle of the jet with respect to the beam direction in the center-of-mass frame of the elastic parton-parton collision. This implies that for small $\vartheta^{*}, p_{T}^{2} \approx Q^{2}$. The integration limits satisfy $Q_{\min }^{2}<|\hat{t}|<$ $\hat{s} / 2$, with $Q_{\min }$ the minimal momentum transfer.

A first source of uncertainty in modeling cosmic ray interactions at ultra high energy is encoded in the extrapolation of the measured parton densities several orders of magnitude down to low $x$. Primary protons that impact on the upper atmosphere with energy $\gtrsim 10^{11} \mathrm{GeV}$, yield partons with $x \equiv 2 p_{\|}^{*} / \sqrt{s} \gtrsim m_{\pi} / \sqrt{s} \sim 10^{-7}$, whereas current data on quark and gluon densities are only available for $x \gtrsim 10^{-4}$ to within an experimental accuracy of $3 \%$ for $Q^{2} \approx 20 \mathrm{GeV}^{2}$ [60]. In Fig. 3, we show the region of the $x-Q^{2}$ plane probed by H1, ZEUS, ${ }^{2}$ and fixed target experiments. Moreover, application of HERA data to baryonic cosmic rays assumes universality of the pdf's. The QCD factorization conjecture, which is essentially equivalent to the InglemanSchlein model [61], posits that the parton-parton minijet cross section of Eq. (1) can always be written in a form which factorizes the parton densities and the hard interaction processes irrespective of the order in perturbation theory and the particular hard process. This conjecture holds in the limit $Q^{2} \gg \Lambda_{\mathrm{QCD}}$, where $\Lambda_{\mathrm{QCD}} \sim$ $200 \mathrm{MeV}$ is the QCD renormalization scale. However, a severe breakdown of the factorization conjecture has been observed when using the pdf's obtained by the HERA experiments to predict diffractive jet production in hadron-hadron interactions at the Tevatron [62].

For large $Q^{2}$ and not too small $x$, the Dokshitzer-Gribov-Lipatov-Altarelli-Parisi (DGLAP) equations [63-66]

$$
\frac{\partial}{\partial \ln Q^{2}}\left(\begin{array}{c}
q\left(x, Q^{2}\right) \\
g\left(x, Q^{2}\right)
\end{array}\right)=\frac{\alpha_{s}\left(Q^{2}\right)}{2 \pi}\left(\begin{array}{c}
P_{q q} P_{q g} \\
P_{g q} P_{g g}
\end{array}\right) \otimes\left(\begin{array}{c}
q\left(x, Q^{2}\right) \\
g\left(x, Q^{2}\right)
\end{array}\right)
$$

\footnotetext{
${ }^{2}$ In the $p e^{ \pm}$storage ring HERA at DESY, $27.6 \mathrm{GeV} e^{ \pm}$, s are collided on $820 \mathrm{GeV} p$ 's, and data are recorded by two experiments, $\mathrm{H} 1$ and ZEUS. These collisions correspond to $\sqrt{s} \approx 300 \mathrm{GeV}$, or equivalently a lepton energy $\approx 47 \mathrm{TeV}$ in the proton rest frame.
} 


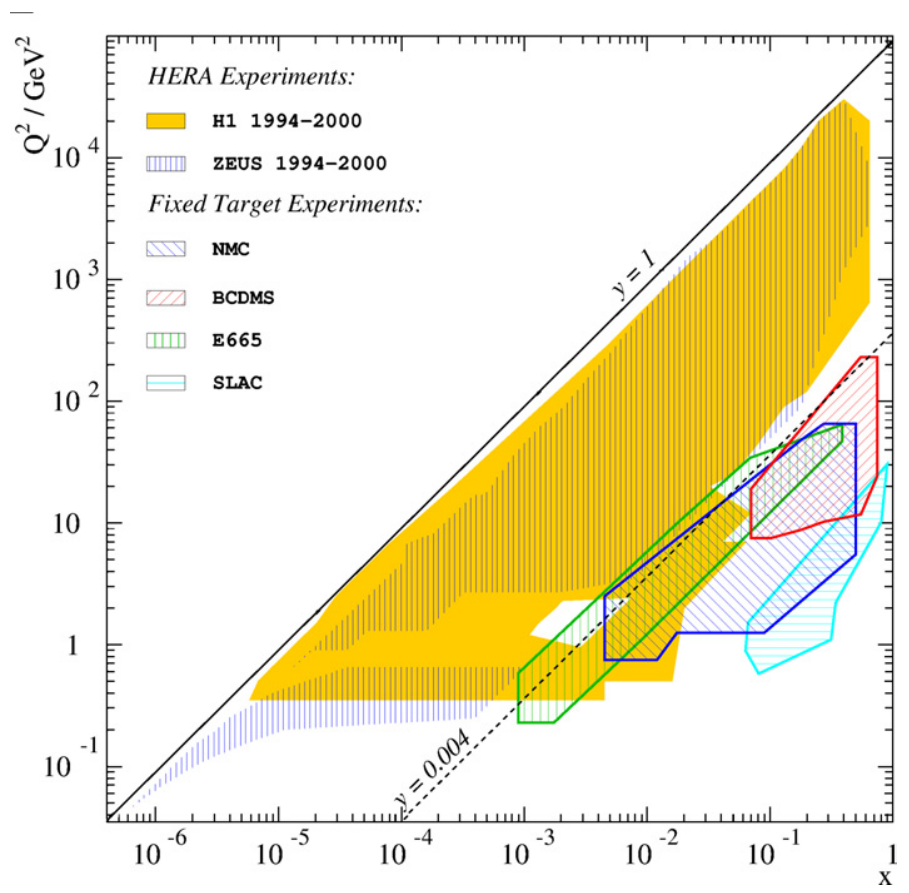

Fig. 3. Kinematic $x-Q^{2}$ plane accessible to the H1 and ZEUS experiments at HERA and the region accessible to fixed-target experiments. The inelasticity $y=\left(1-\cos \vartheta^{*}\right) / 2$ is also shown. This figure is courtesy of Max Klein.

successfully predict the $Q^{2}$ dependence of the quark and gluon densities ( $q$ and $g$, respectively). Here, $\alpha_{s}=g_{s}^{2} /(4 \pi)$, with $g_{s}$ the strong coupling constant. The splitting functions $P_{i j}$ indicate the probability of finding a daughter parton $i$ in the parent parton $j$ with a given fraction of parton $j$ momentum. This probability will depend on the number of splittings allowed in the approximation. In the double-leading-logarithmic approximation, $\operatorname{limit}\left[\ln (1 / x), \ln \left(Q^{2} / \Lambda_{\mathrm{OCD}}^{2}\right)\right] \rightarrow \infty$, the DGLAP equations predict a steeply rising gluon density, $x g \sim x^{-0.4}$, which dominates the quark density at low $x$, in agreement with experimental results obtained with the HERA collider [67]. Specifically, as can be seen in Fig. 4, HERA data are found to be consistent with a power law, $x g\left(x, Q^{2}\right) \sim x^{-\Delta_{H}}$, with an exponent $\Delta_{\mathrm{H}}$ between 0.3 and 0.4 [68]. However, it is easily seen using geometrical arguments that the rapid growth of the gluon density at low $x$ would eventually require corrections to the evolution equations [69].

The high energy minijet cross section is then determined by the small- $x$ behavior of the parton distributions or, rather, by that of the dominant gluon distribution (via the lower limits of the $x_{1}, x_{2}$ integrations)

$$
\sigma_{\mathrm{QCD}}\left(s, p_{T}^{\text {cutoff }}\right) \approx \int \frac{\mathrm{d} x_{1}}{x_{1}} \int \frac{\mathrm{d} x_{2}}{x_{2}} \int_{Q_{\text {min }}^{2}}^{\hat{s} / 2} \mathrm{~d}|\hat{t}| \frac{\mathrm{d} \hat{\sigma}}{\mathrm{d}|\hat{t}|} x_{1} g\left(x_{1},|\hat{t}|\right) x_{2} g\left(x_{2},|\hat{t}|\right) .
$$

A naïve estimate of the $\sigma_{\mathrm{QCD}}$ behavior at high energies can be obtained via extrapolation of $x g \propto x^{-\Delta_{H}}$ to small $x$ in Eq. (5). Within this approximation it is 


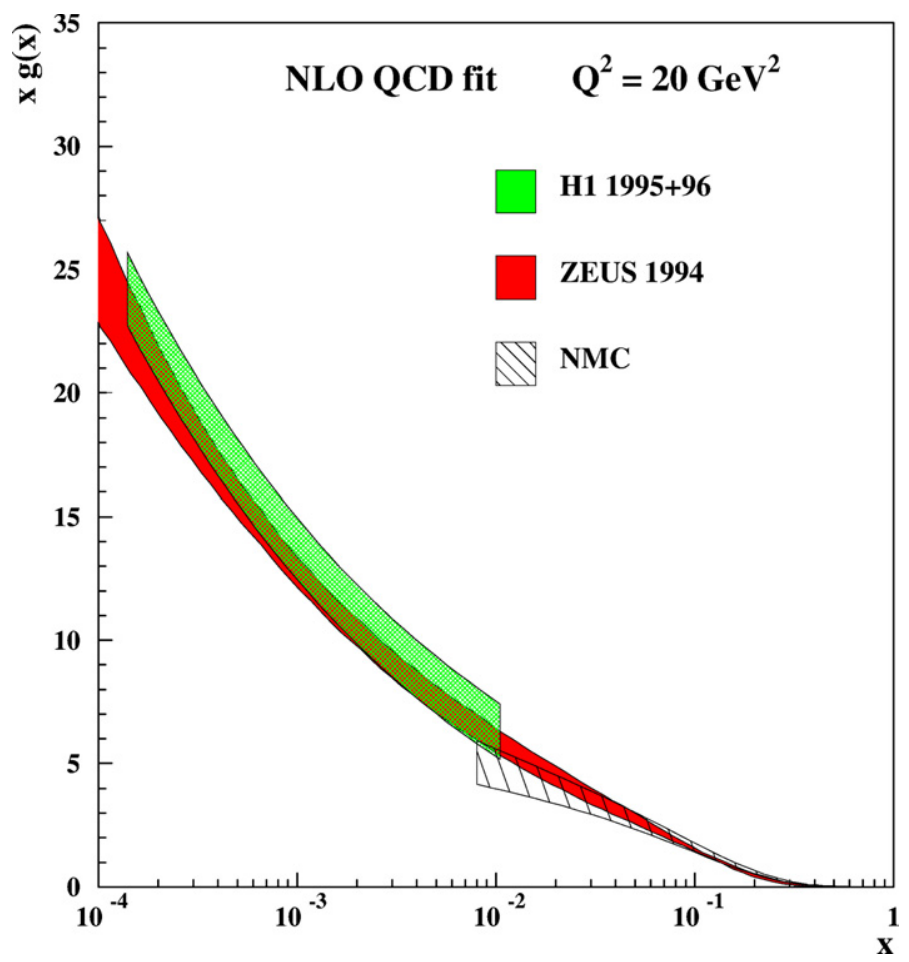

Fig. 4. Gluon density for $x>10^{-4}$ and $Q^{2}=20 \mathrm{GeV}^{2}$, as measured by H1, ZEUS, and NMC experiments.

sufficiently accurate to keep only the leading contribution of the differential cross section for $g g$ scattering (i.e., $\mathrm{d} \hat{\sigma} / \mathrm{d}|\hat{t}| \propto|\hat{t}|^{-2}$ ), and so Eq. (5) becomes [70]

$$
\sigma_{\mathrm{QCD}}(s) \propto \int_{2 Q_{\min }^{2} / s}^{1} \frac{\mathrm{d} x_{1}}{x_{1}} x_{1}^{-\Delta_{\mathrm{H}}} \int_{2 Q_{\text {min }}^{2} / s}^{1} \frac{\mathrm{d} x_{2}}{x_{2}} x_{2}^{-\Delta_{\mathrm{H}}} \sim s^{\Lambda_{\mathrm{H}}} \ln \left(s / s_{0}\right),
$$

where $s_{0}$ is a normalization constant. One caveat is that the inclusive QCD cross section given in Eq. (6) is a Born approximation, and therefore automatically violates unitarity.

The procedure of calculating the inelastic cross section from inclusive cross sections is known as unitarization. In the eikonal model [71-74] of high energy hadron-hadron scattering, the unitarized (elastic, inelastic, and total) cross section, assuming a real eikonal function, is given by:

$$
\begin{aligned}
& \sigma_{\text {el }}=\int \mathrm{d}^{2} \vec{b}\left\{1-\exp \left[-\chi_{\text {soft }}(s, \vec{b})-\chi_{\text {hard }}(s, \vec{b})\right]\right\}^{2}, \\
& \sigma_{\text {inel }}=\int \mathrm{d}^{2} \vec{b}\left\{1-\exp \left[-2 \chi_{\text {soft }}(s, \vec{b})-2 \chi_{\text {hard }}(s, \vec{b})\right]\right\}, \\
& \sigma_{\text {tot }}=2 \int \mathrm{d}^{2} \vec{b}\left\{1-\exp \left[-\chi_{\text {soft }}(s, \vec{b})-\chi_{\text {hard }}(s, \vec{b})\right]\right\},
\end{aligned}
$$


where the scattering is compounded as a sum of QCD ladders via hard and soft processes through the eikonals $\chi_{\text {hard }}$ and $\chi_{\text {soft }}$. It should be noted that we have ignored spin-dependent effects and the small real part of the scattering amplitude, both good approximations at high energies (see e.g. [75]). Now, if the eikonal function, $\chi(s, \vec{b}) \equiv \chi_{\text {soft }}(s, \vec{b})+\chi_{\text {hard }}(s, \vec{b})=\lambda / 2$, indicates the mean number of partonic interaction pairs at impact parameter $\vec{b}$, the probability $p_{n}$ for having $n$ independent partonic interactions using Poisson statistics reads, $p_{n}=\left(\lambda^{n} / n !\right) \mathrm{e}^{-\lambda 3}$. Therefore, the factor $1-\mathrm{e}^{-2 \chi}=\sum_{n=1}^{\infty} p_{n}$ in Eq. (8) can be interpreted semiclassically as the probability that at least 1 of the 2 protons is broken up in a collision at impact parameter $\vec{b}$. With this in mind, the inelastic cross section is simply the integral over all collision impact parameters of the probability of having at least 1 interaction, yielding a mean minijet multiplicity of $\left\langle n_{\mathrm{jet}}\right\rangle \approx \sigma_{\mathrm{QCD}} / \sigma_{\text {inel }}[78]$. The leading contenders to approximate the (unknown) cross sections at cosmic ray energies, SIBYLL [79] and QGSJET [80], share the eikonal approximation but differ in their ansätse for the eikonals. In both cases, the core of dominant scattering at very high energies is the parton-parton minijet cross section given in Eq. (1),

$$
\chi_{\text {hard }}=\frac{1}{2} \sigma_{\mathrm{QCD}}\left(s, p_{T}^{\text {cutoff }}\right) A(s, \vec{b}),
$$

where the normalized profile function, $\int \mathrm{d}^{2} \vec{b} A(s, \vec{b})=1$, indicates the distribution of partons in the plane transverse to the collision axis.

In the QGSJET-like models, the core of the hard eikonal is dressed with a soft-pomeron pre-evolution factor. This amounts to taking a parton distribution which is Gaussian in the transverse coordinate distance $|\vec{b}|$,

$$
A(s, \vec{b})=\frac{\mathrm{e}^{-|\vec{b}|^{2} / R^{2}(s)}}{\pi R^{2}(s)},
$$

where $R^{2}(s) \sim 4 R_{0}^{2}+4 \alpha_{\text {eff }}^{\prime} \ln ^{2}\left(s / s_{0}\right)$, with $\alpha_{\text {eff }}^{\prime} \approx 0.11 \mathrm{GeV}^{-2}$. Fits to collider data have been carried out [81] using a Gaussian profile function with energy-independent width, $R_{0}$, which was allowed to vary in the fit. Under the assumption that the partons contributing to jet production are uniformly distributed in the transverse space all over the proton, one can obtain a reasonable fit to the data with $R_{0}=3.5 \mathrm{GeV}^{-2}$ and $p_{T}^{\text {cutoff }}=3.5 \mathrm{GeV}$. However, if one allows for the possibility of parton clustering, $R_{0}$ shrinks to $1.5 \mathrm{GeV}^{-2}$ with $p_{T}^{\text {cutoff }}=2.5 \mathrm{GeV}$. This leads to a smaller rise of the cross section with energy. In fact, the CDF Collaboration has reported [82] measurements which may indicate that partons are distributed in clusters inside the proton. Specifically, measurements of the 4-jet to 2-jet ratio for a jet transverse energy cutoff of $5 \mathrm{GeV}$ when conveniently express in term of the effective cross section [83] lead to

$$
\sigma_{\text {eff }}=\frac{1}{2} \frac{\left[\sigma_{2-\text { jet }}\right]^{2}}{\sigma_{4-\text { jet }}}=14.5 \pm 1.7_{-2.3}^{+1.7} \mathrm{mb} .
$$

Within the eikonal unitarization, this corresponds to $\sigma_{\text {eff }}=8 \pi R_{0}^{2}$. From Eq. (12), $R_{0} \approx 1.5 \mathrm{GeV}^{-2}$, which is consistent with the clustering hypothesis.

\footnotetext{
${ }^{3}$ This relation can be derived within a field theoretical context [76]. using the Abramovski-GribovKancheli (AGK) cutting rules [77].
} 
In SIBYLL-like models, the transverse density distribution is taken as the Fourier transform of the proton electric form factor, resulting in an energy-independent exponential (rather than Gaussian) fall-off of the parton density profile with $|\vec{b}|$. The main characteristics of the $p p$ cascade spectrum resulting from these choices are readily predictable: the harder form of the SIBYLL form factor allows a greater retention of energy by the leading particle, and hence less available for the ensuing shower. Consequently, on average SIBYLL-like models predict a smaller multiplicity than QGSJET-like models (see e.g. [84-87]).

At high energy, $\chi_{\text {soft }} \ll \chi_{\text {hard }}$, and so the inelastic cross section is dominated by the hard eikonal. For impact parameters larger than some threshold, $b_{s}$, where $\chi_{\text {hard }}\left(s, b_{s}\right) \gg 1$, the damping from the exponential term in the Gaussian profile function of Eq. (11) is so strong that any increase in $\sigma_{\mathrm{QCD}}$ does not significantly alter the contribution to the inelastic cross section from the region where $|\vec{b}|<b_{s}$. At high energy, with the appropriate choice of normalization, the cross section in Eq. (6) can be well-approximated by a power law. Hence, by taking $\sigma_{\mathrm{QCD}} \sim s^{\Delta_{H}}$, one fixes $b_{s}^{2} \sim 4 \alpha_{\text {eff }}^{\prime} \Delta_{\mathrm{H}} \ln ^{2}\left(s / s_{0}\right)$ [86]. This implies that the growth of the inelastic cross section according to QGSJET-like models is given by

$$
\begin{aligned}
\sigma_{\text {inel }} & \sim \int \mathrm{d}^{2} \vec{b} \Theta\left(b_{s}-|\vec{b}|\right)=\pi b_{s}^{2} \sim 4 \pi \alpha_{\text {eff }}^{\prime} \Delta_{\mathrm{H}} \ln ^{2}\left(s / s_{0}\right) \\
& \sim 0.52 \Delta_{\mathrm{H}} \ln ^{2}\left(s / s_{0}\right) \mathrm{mb} .
\end{aligned}
$$

For SIBYLL-like models, where Eq. (11) is replaced by the Fourier transform of the proton electric form factor, the growth of the inelastic cross section also saturates the $\ln ^{2} s$ Froissart bound [88], but with a multiplicative constant which is larger than the one in QGSJET-like models [86]. Namely,

$$
\sigma_{\text {inel }} \sim 3.2 \Delta_{\mathrm{H}}^{2} \ln ^{2}\left(s / s_{0}\right) \mathrm{mb} .
$$

Fig. 5 illustrates the large range of predictions for $p p$ inelastic cross section which remain consistent with HERA data. When the two leading order approximations discussed above are extrapolated to higher energies, both are consistent with existing cosmic ray data. Note, however, that in both cases the range of allowed cross sections at high energy varies by a factor of about 2-3. A point worth noting at this juncture: a number of approaches have been used to extract the $p p$ cross section from cosmic ray shower data [93-96]. The points in Fig. 5 correspond to the most up-todate estimate [90].

There are three event generators, SIBYLL [79], QGSJET [80], and DPMJET [97] which are tailored specifically for simulation of hadronic interactions up to the highest cosmic ray energies. ${ }^{4}$ The latest versions of these packages are SIBYLL 2.1 [99], QGSJET 01 [100], and DPMJET III [101]; respectively. In QGSJET, both the soft and hard processes are formulated in terms of Pomeron exchanges. To describe the minijets, the soft Pomeron mutates into a "semihard Pomeron," an ordinary soft Pomeron with the middle piece replaced by a QCD parton ladder, as sketched in the

\footnotetext{
${ }^{4}$ Additionally, a new event generator, neXus [98], is available for simulation of the region below $\sqrt{s} \sim 10^{4} \mathrm{GeV}$.
} 


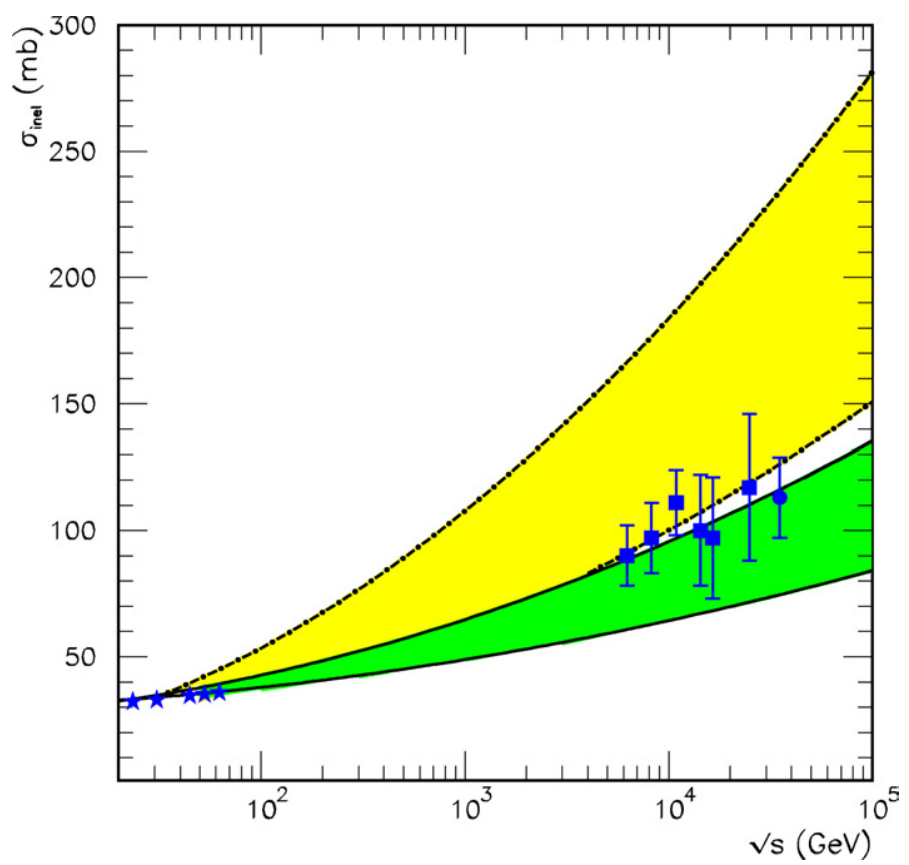

Fig. 5. Energy dependence of the $p p$ inelastic cross section as predicted by Eqs. (13) and (14) with $0.3<\Delta_{\mathrm{H}}<0.4$. The darkly shaded region between the solid lines corresponds to the model with Gaussian parton distribution in $\vec{b}$. The region between the dashed-dotted lines corresponds to the model with exponential fall-off of the parton density in $\vec{b}$. In both cases the cross sections are normalized to reproduce the data $(\star)$ [89] from the CERN Intersecting Storage Ring (ISR) at $30 \mathrm{GeV}$. Also shown are estimates [90] of the inelastic $p p$ cross section as derived from measurements of the inelastic $p$-air cross section by the

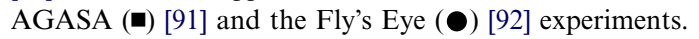

previous paragraph. This is generally referred to as the "quasi-eikonal" model. In contrast, SIBYLL and DPMJET follow a "two channel" eikonal model, where the soft and the semi-hard regimes are demarcated by a sharp cut in the transverse momentum: SIBYLL 2.1 uses a cutoff parametrization inspired in the double leading logarithmic approximation of the DGLAP equations

$$
p_{T}^{\text {cutoff }}(\sqrt{s})=p_{T}^{0}+0.065 \mathrm{GeV} \exp [0.9 \sqrt{\ln s}],
$$

whereas DPMJET III uses an ad hoc parametrization for the transverse momentum cutoff

$$
p_{T}^{\text {cutoff }}(\sqrt{s})=p_{T}^{0}+0.12 \mathrm{GeV}\left[\log _{10}(\sqrt{s} / 50 \mathrm{GeV})\right]^{3},
$$

where $p_{T}^{0}=2.5 \mathrm{GeV}[68]$.

The transition process from asymptotically free partons to colour-neutral hadrons is described in all codes by string fragmentation models [102]. Different choices of fragmentation functions can lead to some differences in the hadron multiplicities. However, the main difference in the predictions of QGSJET-like and SIBYLL-like models arises from different assumptions in extrapolation of the parton distribution function to low energy. 


\subsection{Hadronic interactions in the Earth's atmosphere}

Now we turn to nucleus-nucleus interactions, which cause additional headaches for event generators which must somehow extrapolate $p p$ interactions to simulate the proton-air collisions of interest. All the event generators described above adopt the Glauber formalism [71], which is equivalent to the eikonal approximation in nucleon-nucleon scattering, except that the nucleon density functions of the target nucleus are folded with that of the nucleon. The inelastic and production cross sections read:

$$
\begin{aligned}
& \tilde{\sigma}_{\text {inel }} \approx \int \mathrm{d}^{2} \vec{b}\left\{1-\exp \left[\sigma_{\text {tot }} T_{A}(\vec{b})\right]\right\}, \\
& \widetilde{\sigma}_{\text {prod }} \approx \int \mathrm{d}^{2} \vec{b}\left\{1-\exp \left[\sigma_{\text {inel }} T_{A}(\vec{b})\right]\right\},
\end{aligned}
$$

where $T_{A}(\vec{b})$ is the transverse density of hadronic matter of the target nucleus folded with that of the projectile hadron. Here, $\sigma_{\text {inel }}$ and $\sigma_{\text {tot }}$ are given by Eqs. (8) and (9), respectively. The $p$-air inelastic cross section is the sum of the "quasi-elastic" cross section, which corresponds to cases where the target nucleus breaks up without production of any new particles, and the production cross section, in which at least one new particle is generated. Clearly the development of EAS is mainly sensitive to the production cross section. Overall, the geometrically large size of nitrogen and oxygen nuclei dominates the inclusive proton-target cross section, and as a result the disagreement from model-dependent extrapolation is not more than about $15 \%$.

The event generators also make different choices in their handling of nucleus-air collisions [103,104]. Models of nucleus-nucleus interactions are particularly important to describe the first few generations of secondaries in cosmic ray showers produced by nuclei. Measurements of proton-nucleus reactions at lower energies [105] suggested that the charged multiplicity from a soft production mechanism should simply scale with the number of nucleons that participate in the collision [106], thus allowing for comparison of different nuclear systems based on simple nucleon-nucleon superposition models. The particle densities are sensitive to the relative contributions of soft and hard processes [107,108]. More recent experimental input suggests a simple superposition model is not completely realistic. Specifically, $\mathrm{RHIC}^{5}$ data have shown that the observed central particle densities [109-111] are smaller than predictions from conventional multi-string models, with differences of $20-30 \%[112,113]$. To reduce the multiplicity in the models, the percolation process, which leads with increasing density to more and more fusion strings, has been proposed [114-116]. Very recently, the data from $\mathrm{d}-\mathrm{Au}$ collisions collected by the PHOBOS Collaboration [117] were used to improve the event generator DPMJET III and bring the predicted multiplicity in line with the data [118].

So far the discussion has concerned $p$-air and nucleus-air interactions. Of course in air shower simulations, we are also concerned with $\pi$-air interactions. Each

\footnotetext{
${ }^{5}$ The relativistic heavy ion collider (RHIC) collides ultra relativistic ions at energies up to $0.2 \mathrm{TeV} / \mathrm{N}$. RHIC has two large detectors, STAR and PHENIX and two smaller experiments: BRAHMS and PHOBOS.
} 

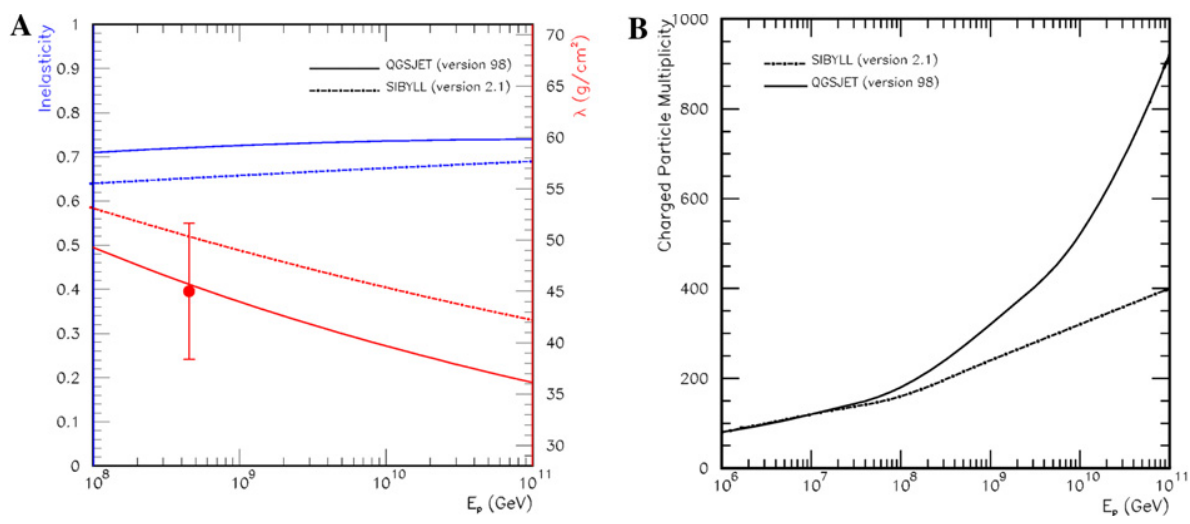

Fig. 6. (A) The slowly rising curves indicate the mean inelasticity in proton-air collisions as predicted by QGSJET and SIBYLL. The falling curves indicate the proton mean free path in the atmosphere. The data point is from Fly's Eye measurements [92]. (B) Mean multiplicity of charged secondary particles produced in inelastic proton-air collisions processed with QGSJET and SIBYLL.

approach discussed above handles $\pi p$ collisions using the same interaction model it uses for $p p$ collisions. For energies of interest, both models predict, on average, a $\pi p$ inelastic cross section about $20 \%$ smaller than the $p p$ cross section [86].

Since the codes described above are still being refined, the disparity between them can vary even from version to version. At the end of the day, however, the relevant parameters boil down to two: the mean free path, $\lambda=\left(\widetilde{\sigma}_{\text {prod }} n\right)^{-1}$, and the inelasticity, $K=1-E_{\text {lead }} / E_{\text {proj, }}$, where $n$ is the number density of atmospheric target nucleons, $E_{\text {lead }}$ is the energy of the most energetic hadron with a long lifetime, and $E_{\text {proj }}$ is the energy of the projectile particle. The first parameter characterizes the frequency of interactions, whereas the second one quantifies the energy lost per collision. Overall, SIBYLL has a shorter mean free path and a smaller inelasticity than QGSJET, as indicated in Fig. 6. Since a shorter mean free path tends to compensate a smaller inelasticity, the two codes generate similar predictions for an air shower which has lived through several generations. The different predictions for the mean charged particle multiplicity in proton-air collisions are shown in Fig. 6. Both models predict the same multiplicity below about $10^{7} \mathrm{GeV}$, but the predictions diverge above that energy. Such a divergence readily increases with rising energy. While QGSJET predicts a power law-like increase of the number of secondaries up to the highest energy, SIBYLL multiplicity exhibits a logarithmic growth. As it is extremely difficult to observe the first interactions experimentally, it is not straightforward to determine which model is closer to reality. In Section 3, however, we will discuss observables which may offer a hint of which model better predicts overall shower characteristics.

\subsection{Measurements of forward processes at the LHC}

Interpretation of cosmic ray data suffers from the lack of knowledge of high energy hadronic interaction models. Hard interactions with high momentum transfer 
are calculable in perturbation theory using QCD. At present, collider experiments have mostly concentrated on these hard processes in the central region, thereby excluding soft processes in the far-forward direction. These low momentum transfer processes, which are of great interest in the development of cosmic ray EAS, are not calculable from the fundamental QCD Lagrangian.

Some guidance towards understanding hadronic processes in the forward direction may come directly from measurements of hadrons in airshowers [119]. However, the most useful experimental input in the foreseeable future will likely come from the LHC. This machine, expected to become operational in 2007 or so, will provide $p p$ collisions with $\sqrt{s}=14 \mathrm{TeV}$ and luminosity up to $L \approx 10^{34} \mathrm{~cm}^{-2} \mathrm{~s}^{-1}$ [120], as well as, a few years later, lead-lead ion collisions with $\sqrt{s}=1000 \mathrm{TeV}$. Two general-purpose experiments, ATLAS and CMS, presently cover up to $|\eta|<5$. A dedicated heavy ion detector, ALICE, will also operate at this collider. ${ }^{6}$

The interesting low momentum transfer processes tend to populate the region at very small angles $\vartheta$ with respect to the beam direction. The distribution of pseudorapidity, $\eta=-\ln \tan (\vartheta / 2)$, and the energy flow distribution are shown in Fig. 7. While the particle multiplicity is greatest in the low $|\eta|$ region, it is clearly seen that the energy flow is peaked at small production angles (large $|\eta|$ ).

A study of diffraction, in $p p$ as well as heavy ion collisions, must use detectors with excellent forward acceptance to allow for a comparison with cosmic ray data. Dedicated runs of the LHC with lower luminosity $\left(L=10^{28} \mathrm{~cm}^{-2} \mathrm{~s}^{-1}\right)$ and specially tuned beam optics are planned to study these diffractive events. At present the only approved experiment at LHC with a capability of measuring, to some extent, very forward particles is TOTEM [122,121,123], which will comprise Roman pots placed on each side of the CMS interaction region and forward trackers which cover the pseudorapidity range $3.0 \leqslant \eta \leqslant 6.8$. It should be mentioned, however, that the fragmentation region that plays a crucial role in the development of EAS corresponds to pseudorapidity range $6 \leqslant|\eta| \leqslant 10$.

The main goal of TOTEM is the measurement of elastic and total cross sections with an expected precision of about $1 \%$, in a luminosity independent manner. To calculate the total cross section in terms of the number of elastic and inelastic events measured by TOTEM, we can resort to the well-known optical theorem

$$
\sigma_{\mathrm{tot}}=\frac{8 \pi}{\sqrt{s}} \mathfrak{I m}[f(0)]
$$

where $f(\vartheta)$ satisfies

$$
\frac{\mathrm{d} \sigma_{\mathrm{el}}}{\mathrm{d} t}=\frac{4 \pi}{s} \frac{\mathrm{d} \sigma_{\mathrm{el}}}{\mathrm{d} \Omega}=\frac{4 \pi}{s}|f(\vartheta)|^{2},
$$

with $\vartheta$ the angle of the scattered proton with respect to the beam direction. Squaring Eq. (19) we obtain

\footnotetext{
${ }^{6}$ A $b$-physics experiment, LHC- $b$, is also under construction at the LHC and will offer particle identification in the range $1.9<\eta<4.9$.
} 


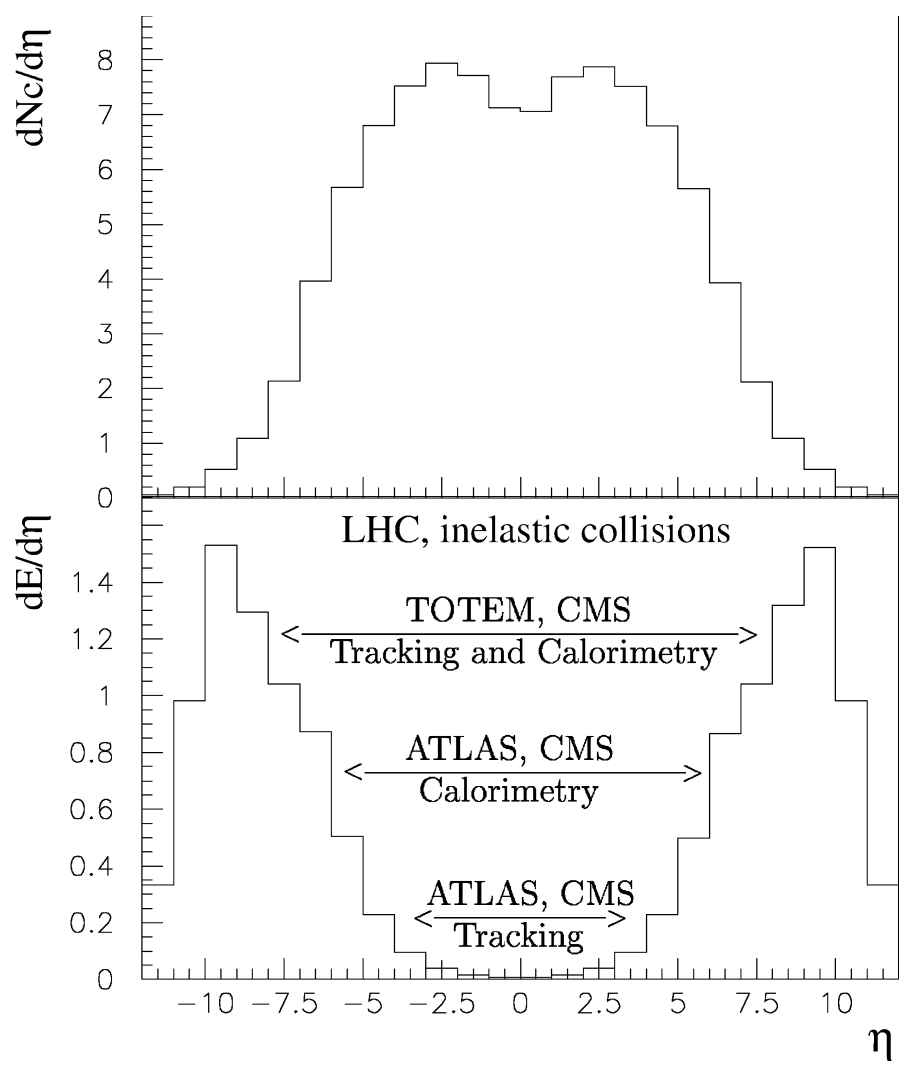

Fig. 7. Pseudorapidity distributions of charged particles (upper panel) and of the energy flow (lower panel) for $p p$ collisions at LHC [121].

$$
\sigma_{\text {tot }}^{2}=\left.\frac{16 \pi \mathfrak{I m}^{2}[f(0)]}{\mathfrak{R e}^{2}[f(0)]+\mathfrak{I m}^{2}[f(0)]} \frac{\mathrm{d} \sigma_{\mathrm{el}}}{\mathrm{d} t}\right|_{t=0}=\frac{16 \pi}{1+\rho^{2}} \frac{\left[\mathrm{d} N_{\mathrm{el}} / \mathrm{d} t\right]_{t=0}}{\mathscr{L}},
$$

where $\mathscr{L}$ is the integrated luminosity. Now, following [124,125], we can obtain the total cross section independently from $\mathscr{L}$, by using $\sigma_{\text {tot }}=\left(\sigma_{\text {el }}+\sigma_{\text {inel }}\right)=\left(N_{\text {el }}+N_{\text {inel }}\right) / \mathscr{L}$

$$
\sigma_{\mathrm{tot}}=\frac{16 \pi}{1+\rho^{2}} \frac{\left[\mathrm{d} N_{\mathrm{el}} / \mathrm{d} t\right]_{t=0}}{\left(N_{\mathrm{el}}+N_{\mathrm{inel}}\right)} .
$$

Here, $N_{\mathrm{el}}$ and $N_{\text {inel }}$ are the numbers of elastic and inelastic events, and $\rho=0.10 \pm 0.01$ is the ratio between the real and imaginary parts of the forward scattering amplitude $[126] .{ }^{7}$ The difficult aspect of this measurement is obtaining a good extrapolation of the cross section for low momentum transfer. Recall that $-t=s(1-\cos \vartheta) /$ $2 \simeq s \vartheta^{2} / 4$, and so $t \rightarrow 0$ implies a measurement in the extreme forward direction.

\footnotetext{
${ }^{7}$ Note that the quoted value of $\rho$ is an extrapolation to $\sqrt{s}=14 \mathrm{TeV}$, and may be measured by the LHC experiments. Otherwise, it will contribute to the uncertainty in $\sigma_{\text {tot }}$.
} 
The TOTEM experiment aims to measure down to values of $|t| \approx \times 10^{-3} \mathrm{GeV}^{2}$, which corresponds to $\vartheta \approx 4.5 \mu \mathrm{rad}$ [123]. The design for the pseudorapidity range $5.5<|\eta|<6.8$ is under discussion in a joint CMS/TOTEM working group. A tungsten Čerenkov calorimeter known as CASTOR has also been proposed which would compliment the measurements of TOTEM and CMS for $|\eta|<6.8$ and facilitate simultaneous measurements of particle flow in diffractive and non-diffractive events.

The ATLAS experiment is planning to implement additional detectors to cover the forward diffractive regions with tracking and/or calorimetry [127], with proposed coverage for the region $|t| \approx 6 \times 10^{-4} \mathrm{GeV}^{2}$.

In summary, existing event generators rely on theoretical extrapolations of existing data up to the energies near the GZK energy. There is a general consensus in the community that to understand the development of EAS at these extreme energies, new inputs from accelerator experiments are needed. A series of workshops have been organized to discuss what experimental inputs are most needed [128-130]; a preliminary list of the requirements includes [131]: (i) measurements of total and inelastic cross sections for $p p, p A, A A$ (ii) measurements of the ratio between soft diffractive and semi-hard processes, $\sigma_{\text {diff }} / \sigma_{\text {inel }}$, and (iii) measurement of inclusive final state hadrons in the two momentum ranges, $0.8<x<1.0$ and $0.1<x<0.8$.

\section{Electromagnetic processes}

In this section, we describe the electromagnetic interactions of relevance in ultra high energy shower development. The most important processes are electron and muon bremsstrahlung and pair production. We also discuss the Landau-Pomeranchuk-Migdal (LPM) effect, which suppresses the cross sections for pair production and bremsstrahlung above roughly $10^{10} \mathrm{GeV}$, and photon conversion in the geomagnetic field, which to a large degree compensates for the LPM effect in terms of shower observables. We comment further on shower observables such as the age parameter, Moliere radius, and shower size within the context of the Nishimura, Kamata, and Greisen (NKG) model. Finally, we discuss the extension of the NKG formalism to the corresponding shower parameters describing the lateral spread and the longitudinal development of EAS initiated by hadrons.

\subsection{The electromagnetic component}

The evolution of an extensive air shower is dominated by electromagnetic processes. The interaction of a baryonic cosmic ray with an air nucleus high in the atmosphere leads to a cascade of secondary mesons and nucleons. The first few generations of charged pions interact again, producing a hadronic core, which continues to feed the electromagnetic and muonic components of the showers. Up to about $50 \mathrm{~km}$ above sea level, the density of atmospheric target nucleons is $n \sim 10^{20} \mathrm{~cm}^{-3}$, and so even for relatively low energies, say $E_{\pi^{ \pm}} \approx 1 \mathrm{TeV}$, the probability of decay before interaction falls below $10 \%$. Ultimately, the electromagnetic cascade dissipates around $90 \%$ of the primary particle's energy, and hence the total 
number of electromagnetic particles is very nearly proportional to the shower energy [132].

By the time a vertically incident $10^{11} \mathrm{GeV}$ proton shower reaches the ground, there are about $10^{11}$ secondaries with energy above $90 \mathrm{keV}$ in the the annular region extending $8 \mathrm{~m}-8 \mathrm{~km}$ from the shower core. Of these, $99 \%$ are photons, electrons, and positrons, with a typical ratio of $\gamma$ to $e^{+} e^{-}$of 9 to 1 . Their mean energy is around $10 \mathrm{MeV}$ and they transport $85 \%$ of the total energy at ground level. Of course, photon-induced showers are even more dominated by the electromagnetic channel, as the only significant muon generation mechanism in this case is the decay of charged pions and kaons produced in $\gamma$-air interactions [133].

It is worth mentioning that these figures dramatically change for the case of very inclined showers. For a primary zenith angle, $\theta>70^{\circ}$, the electromagnetic component becomes attenuated exponentially with atmospheric depth, being almost completely absorbed at ground level. We remind the reader that the vertical atmosphere is $\approx 1000 \mathrm{~g} / \mathrm{cm}^{2}$, and is about 36 times deeper for completely horizontal showers (see Fig. 2). As a result, most of the energy at ground level from an inclined shower is carried by muons.

In contrast to hadronic collisions, the electromagnetic interactions of shower particles can be calculated very accurately from quantum electrodynamics. Electromagnetic interactions are thus not a major source of systematic errors in shower simulations. The first comprehensive treatment of electromagnetic showers was elaborated by Rossi and Greissen [134]. This treatment was recently cast in a more pedagogical form by Gaisser [135], which we summarize in the subsequent paragraphs.

The generation of the electromagnetic component is driven by electron bremsstrahlung and pair production [136]. Eventually the average energy per particle drops below a critical energy, $\epsilon_{0}$, at which point ionization takes over from bremsstrahlung and pair production as the dominant energy loss mechanism. The $e^{ \pm}$energy loss rate due to bremsstrahlung radiation is nearly proportional to their energy, whereas the ionization loss rate varies only logarithmically with the $e^{ \pm}$energy. Though several different definitions of the critical energy appear in the literature [137], throughout this review we take the critical energy to be that at which the ionization loss per radiation length is equal to the electron energy, yielding $\epsilon_{0}=710 \mathrm{MeV} /\left(Z_{\text {eff }}+0.92\right) \sim 86 \mathrm{MeV}[138]^{8}{ }^{8}$ The changeover from radiation losses to ionization losses depopulates the shower. One can thus categorize the shower development in three phases: the growth phase, in which all the particles have energy $>\epsilon_{0}$; the shower maximum, $X_{\max }$; and the shower tail, where the particles only lose energy, get absorbed or decay.

The relevant quantities participating in the development of the electromagnetic cascade are the probability for an electron of energy $E$ to radiate a photon of energy $k=y E$ and the probability for a photon to produce a pair $e^{+} e^{-}$in which one of the particles (hereafter $e^{-}$) has energy $E=x k$. These probabilities are determined by the properties of the air and the cross sections of the two processes.

\footnotetext{
${ }^{8}$ For altitudes up to $90 \mathrm{~km}$ above sea level, the air is a mixture of $78.09 \%$ of $\mathrm{N}_{2}, 20.95 \%$ of $\mathrm{O}_{2}$, and $0.96 \%$ of other gases [139]. Such a mixture is generally modeled as an homogeneous substance with atomic charge and mass numbers $Z_{\text {eff }}=7.3$ and $A_{\text {eff }}=14.6$, respectively.
} 
In the energy range of interest, the impact parameter of the electron or photon is larger than an atomic radius, so the nuclear field is screened by its electron cloud. In the case of complete screening, where the momentum transfer is small, the cross section for bremsstrahlung can be approximated by [140]

$$
\frac{\mathrm{d} \sigma_{e \rightarrow \gamma}}{\mathrm{d} k} \approx \frac{A_{\mathrm{eff}}}{X_{0} N_{A} k}\left(\frac{4}{3}-\frac{4}{3} y+y^{2}\right),
$$

where $A_{\text {eff }}$ is the effective mass number of the air, $X_{0}$ is a constant, and $N_{A}$ is Avogadro's number. In the infrared limit (i.e., $y \ll 1$ ) this approximation is inaccurate at the level of about $2.5 \%$, which is small compared to typical experimental errors associated with cosmic air shower detectors. Of course, the approximation fails as $y \rightarrow 1$, when nuclear screening becomes incomplete, and as $y \rightarrow 0$, at which point the LPM and dielectric suppression effects become important, as we discuss below.

Using similar approximations, the cross section for pair production can be written as [140]

$$
\frac{\mathrm{d} \sigma_{\gamma \rightarrow e^{+} e^{-}}}{\mathrm{d} E} \approx \frac{A_{\mathrm{eff}}}{X_{0} N_{A}}\left(1-\frac{4}{3} x+\frac{4}{3} x^{2}\right) .
$$

The similarities between this expression and Eq. (23) are to be expected, as the Feynman diagrams for pair production and bremsstrahlung are variants of one another.

The probability for an electron to radiate a photon with energy in the range $(k, k+\mathrm{d} k)$ in traversing $\mathrm{d} t=\mathrm{d} X / X_{0}$ of atmosphere is

$$
\frac{\mathrm{d} \sigma_{e \rightarrow \gamma}}{\mathrm{d} k} \frac{X_{0} N_{A}}{A_{\text {eff }}} \mathrm{d} k \mathrm{~d} t \approx\left(y+\frac{4}{3} \frac{1-y}{y}\right) \mathrm{d} y \mathrm{~d} t
$$

whereas the corresponding probability density for a photon producing a pair, with electron energy in the range $(E, E+\mathrm{d} E)$, is

$$
\frac{\mathrm{d} \sigma_{\gamma \rightarrow e^{+} e^{-}}}{\mathrm{d} E} \frac{X_{0} N_{A}}{A_{\text {eff }}} \mathrm{d} E \mathrm{~d} t \approx\left(1-\frac{4}{3} x+\frac{4}{3} x^{2}\right) \mathrm{d} x \mathrm{~d} t
$$

The total probability for pair production per unit of $X_{0}$ follows from integration of Eq. (26),

$$
\int \frac{\mathrm{d} \sigma_{\gamma \rightarrow e^{+} e^{-}}}{\mathrm{d} E} \frac{X_{0} N_{A}}{A_{\mathrm{eff}}} \mathrm{d} E \approx \int_{0}^{1}\left(1-\frac{4}{3} x+\frac{4}{3} x^{2}\right) \mathrm{d} x=\frac{7}{9} .
$$

As can be seen from Eq. (25), the total probability for bremsstrahlung radiation is logarithmically divergent. However, this infrared divergence is eliminated by the interference of bremsstrahlung amplitudes from multiple scattering centers. This collective effect of the electric potential of several atoms is known as the Landau-Pomeranchuk-Migdal (LPM) effect [141,142]. Of course, the LPM suppression of the cross section results in an effective increase of the mean free path of electrons and photons. This effectively retards the development of the electromagnetic component of the shower. It is natural to introduce an energy scale, $E_{\mathrm{LPM}}$, at which the inelasticity is low enough that the LPM effect becomes significant [143]. Below $E_{\mathrm{LPM}}$, the energy loss rate due to bremsstrahlung is roughly 


$$
\frac{\mathrm{d} E}{\mathrm{~d} X} \approx-\frac{1}{X_{0}} \int_{0}^{1} y E\left(y+\frac{4}{3} \frac{1-y}{y}\right) \mathrm{d} y=-\frac{E}{X_{0}} .
$$

With this in mind, we now identify the constant $X_{0} \approx 36.7 \mathrm{~g} \mathrm{~cm}^{-2}$ with the radiation length in air, defined as the mean distance over which a high-energy electron loses 1/e of its energy, or equivalently $7 / 9$ of the mean free path for pair production by a high-energy photon [137].

The experimental confirmation of the LPM effect at Stanford Linear Accelerator Center (SLAC) [144,145] has motivated new analyses of its consequences in cosmic ray physics [146-150]. The most evident signatures of the LPM effect on shower development are a shift in the position of the shower maximum $X_{\max }$ and larger fluctuations in the shower development.

When considering the LPM effect in the development of air showers produced by ultra high energy cosmic rays, one has to keep in mind that the suppression in the cross sections is strongly dependent on the atmospheric depth. ${ }^{9}$ Since the upper atmosphere is very thin the LPM effect becomes noticeable only for photons and electrons with energies above $E_{\mathrm{LPM}} \sim 10^{10} \mathrm{GeV}$. For baryonic primaries the LPM effect does not become important until the primary energy exceeds $10^{12} \mathrm{GeV}$. This is because the electromagnetic shower does not commence until after a significant fraction of the primary energy has been dissipated through hadronic interactions. To give a visual impression of how the LPM effect slows down the initial growth of high energy photon-induced showers, we show the average longitudinal shower development of $10^{11} \mathrm{GeV}$ proton and $\gamma$-ray showers (generated using AIREs 2.6.0 [151]) with and without the LPM effect in Fig. 8.

At energies at which the LPM effect is important (viz., $E>E_{\mathrm{LPM}}$ ), $\gamma$-ray showers will have already commenced in the geomagnetic field at almost all latitudes. This reduces the energies of the primaries that reach the atmosphere, and thereby compensates the tendency of the LPM effect to retard the shower development. The first description of photon interactions in the geomagnetic field dates back at least as far as 1966 [152], with a punctuated revival of activity in the early 1980's [153]. More recently, a rekindling of interest in the topic has led to refined calculations [154-156]. Primary photons with energies above $10^{10} \mathrm{GeV}$ convert into $e^{+} e^{-}$pairs, which in turn emit synchrotron photons. Regardless of the primary energy, the spectrum of the resulting photon "preshower" entering the upper atmosphere extends over several decades below the primary photon energy, and is peaked at energies below $10^{10} \mathrm{GeV}$ [154]. The geomagnetic cooling thus switches on at about the same energy at which the LPM effect does, and thereby preempts the LPM-related observables which would otherwise be evident. Recent simulations [157] which include photon preshowering indicate that above $\sim 10^{11} \mathrm{GeV}$ this effect tends to accelerate the shower development, shifting the $X_{\max }$ to a smaller value than previous calculation suggested [158,159], and into a range consistent with $X_{\max }$ typical of a proton primary.

\footnotetext{
${ }^{9}$ The same occurs for dielectric suppression, although the influence is not as important as for the LPM effect [148].
} 


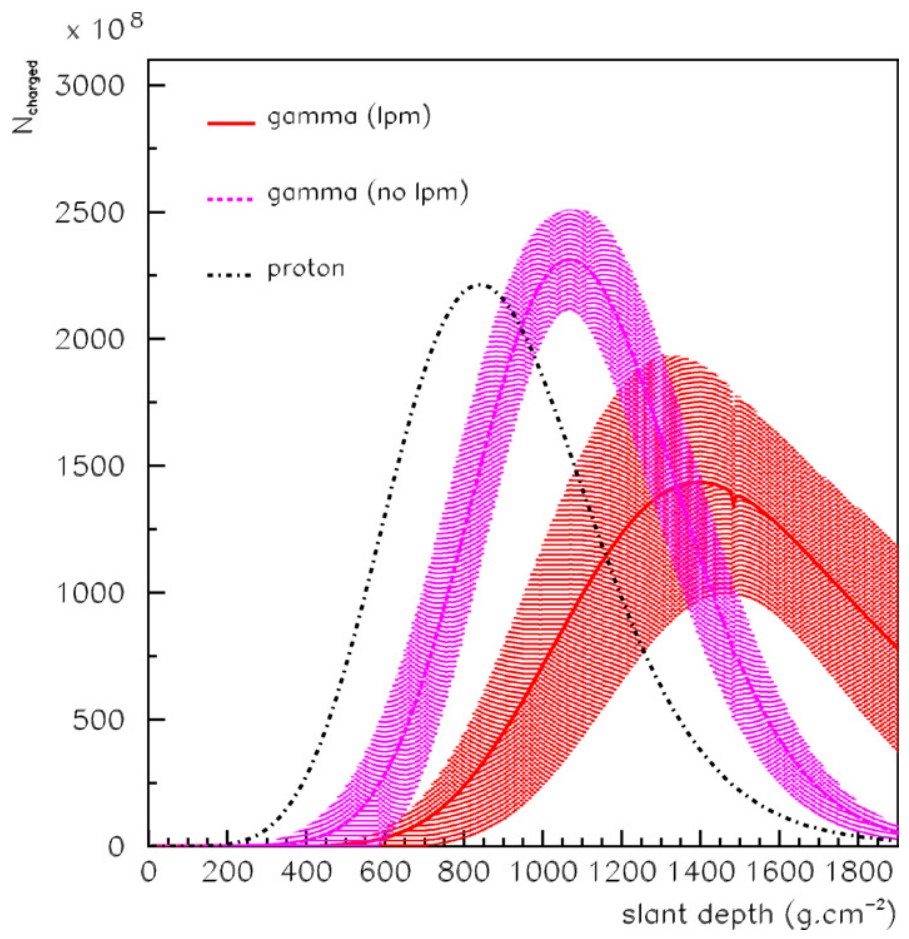

Fig. 8. Average longitudinal shower developments of $10^{11} \mathrm{GeV}$ proton (dashed-dotted line) and $\gamma$-rays with and without the LPM effect (solid and dotted lines, respectively). The primary zenith angle was set to $\theta=60^{\circ}$. The shadow area represents the intrinsic fluctuations of the showers. Larger fluctuations can be observed for $\gamma$-ray showers with the LPM effect, as expected.

The relevant parameter to determine both conversion probability and synchrotron emission is $E \times B_{\perp}$, where $E$ is the $\gamma$-ray energy and $B_{\perp}$ the transverse magnetic field. This leads to a large directional and geographical dependence of shower observables. Thus, each experiment has its own preferred direction for identifying primary $\gamma$ rays. For instance, Fig. 9 shows a map of the photon conversion probability in the geomagnetic field for all incident directions evaluated at the location of the HiRes experiment $\left(|\vec{B}|=0.53 \mathrm{G}, \imath=25^{\circ}\right.$, and $\left.\delta=14^{\circ}\right)[155]$. The smallest probabilities for conversion are found, not surprisingly, around the direction parallel to the local geomagnetic field. Note that this conversion-free region shrinks rapidly with increasing primary energy. A similar evaluation for the Southern Site of the Pierre Auger Observatory $\left(|\vec{B}|=0.25 \mathrm{G}, \imath=-35^{\circ}\right.$, and $\left.\delta=86^{\circ}\right)$ can be found in [154].

\subsubsection{Paper-and-pencil air shower modeling}

Most of the general features of an electromagnetic cascade can be understood in terms of the toy model due to Heitler [160]. In this model, the shower is imagined to develop exclusively via bremsstrahlung and pair production, each of which results in the conversion of one particle into two. As was previously discussed, these physical 

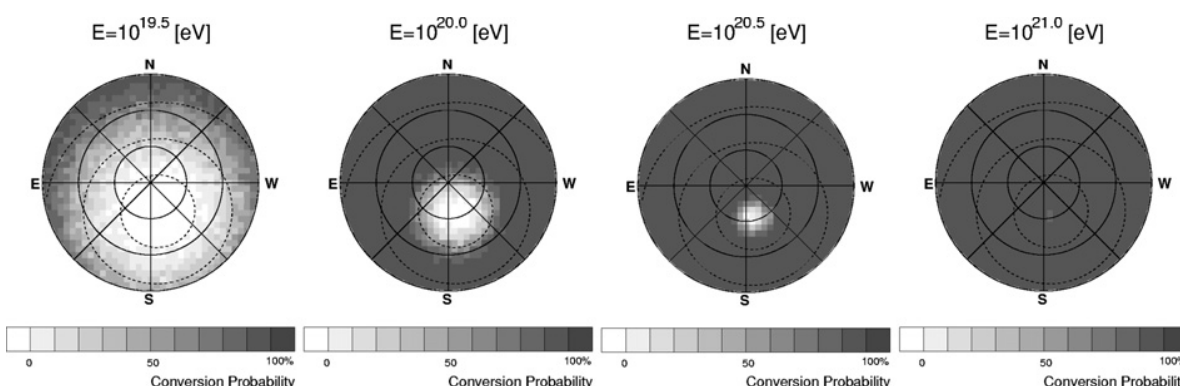

Conversion Probability
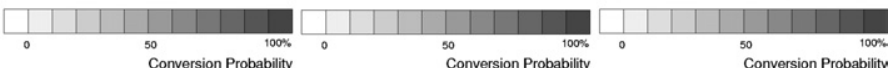

Fig. 9. Maps of $\gamma$-ray conversion probability in the geomagnetic field for several primary energies. Azimuths are as labeled, "N" denotes true north. The inner circles correspond to zenith angles $30^{\circ}, 60^{\circ}$, and horizon. Dashed curves indicate the opening angles of $30^{\circ}, 60^{\circ}$, and $90^{\circ}$ to the local magnetic field [155].

processes are characterized by an interaction length $X_{0}$. One can thus imagine the shower as a particle tree with branches that bifurcate every $X_{0}$, until they fall below a critical energy, $\epsilon_{0}$, at which point energy loss processes dominate. Up to $\epsilon_{0}$, the number of particles grows geometrically, so that after $n=X / X_{0}$ branchings, the total number of particles in the shower is $N \approx 2^{n}$. At the depth of shower maximum $X_{\max }$, all particles are at the critical energy, $\epsilon_{0}$, and the energy of the primary particle, $E_{0}$, is split among all the $N_{\max }=E_{0} / \epsilon_{0}$ particles. Putting this together, we get

$$
X_{\max } \approx X_{0} \frac{\ln \left(E_{0} / \epsilon_{0}\right)}{\ln 2} .
$$

In real life, the combination of the LPM and geomagnetic effects introduces large fluctuations in the value of $X_{\max }$ for photon showers. The prediction of this toy model roughly lies within the range of these fluctuations.

Even baryon-induced showers are dominated by electromagnetic processes, so this toy model is still enlightening for such cases. In particular, for proton showers, Eq. (29) tells us that the $X_{\max }$ scales logarithmically with primary energy, while $N_{\max }$ scales linearly. Moreover, to extend this discussion to heavy nuclei, we can apply the superposition principle as a reasonable first approximation. In this approximation, we pretend that the nucleus comprises unbound nucleons, such that the point of first interaction of one nucleon is independent of all the others. Specifically, a shower produced by a nucleus with energy $E_{A}$ and mass $A$ is modeled by a collection of $A$ proton showers, each with $A^{-1}$ of the nucleus energy. Modifying Eq. (29) accordingly one easily obtains $X_{\max } \propto \ln \left(E_{0} / A\right)$.

While the Heitler model is very useful for imparting a first intuition regarding global shower properties, the details of shower evolution are far too complex to be fully described by a simple analytical model. Full Monte Carlo simulation of interaction and transport of each individual particle is required for precise modeling of the shower development. At present two Monte Carlo packages are available to simulate EAS: CORSIKA (COsmic Ray SImulation for KAscade) [161] and AIRES (AIR shower Extended Simulation) [151]. Both programs provide fully four-dimensional simulations of the air showers initiated by protons, photons, and nuclei. To simulate 
hadronic physics the programs make use of the event generators described in Section 2. Propagation of particles takes into account the Earth's curvature and geomagnetic field. For further details on these codes the reader is referred to [162].

As a bridge between the first order approximation just described and a full-blown Monte Carlo treatment of air shower cascades, a hybrid method has recently been presented [163]. The approach is as follows. The first few interactions are treated using Monte Carlo event generators. The second step in the approach utilizes onedimensional cascade equations up to the point where the lateral spread of the particles becomes non-negligible, then the output of the cascade equations is treated again with Monte Carlo. The method shows a reasonable agreement when compared with results of the two detailed simulation packages [164].

\subsubsection{Electron lateral distribution function}

The transverse development of electromagnetic showers is dominated by Coulomb scattering of charged particles off the nuclei in the atmosphere. The lateral development in electromagnetic cascades in different materials scales well with the Molière radius $r_{\mathrm{M}}=E_{s} X_{0} / \epsilon_{0}$, which varies inversely with the density of the medium

$$
r_{\mathrm{M}}=r_{\mathrm{M}}\left(h_{\mathrm{OL}}\right) \frac{\rho_{\mathrm{atm}}\left(h_{\mathrm{OL}}\right)}{\rho_{\mathrm{atm}}(h)} \simeq \frac{9.0 \mathrm{~g} / \mathrm{cm}^{2}}{\rho_{\mathrm{atm}}(h)},
$$

where $E_{s} \approx 21 \mathrm{MeV}$ [137] and the subscript OL indicates a quantity taken at a given observation level.

Approximate calculations of cascade equations in three dimensions to derive the lateral structure function for a pure electromagnetic cascade in vertical showers were obtained by Nishimura and Kamata [165], and later worked out by Greisen [166] in the well-known NKG formula

$$
\rho(r)=\frac{N_{\mathrm{e}}}{r_{\mathrm{M}}^{2}} C\left(\frac{r}{r_{\mathrm{M}}}\right)^{s_{\mathrm{NKG}}-2}\left(1+\frac{r}{r_{\mathrm{M}}}\right)^{s_{\mathrm{NKG}}-4.5},
$$

where $N_{\mathrm{e}}$ is the total number of electrons, $r$ is the distance from the shower axis, and

$$
C=\frac{\Gamma\left(4.5-s_{\mathrm{NKG}}\right)}{2 \pi \Gamma\left(s_{\mathrm{NKG}}\right) \Gamma\left(4.5-2 s_{\mathrm{NKG}}\right)} .
$$

For a primary of energy $E_{0}$, the so-called "age parameter"

$$
s_{\mathrm{NKG}}=3\left[1+\frac{2 \ln \left(E_{0} / \epsilon_{0}\right)}{t}\right]^{-1},
$$

characterizes the stage of the shower development in terms of the depth of the shower in radiation lengths, i.e., $t=\int_{z}^{\infty} \rho_{\text {atm }}(z) \mathrm{d} z / X_{0}$.

The NKG formula may also be extended to describe showers initiated by baryons [167]. In such an extension, one finds a deviation of behavior of the Molière radius described in Eq. (30) when using a value of the age parameter which is derived from theoretical predictions for pure electromagnetic cascades. The need for a different age parameter to reproduce the electromagnetic component of hadronic induced showers has been addressed experimentally [168-175] and extensively studied by 
several authors [175-177]. It is possible to generalize the NKG formula for the electromagnetic component of baryon-induced showers by modifying the exponents in Eq. (31). From simulations, fits to lateral distribution functions (LDF) of electrons and positrons as a function of depth, $t$, yield an age parameter given by

$$
s=3\left(1+\frac{2 \beta}{t}\right)^{-1},
$$

where the floating parameter $\beta$ takes into account the above mentioned deviations from the theoretical value $s_{\mathrm{NKG}}$ [167].

The modified NKG formula provides a good description of the $e^{+} e^{-}$lateral distribution at all stages of shower development for values of $r$ sufficiently far from the hadronic core. Fortunately, this is the experimentally interesting region, since typical ground arrays can only measure densities at $r>100 \mathrm{~m}$ from the shower axis, where detectors are not saturated.

To illustrate the validity of this parametrization, we show in Fig. 10 (top) the Monte Carlo $e^{+} e^{-}$density distributions corresponding to a single $10^{10} \mathrm{GeV}$ proton shower at selected atmospheric depths. The total number of electrons obtained from the fit to each single shower is slightly lower than the true value due to the invalidity
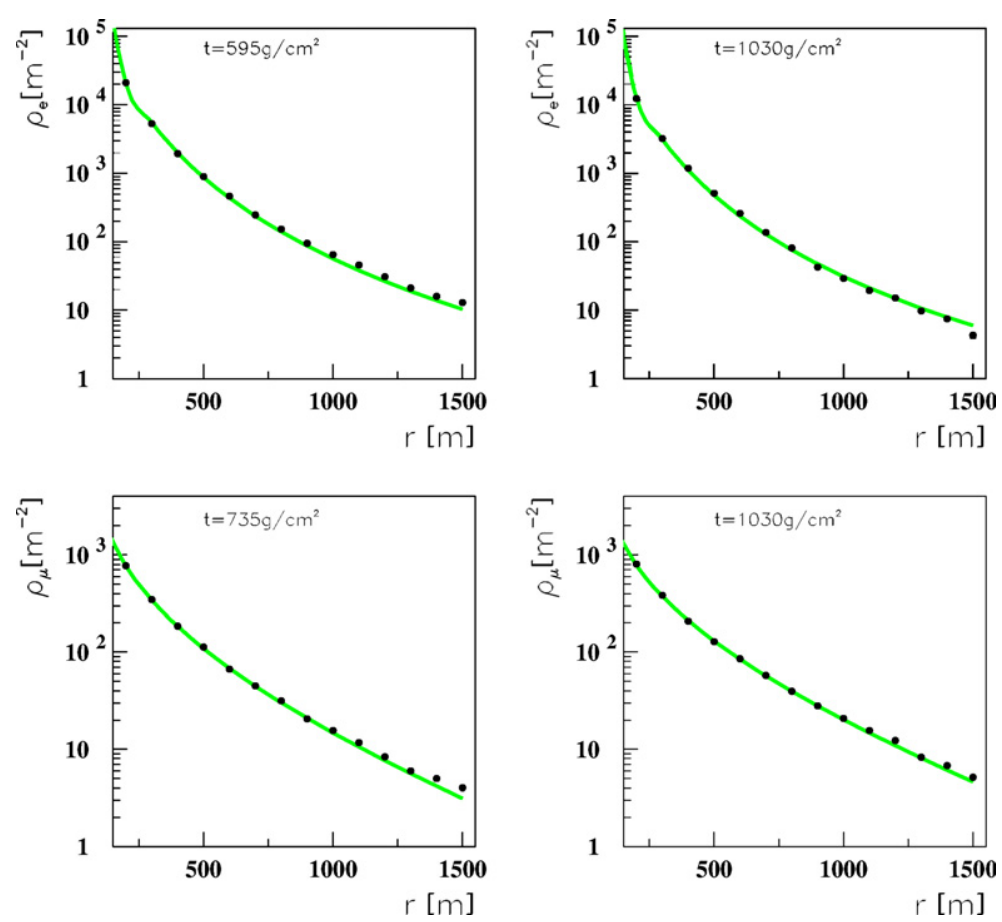

Fig. 10. Electron (top) and muon (bottom) lateral distributions of a $10^{10} \mathrm{GeV}$ vertical proton shower at different atmospheric altitudes. The solid lines are fits to the data using the NKG-like parametrization. The error bars are, in all cases, smaller than the points [167]. 
of the parametrization close to the shower core. It should be mentioned that an NKG-like formula can be used to parametrize the total particle's density observed in baryon-induced showers [178].

In the case of inclined showers, one normally analyzes particle densities in the plane perpendicular to the shower axis. Simply projecting distributions measured at the ground into this plane is a reasonable approach for near-vertical showers, but is not sufficient for inclined showers. In the latter case, additionally asymmetry is introduced because of both unequal attenuation of the electromagnetic components arriving at the ground earlier than and later than the core [167], and geometrical effects which also reduce the early compared to the late flux [179]. Moreover, deflections on the geomagnetic field become important for showers inclined by more than about $70^{\circ}$.

In the framework of cascade theory, any effect coming from the influence of the atmosphere should be accounted as a function of the slant depth $t$ [165]. Following this idea, a LDF valid at all zenith angles $\theta<70^{\circ}$ can be determined by considering

$$
t^{\prime}(\theta, \zeta)=t \sec \theta(1+\mathscr{K} \cos \zeta)^{-1},
$$

where $\zeta$ is the azimuthal angle in the shower plane, $\mathscr{K}=\mathscr{K}_{0} \tan \theta$, and $\mathscr{K}_{0}$ is a constant extracted from the fit $[167,180]$. Then, the particle lateral distributions for inclined showers $\rho\left(r, t^{\prime}\right)$ are given by the corresponding vertical LDF $\rho(r, t)$ but evaluated at slant depth $t^{\prime}(\theta, \zeta)$ where the dependence on the azimuthal angle is evident.

For zenith angles $\theta>70^{\circ}$, the surviving electromagnetic component at ground is mainly due to muon decay and, to a much smaller extent, hadronic interactions, pair production, and bremsstrahlung. As a result the lateral distribution follows that of the muon rather closely. In Fig. 11, the longitudinal development of the muon and electron components are shown. It is evident from the figure that for very inclined showers the electromagnetic development is due mostly to muon decay [182,181].

The consequences of the LPM effect and pair production in the geomagnetic field on the longitudinal cascade distribution initiated by photons were already discussed in this section. Since the lateral distribution of particles is strongly correlated with the development of the shower in the atmosphere, the LPM effect has consequences for the observed LDF at ground level. In particular, unconverted photons result in large fluctuations and steeper lateral profiles than nuclei [154].

In summary, the growth of the electromagnetic cascade is governed by bremsstrahlung and pair production. The mean free path for interactions via these processes depends on energy and atmospheric depth. Below $10^{10} \mathrm{GeV}$, each particle sees screened nuclei, while at higher energy collective effects suppress the cross section. On top of that, ultra high energy $\gamma$-ray interactions in the geomagnetic field also come into play, reducing the importance of the LPM cross section suppression.

The lateral distribution of the electromagnetic component of a shower can be effectively parametrized. The well-known NKG lateral distribution function, which strictly applies to only purely electromagnetic showers, can be extended to describe not only the electromagnetic portion of baryon-induced showers but also the signal produced by all particles reaching ground level. This provides a handle on one of the most useful shower observables available to surface arrays. 


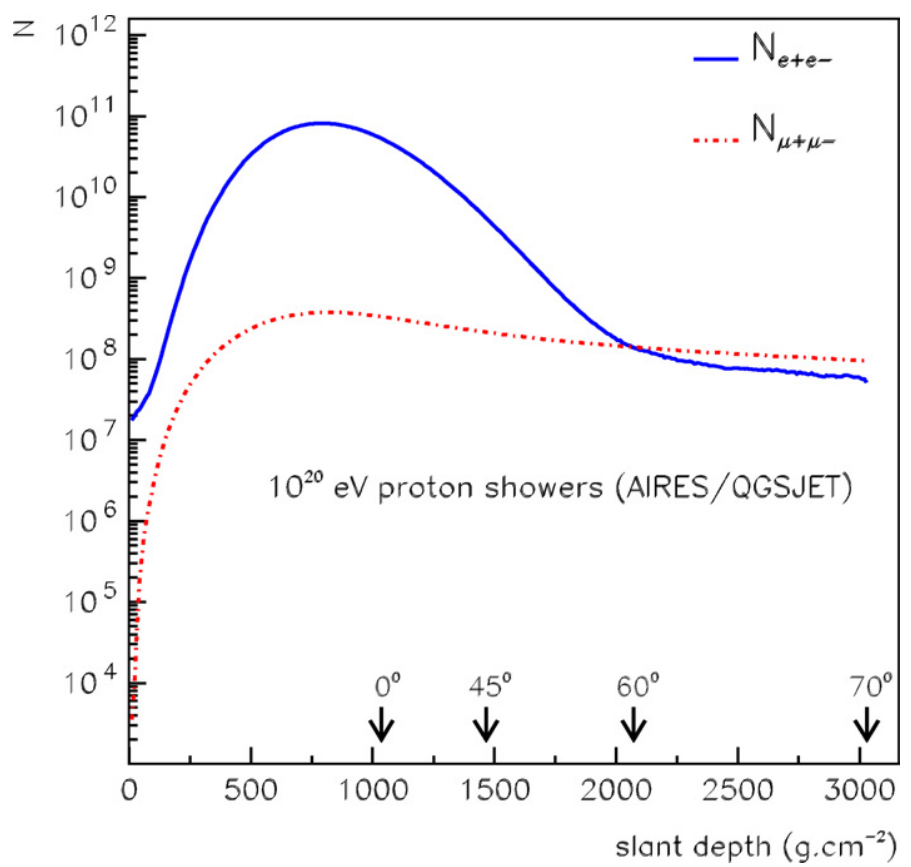

Fig. 11. Longitudinal development of muons and electrons as a function of the slant depth for $10^{11} \mathrm{GeV}$ proton-induced showers.

\subsection{The muon component}

The muonic component of EAS differs from the electromagnetic component for two main reasons. First, muons are generated through the decay of cooled $\left(E_{\pi^{ \pm}} \lesssim 1 \mathrm{TeV}\right)$ charged pions, and thus the muon content is sensitive to the initial baryonic content of the primary particle. Furthermore, since there is no "muonic cascade," the number of muons reaching the ground is much smaller than the number of electrons. Specifically, there are about $5 \times 10^{8}$ muons above $10 \mathrm{MeV}$ at ground level for a vertical $10^{11} \mathrm{GeV}$ proton induced shower. Second, the muon has a much smaller cross section for radiation and pair production than the electron, and so the muonic component of EAS develops differently than does the electromagnetic component. The smaller multiple scattering suffered by muons leads to earlier arrival times at the ground for muons than for the electromagnetic component.

The ratio of electrons to muons depends strongly on the distance from the core; for example, the $e^{+} e^{-}$to $\mu^{+} \mu^{-}$ratio for a $10^{11} \mathrm{GeV}$ vertical proton shower varies from 17 to 1 at $200 \mathrm{~m}$ from the core to 1 to 1 at $2000 \mathrm{~m}$. The ratio between the electromagnetic and muonic shower components behaves somewhat differently in the case of inclined showers. For zenith angles greater than $60^{\circ}$, the $e^{+} e^{-} / \mu^{+} \mu^{-}$ratio remains roughly constant at a given distance from the core. As the zenith angle grows beyond $60^{\circ}$, this ratio decreases, until at $\theta=75^{\circ}$, it is 400 times smaller than for a 
vertical shower. Another difference between inclined and vertical showers is that the average muon energy at ground changes dramatically. For horizontal showers, the lower energy muons are filtered out by a combination of energy loss mechanisms and the finite muon lifetime: for vertical showers, the average muon energy is $1 \mathrm{GeV}$, while for horizontal showers it is about two orders of magnitude greater. The muon densities obtained in shower simulations using SIBYLL 2.1 fall more rapidly with lateral distance to the shower core than those obtained using QGSJET 01 . This can be understood as a manifestation of the enhanced leading particle effect in SIBYLL, which can be traced to the relative hardness of the electromagnetic form factor profile function. The curvature of the distribution $\left(\mathrm{d}^{2} \rho_{\mu} / \mathrm{d} r^{2}\right)$ is measurably different in the two cases, and, with sufficient statistics, could possibly serve as a discriminator between hadronic interaction models, provided the primary species can be determined from some independent observable(s) [183].

High energy muons lose energy through $e^{+} e^{-}$pair production, muon-nucleus interaction, bremsstrahlung, and knock-on electron ( $\delta$-ray) production [184]. The first three processes are discrete in the sense that they are characterized by high inelasticity and a large mean free path. On the other hand, because of its short mean free path and its small inelasticity, knock-on electron production can be considered a continuous process. The muon bremsstrahlung cross section is suppressed by a factor of $\left(m_{e} / m_{\mu}\right)^{2}$ with respect to electron bremsstrahlung, see Eq. (23). Since the radiation length for air is about $36.7 \mathrm{~g} / \mathrm{cm}^{2}$, and the vertical atmospheric depth is $1000 \mathrm{~g} /$ $\mathrm{cm}^{2}$, muon bremsstrahlung is of negligible importance for vertical air shower development. Energy loss due to muon-nucleus interactions is somewhat smaller than muon bremsstrahlung. As can be seen in Fig. 12, energy loss by pair production is slightly more important than bremsstrahlung at about $1 \mathrm{GeV}$, and becomes increasingly dominant with energy. Finally, knock-on electrons have a very small mean free path (see Fig. 12), but also a very small inelasticity, so that this contribution to the energy loss is comparable to that from the hard processes.

In addition to muon production through charged pion decay, photons can directly generate muon pairs, or produce hadron pairs which in turn decay to muons. In the case of direct pair production, the large muon mass leads to a higher threshold for this process than for electron pair production. Furthermore, QED predicts that $\mu^{+} \mu^{-}$production is suppressed by a factor $\left(m_{e} / m_{\mu}\right)^{2}$ compared the Bethe-Heitler cross section. The cross section for hadron production by photons is much less certain, since it involves the hadronic structure of the photon. This has been measured at HERA for photon energies corresponding to $E_{\mathrm{lab}}=2 \times 10^{4} \mathrm{GeV}[185,186]$. This energy is still well below the energies of the highest energy cosmic rays, but nonetheless, these data do constrain the extrapolation of the cross sections to high energies. To give an idea of the rates, at $100 \mathrm{GeV}$ the cross section for $\gamma \rightarrow e^{+} e^{-}$is $\approx 650 \mathrm{mb}$, i.e., much larger than the cross sections for hadronic interaction $(\approx 1.4 \mathrm{mb})$ or for muon pair production $(\approx 0.015 \mathrm{mb})$.

\subsubsection{Muon lateral distribution function}

Now we consider the lateral distribution of the muon component of an extensive air shower. Unlike the electrons and photons, muons are relatively unaffected by 


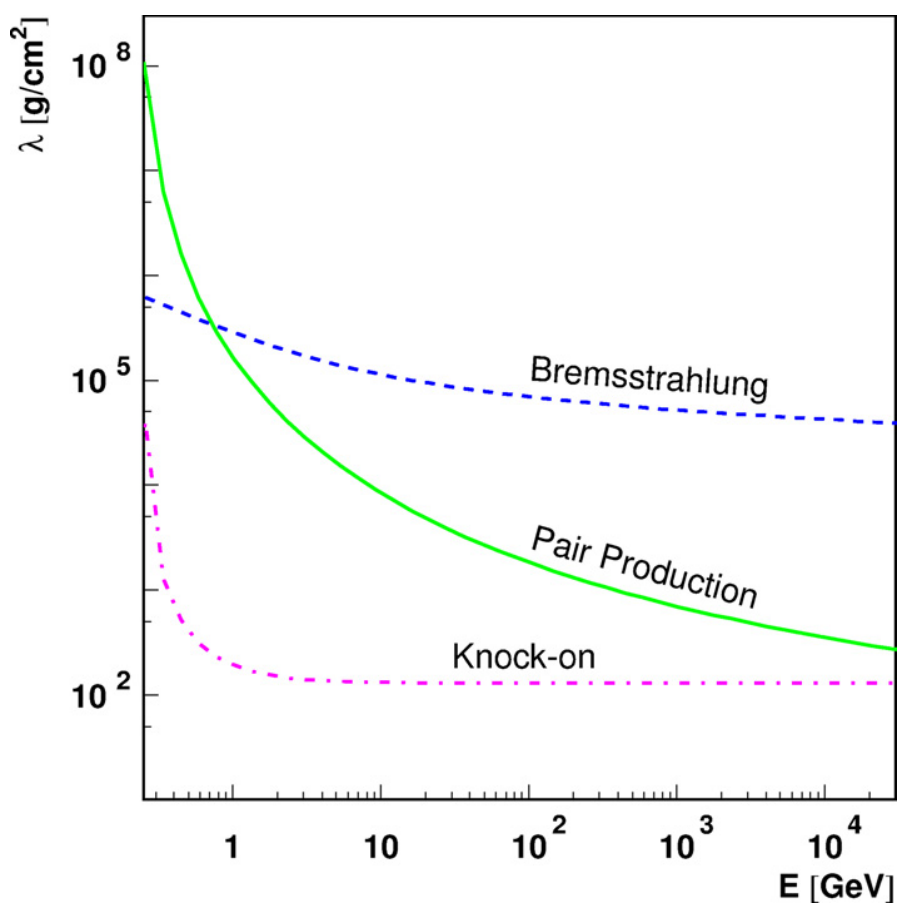

Fig. 12. Mean free path in air for the different muonic interactions as a function of the initial kinetic energy. This figure is courtesy of Sergio Sciutto.

multiple Coulomb scattering, and so their lateral distribution function (LDF) retains some information about the parent pion trajectories. In what follows, we first discuss a parameterization characterizing the muon LDF which is motivated only by the muon genealogy. After that, we add to the discussion the effect of the geomagnetic field on the evolution of the lateral distribution.

One of the earliest parameterizations of the muon LDF in vertical showers was empirically derived by Greisen [166]. This LDF was inspired by the NKG parametrization, and is factorized into two terms

$$
\rho_{\mu}(r)=N_{\mu}(t) f_{\mu}(r),
$$

where $N_{\mu}(t)$ gives the normalization as a function of depth $t$,

$$
f_{\mu}(r) \approx\left(\frac{r}{r_{\mathrm{G}}}\right)^{-0.75}\left(1+\frac{r}{r_{\mathrm{G}}}\right)^{-2.5}
$$

is a structure function describing the lateral shape of the shower, and $r_{\mathrm{G}}=320 \mathrm{~m}$ is analogous to the Molière radius.

Later, Vernov et al. [187] proposed a semi-analytical form of the structure function

$$
f_{\mu}(r) \approx\left(\frac{r}{r_{0}}\right)^{-\Gamma} \exp \left(-\frac{r}{r_{0}}\right)
$$


with $\Gamma=0.4$ and $r_{0}=80 \mathrm{~m}$. Similar approaches were also suggested by Hillas' group at the University of Leeds [188] and by the SUGAR Collaboration [189]. The slopes from Eqs. (37) and (38) are in very good agreement with each other at intermediate distance, but Eq. (38) predicts a distribution which is flatter close to the shower core and more strongly damped at large distances. These LDFs have been used to fit experimental data. However, neither function reproduces the whole radial range of an extensive air shower. This is a consequence of neglecting the shower age in formulating the structure functions. Very recently, the KASCADE Collaboration has used an NKG formula to fit muon density distributions [190]. The fits were performed close to the shower core $(r<200 \mathrm{~m})$ with non-conventional values of $r_{\mathrm{M}}$ and age parameter, $s$.

One expects there to be a dependence of the LDF parameters on the shower age. However, in contrast to electrons, muons in an air shower are less attenuated and little affected by Coulomb scattering, so the dependence of the LDF on the shower age is not the same as that exhibited by the electromagnetic component. The lateral growth of the shower is largely determined by the direction of emission of the parent particle and hence increases while the shower propagates downward. Two approaches for including shower age-dependence in the muon structure function have been discussed in the literature [191,167]. Here, we consider the more recent treatment, in which a Vernov-like approach is used taking a slope dependence on atmospheric depth, $\Gamma=2-s$, with $s$ as given in Eq. (34).

It is easily seen $[192,193]$ that, if the parent particles are created with a $p_{T}$ distribution, $p_{T} / p_{0} \exp \left(-p_{T} / p_{0}\right) \mathrm{d} p_{T} / p_{0}$, then the Vernov distribution at ground level has a value of $r_{0}$ given by

$$
r_{0}=\frac{2}{3}\left\langle h_{p}\right\rangle \frac{\left\langle p_{T}^{\mu}\right\rangle}{\left\langle E_{\mu}\right\rangle},
$$

where $\left\langle p_{T}\right\rangle=2 p_{0}$ is the mean transverse momentum, $\left\langle E_{\mu}\right\rangle$ the mean energy of muons, and $\left\langle h_{p}\right\rangle$ the mean height of muon production. These approximate expressions can serve to calculate the variation with depth of the parameters characterizing the lateral spread. The ratio $\left\langle p_{\perp}^{\mu}\right\rangle /\left\langle E_{\mu}\right\rangle$ can be considered constant while the shower develops [194] and the variation of $r_{0}$ with altitude is determined only by the dependence of $\left\langle h_{p}\right\rangle$ on depth, $t$.

Muons are produced in every pion generation and their energy distribution follows that of their parents. There are three phenomena contributing to the behavior of $\left\langle h_{p}\right\rangle$ as a function of $t$. The first is simply the dependence of the atmospheric density on height and temperature. For an isothermal atmosphere of scale $h_{0}$, one obtains $\left\langle h_{p}\right\rangle \propto h_{0} \ln \left(t / t_{p}\right)$. The second phenomenon is the "pionization" process: the competition between pion production and decay. The last contribution to the behavior of $\left\langle h_{p}\right\rangle$ is associated with systematics induced by hadronic interaction models. In what follows, we leave aside the issue of systematic errors and as an example adopt QGSJET 98 as the hadronic interaction model. Combining all these considerations, the characteristic radius $r_{0}(t)$ becomes

$$
r_{0}(t)=\frac{2}{3} \frac{\left\langle p_{T}^{\mu}\right\rangle}{\left\langle E_{\mu}\right\rangle} h_{0} \frac{t_{\mathrm{GL}}}{t} \ln \left[\frac{t}{t_{p}}\right],
$$


where the subscript GL indicates that a quantity is given at ground level. For $t=t_{\mathrm{GL}}$, Eq. (39) is recovered.

In Fig. 10, we show the $\mu^{+} \mu^{-}$density distributions for a single $10^{10} \mathrm{GeV}$ proton shower at various depths. Fits to the Vernov-like distribution are overlaid on the simulation results, indicating validity of the parametrization [167]. Furthermore, the total number of muons $N_{\mu}$ from the fits agrees quite well with the corresponding values predicted by the Monte Carlo simulation, even though the fits are performed at core distances $r>100 \mathrm{~m}$.

Muons can travel long distances without interacting with the medium, and consequently the ground density profiles are significantly modified by the Earth's magnetic field. The global shower observables, like longitudinal and lateral distributions, are not affected by the geomagnetic field for zenith angles $\theta<70^{\circ}$ [181]. However, for the case of very inclined showers, which are dominated by muons, the density at ground is rendered quite asymmetric by the geomagnetic field. In the remainder of this section we describe these effects quantitatively [194].

Consider a highly relativistic muon of energy $E_{\mu} \approx c p$ and transverse momentum $p_{T}$ that travels a distance $d$ to reach ground. This muon suffers a deviation $r$ from the shower axis given by

$$
r \simeq \frac{c p_{T} d}{E_{\mu}} .
$$

Now, it is easily seen that if the energy spectrum of muons is taken as $\phi(E)=A E_{\mu}^{-\gamma}$, the muon density is given by [194]

$$
\rho_{\mu}(r)=\frac{A}{2 \pi}\left(c p_{T} d\right)^{1-\gamma} r^{-3+\gamma} .
$$

To take into account the effect of the geomagnetic field, define a cartesian coordinate, $(x, y)$, in the plane transverse to the shower axis, with $y$ aligned to $\vec{B}_{\perp}$. The circular symmetry of the shower is distorted depending on $\vec{B}_{\perp}$, the distance traveled by the muons, and their energy distribution. For very large zenith angles the pattern results in two lobes on each side of the shower axis, one for the negatively and one for the positively charged muon components. The magnetic deviation $\delta x$ experienced by muons of different charges is [194]

$$
\delta x=\frac{e B_{\perp} d^{2}}{2 p},
$$

where $e$ is the electron charge. Combining this with Eq. (41) we obtain,

$$
\delta x=0.15\left(\frac{B_{\perp}}{\mathrm{T}}\right)\left(\frac{d}{\mathrm{~m}}\right)\left(\frac{p_{T}}{\mathrm{GeV}}\right)^{-1} \bar{r}=\alpha \bar{r},
$$

where $\bar{r}$ corresponds to the muon deviation in the transverse plane in the absence of a magnetic field, and $\alpha$ measures the ratio of displacement in the transverse plane due to $p_{T}$ as well as the displacement due to the magnetic field. The density of muons in the transverse plane can be obtained by making the transformation

$$
x=\bar{x}+\alpha \sqrt{\bar{x}^{2}+\bar{y}^{2}}, \quad y=\bar{y},
$$


where the barred and unbarred coordinates indicate the position of the muon in the transverse plane in the absence and presence of the geomagnetic field, respectively. The muon number density reads

$$
\rho_{\mu}(x, y)=\bar{\rho}_{\mu}(\bar{x}, \bar{y})\left[\frac{\partial(\overline{x y})}{\partial(x y)}\right]
$$

where $\bar{\rho}_{\mu}(\bar{x}, \bar{y})$ is the density at a distance $\bar{r}=\left(\bar{x}^{2}+\bar{y}^{2}\right)^{1 / 2}$ in the case $\vec{B}=0$ and the last factor is the Jacobian of the transformation.

In a realistic situation the transverse position of the muon $\bar{r}$ is affected by both multiple scattering and the transverse position of the parent pions. Following [194], to account for this effect we use Eq. (41), setting $d$ to a constant and we assume that at a given $\bar{r}$ there is an energy distribution. For convenience one introduces the variable $\epsilon=\log _{10} E_{\mu}$, such that

$$
\langle\epsilon\rangle=A-\gamma \log _{10} \bar{r} \text {. }
$$

The muon density is taken to be

$$
\rho_{\mu}(\bar{r}, \epsilon)=P(\epsilon ;\langle\epsilon\rangle, \sigma) \rho_{\mu}(\bar{r}),
$$

where $P$ is a distribution of mean $\langle\epsilon\rangle$ and standard deviation $\sigma$. Now, one obtains the muon number density in the coordinate system $(x, y)$, by using Eq. (46),

$$
\rho_{\mu}(x, y)=\int \mathrm{d} \epsilon P(\epsilon ;\langle\epsilon\rangle, \sigma) \rho_{\mu}(\bar{r}),
$$

where

$$
\bar{r}=\left[\left(x-\frac{e B_{\perp} d^{2} c}{2 E_{\mu}}\right)^{2}+y^{2}\right]^{1 / 2}
$$

The muon number density given in Eq. (49) depends on three quantities: (i) the distribution of $\epsilon$ that hereafter is taken as a Gaussian with mean given by Eq. (47) and $\sigma \sim 0.4$, (ii) the effective distance to the production point $d$, and (iii) the lateral distribution function of the muons in the transverse plane. Fig. 13 shows fits [194] to the lateral distributions at different zenith angles using the NKG-like LDF,

$$
\rho(\bar{r})=\mathscr{N} \bar{r}^{-\psi}\left[1+\frac{\bar{r}}{\mathscr{R}}\right]^{-\kappa}
$$

with $N, \psi, \kappa$, and $\mathscr{R}$ as given in Table 1 , and $d$ taken as 16,32 , and $88 \mathrm{~km}$, for $\theta=60^{\circ}, 70^{\circ}$, and $80^{\circ}$, respectively. One can see from the figure that the parametrization reproduce the simulation quite well up to a core distance of $1 \mathrm{~km}$.

\subsubsection{Muon content of the shower tail}

As discussed in the previous section, once the shower particle energies fall below $\epsilon_{0}$, ionization losses take over from other electromagnetic processes, and the number of electrons and photons in the shower begins to decrease, while the number of muons remains more-or-less the same. We will refer to this region of the shower as the "tail." Most ground arrays are located below the altitude at which $X_{\max }$ 


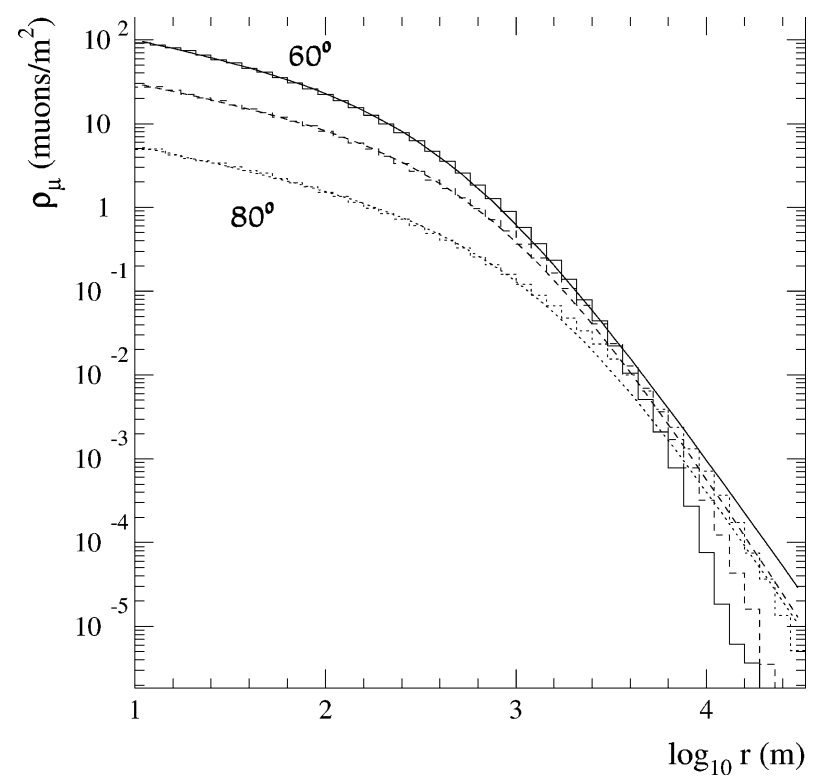

Fig. 13. Lateral distribution of muons from AIRES simulations superimposed over the best fits obtained using Eq. (51). From top to bottom the curves correspond to $60^{\circ}, 70^{\circ}$, and $80^{\circ}$ [194].

Table 1

Best values for the parameters in Eqs. (47) and (51) as obtained from fits to Monte Carlo simulations, using SIBYLL 1.6 to process the hadronic interactions

\begin{tabular}{llllllr}
\hline$\theta\left(^{\circ}\right)$ & $A$ & $\gamma$ & $\mathscr{N}$ & $\psi$ & $\kappa$ & $\mathscr{R}(\mathrm{m})$ \\
\hline 60 & $2.67 \pm 0.23$ & $0.75 \pm 0.1$ & 569.9 & 0.52 & 4.05 & 782.8 \\
70 & $4.04 \pm 0.20$ & $0.73 \pm 0.06$ & 227.1 & 0.49 & 4.35 & 1010.0 \\
80 & $3.63 \pm 0.20$ & $0.81 \pm 0.07$ & 78.4 & 0.52 & 4.49 & 1513.0 \\
\hline
\end{tabular}

occurs, even in the case of vertical showers induced by ultra high energy primaries. This means that ground arrays observe predominantly shower tails. In this section, we describe the variation of the shower tail's muon content with energy, and compare the original calculations of Hillas from the early 1970's with more recent detailed Monte Carlo simulations.

Muons are produced when a shower has cooled sufficiently to allow pions to decay before they interact (recall that the probability for decay of a $1 \mathrm{TeV}$ pion in the atmosphere is somewhat less than 10\%). Hillas' group at the University of Leeds reviewed various models for this cooling process and analyzed their consistency with data from emulsion experiments as well as cosmic air shower observations $[195,196]$. They found the data at ground level to be best reproduced by the model (so-called " $\mathrm{E}$ ") which predicted that for $\theta=14^{\circ}$, the number of muons in proton showers scales as

$$
N_{\mu}^{p} \propto E^{0.94}
$$


Interestingly, this result differs only by an offset in the normalization when compared to the prediction from full-blown modern-day Monte Carlo simulations, as shown in Fig. 14. Of course, the exponent in Eq. (52) varies with zenith angle. It is possible to take into account the zenith angle dependence either through the "constant intensity cut" method [198], or by simply determining the behavior of the exponent as a function of zenith angle [182]. Furthermore, it has been recently noted that this exponent is more accurately taken to have a logarithmic energy dependence [86].

The muon content of EAS at ground level $N_{\mu}$, as well as the ratio $N_{\mu} / N_{\mathrm{e}}$, are sensitive to primary composition (here, $N_{\mathrm{e}}$ is the electron content at ground level). To estimate the ratio of the muon content of nucleus induced to proton induced showers, we can resort again to the principle of superposition. Using Eq. (52) we find that the total number of muons produced by the superposition of $A$ individual proton showers is, $N_{\mu}^{A} \propto A\left(E_{A} / A\right)^{0.94}$. Consequently, in a vertical shower, one expects a cosmic ray nucleus to produce about $A^{0.06}$ more muons than a proton. This implies that a shower initiated by an iron nucleus produces about $27 \%$ more muons than a proton shower. Note, however, that a change in the hadronic interaction model could produce a much larger effect than a change in the primary species. For example, replacing QGSJET 01 with SIBYLL 1.6 as the hadronic interaction model leads to a prediction of $60 \%$ more muons for an iron shower than for a proton shower [182].
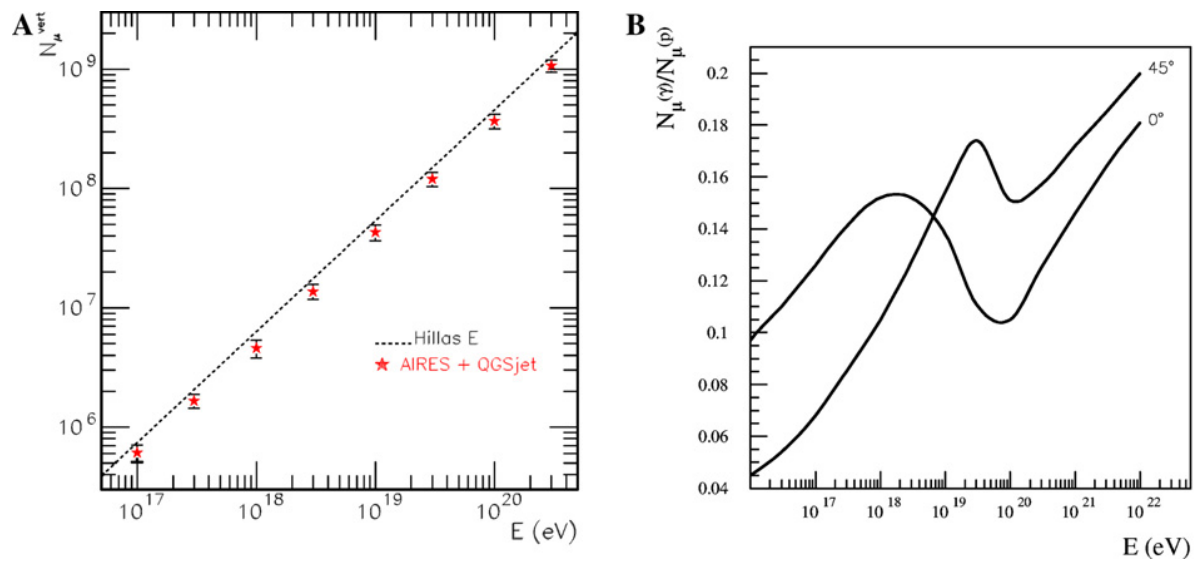

Fig. 14. (A) Total number of muons at ground level as a function of the shower energy. The dashed line indicates the Hillas parametrization for model "E," with a threshold energy set to that of the SUGAR experiment [26]. (For vertical showers considering the SUGAR's energy threshold, one obtains an exponent 0.93 rather than the 0.94 used in the text.) The stars illustrate the results obtained from simulations carried out with AIRES + QGSJET 01, assuming proton primaries. The particles were injected vertically and the observation level was placed at $250 \mathrm{~m}$ above sea level. Muons with energies below the threshold $0.75 \mathrm{GeV}$ are not taken into account in the simulations [183]. If the hadronic interactions are modeled with SIBYLL 1.6 rather than QGSJET 01, an exponent of 0.88 best fits the simulation [197]. (B) Ratio of the muon content for EAS produced by primary gammas and protons. The geomagnetic field is set to the PAO Southern site [150]. 
The situation for $\gamma$-induced showers is a bit different. In this case the muon component of the shower does not simply follow Eq. (52) because of the LPM and geomagnetic field effects [150]. Competition between the two processes leads to a complex behavior in $N_{\mu}^{\gamma} / N_{\mu}^{p}$, as shown in Fig. 14.

In this section, we have described the four main energy loss mechanisms for muons en route through the atmosphere. The rate of energy attenuation for muons is much smaller than it is for electrons, and the energy loss processes are only really of interest in the case of extremely inclined showers for which the original electromagnetic component is mostly absorbed. In such cases, small electromagnetic sub-showers can still arise from bremsstrahlung, pair production, and knock-on electrons. In addition, muon-nucleus interactions induce hadronic sub-showers. We also discussed the effect of different energy loss mechanisms on the electron and muon distributions in time and space. Because they are less subject to multiple scattering, muons tend to arrive at the ground earlier and more compressed in time. The ratio of muons to electrons far from the core is much greater than it is near the core, and this effect is more pronounced at higher zenith angles.

The muon content of the shower tail is quite sensitive to unknown details of hadronic physics. This implies that attempts to extract composition information from measurements of muon content at ground level tend to be systematics dominated. The muon LDF is mostly determined by the distribution in phase space of the parent pions. However, the pionization process together with muon deflection in the geomagnetic field obscures the distribution of the first generation of pions. A combination of detailed simulations, high statistics measurements of the muon LDF, and identification of the primary species using uncorrelated observables could shed light on hadronic interaction models.

\section{Fingerprints of primary species in EAS}

A determination of primary composition is invaluable in revealing the origin of cosmic rays as this information would provide important bounds on sources and on possible production and acceleration mechanisms. In addition, a proper interpretation of anisotropy information requires knowledge of the primary mass due to the influence on propagation of the galactic and intergalactic magnetic fields. Attempting to determine the primary composition of cosmic rays by measuring various shower parameters is fraught with systematic uncertainty. Furthermore, because of the stochastic nature of the extensive air showers, there are inherent shower-toshower fluctuations in measured shower observables that cannot be attributed to experimental systematic error alone. Therefore, the determination of primary composition on an event-by-event basis is an intractable problem. Nevertheless, statistical analyses of shower observables known to correlate with the primary composition are possible. Based on the general signatures of the EAS described in previous sections, we provide a summary of the observables that help to separate primary species. 


\subsection{Photon showers}

In this section, we provide an overview of how the EAS characteristics described in the previous sections allow one to distinguish photon primaries from other species. As discussed in Section 3.1 photon-induced showers are expected to generate fewer muons than baryon-induced showers. This clear signature can be exploited by surface arrays which are equipped with dedicated muon detectors or are capable of distinguishing muons using shower observables sensitive to the muon content. The AGASA Collaboration [199,200] has used the muon content of the detected EAS to set bounds on the percentage of photon primaries present in the observed flux. AGASA comprises 111 stations covering an area of $100 \mathrm{~km}^{2}$. Detectors of 2.8 $10 \mathrm{~m}^{2}$ area, capable of measuring muon densities up to $\approx 10 \mathrm{~m}^{-2}$, were deployed in 27 stations. The analysis of the AGASA Collaboration, which takes into account the LPM effect and conversion in the geomagnetic field, shows that at the $95 \%$ $\mathrm{CL}$, the fraction of $\gamma$-rays above $10^{10}, 10^{10.25}$, and $10^{10.5} \mathrm{GeV}$ is less than 34,59 , and $63 \%$, respectively. Of course, these bounds depend on the hadronic interaction models used to simulate the showers. Several models were used in this analysis, and the reported limits are the least restrictive ones.

Another powerful tool for discriminating between photons and baryons using data collected by surface detectors relies on comparing the flux of vertical showers to that of inclined showers, a technique which exploits the attenuation of the electromagnetic shower component for large slant depths. As an illustration of this technique, we describe below the constraints on the $\gamma$-ray flux obtained from Haverah Park measurements [201]. The first crucial ingredient in the analysis is the vertical flux normalization. This should be determined in a way which is free from systematic uncertainties associated with the primary composition. Fluorescence detectors, which record "calorimetric" measurements, provide the best tool to attain this normalization, and in the analysis described here the data from Fly's Eye [41] were used [202]. From this known vertical spectrum and Monte Carlo simulations of shower propagation and detector response, a prediction can be made for the expected rate of inclined events for each type of primary. For inclined showers in the zenith angle range $60^{\circ}<\theta<80^{\circ}$, the Haverah Park experiment collected 46 events with energy above $10^{10} \mathrm{GeV}$ and 7 events above $10^{10.6} \mathrm{GeV}$. A comparison of these observations to the results extracted from simulations is shown Fig. 15. If one assumes the primary spectrum comprises a mixture of protons and photons, then the Haverah Park data imply that above $10^{10} \mathrm{GeV}$, less than $30 \%$ of this admixture can be photons, and above $10^{10.6} \mathrm{GeV}$ less than $55 \%$ can be photons. Both of these statements are made at the 95\% CL [201]. Even though Fly's Eye provides a flux measurement which is independent of the mass composition, one should be aware of the inherent systematic uncertainties in aperture estimates of fluorescence detectors. A separate normalization technique using both fluorescence and surface array data leads to bounds within $20 \%$ of the previous estimates [197].

The sensitivity of PAO for isolating $\gamma$-ray primaries using this method was estimated in [203]. Given the huge statistics - above $60^{\circ}$ the aperture of the observatory is increased by almost a factor 2-PAO will place severe constraints on the photon 


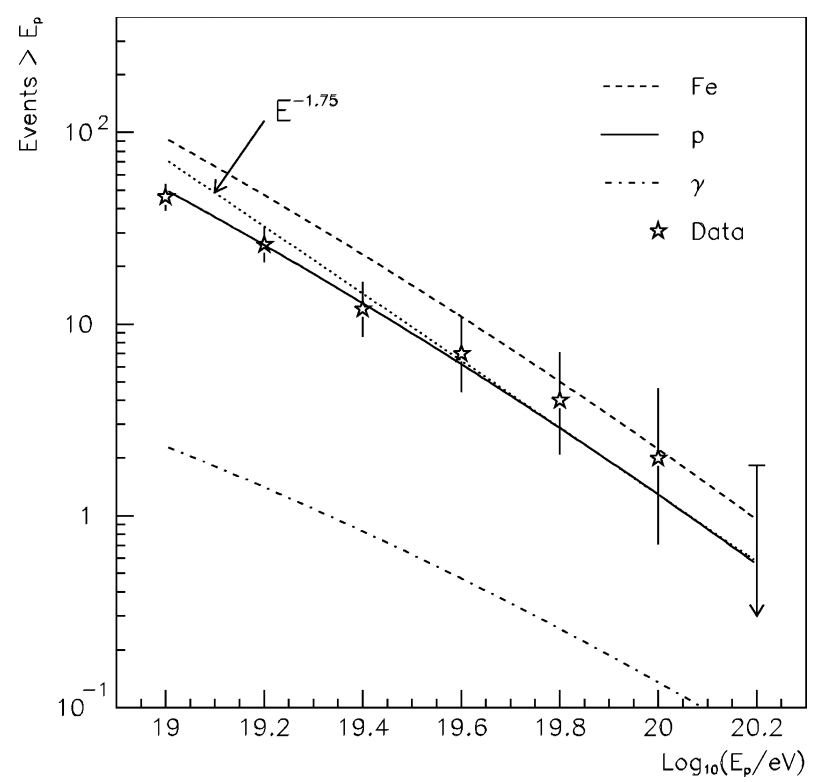

Fig. 15. Integral number of inclined events as a function of energy for the Haverah Park data set compared with the predictions for iron, proton, and photon primaries. Here the energy is calculated assuming a proton primary. The slope of the assumed primary spectrum $\propto E^{-1.75}$ is shown to illustrate the increase of triggering efficiency with energy [201].

content of the observed flux. Additionally, hybrid techniques available to PAO will facilitate independent cross-checks on the systematic uncertainties.

The effect of the geomagnetic field on photons also leads to an energy dependence and characteristic anisotropy in the directional distribution of the fraction of events with abnormally deep profiles which are not compatible with proton or nuclei primaries. This technique has not yet been implemented in the analysis of real data, but the potential for the HiRes and PAO experiments has been evaluated $[155,154]$.

\subsection{Hadronic primaries}

We now discuss how baryonic species may, to some extent, be distinguished by the signatures they produce in the atmosphere. As in the previous section, we consider both surface array and fluorescence detector observables.

As mentioned in Section 3.1, the main purpose of fluorescence detectors is to measure the properties of the longitudinal development. The shower longitudinal profile can be parameterized using the Gaisser-Hillas function [204]

$$
N_{\mathrm{e}}(X)=N_{\mathrm{e}, \max }\left(\frac{X-X_{1}}{X_{\max }-X_{1}}\right)^{\left[\left(X_{\max }-X_{1}\right) / \lambda\right]} \exp \left\{\frac{X_{\max }-X}{\lambda}\right\}, \quad X \geqslant X_{1},
$$


where $N_{\mathrm{e}, \max }$ is the size at the maximum, $X_{1}$ is the depth of the first observed interaction, and $\lambda$ is a floating parameter in the fit, generally fixed to $70 \mathrm{~g} / \mathrm{cm}^{2}$. Using this parametrization, fluorescence detectors can measure $X_{\max }$ with a statistical precision typically around $30 \mathrm{~g} / \mathrm{cm}^{2}$.

The speed of shower development is the clearest indicator of the primary composition. It was shown in Section 3.1 using the superposition model that there is a difference between the depth of maximum in proton and iron induced showers. In fact, nucleus-induced showers develop faster, having $X_{\max }$ higher in the atmosphere. From Monte Carlo simulations, one finds that the difference between the average $X_{\max }$ for protons and iron nuclei is about $90-100 \mathrm{~g} / \mathrm{cm}^{2}$. However, because of shower-to-shower fluctuations, it is not possible to obtain meaningful composition estimates from $X_{\max }$ on a shower-by-shower basis, though one can derive composition information from the magnitude of the fluctuations themselves. For protons, the depth of first interaction fluctuates more than it does for iron, and consequently the fluctuations of $X_{\max }$ are larger for protons as well. Specifically, the standard deviation $\sigma\left(X_{\max }\right)$ is $53 \mathrm{~g} / \mathrm{cm}^{2}$ for protons and $22 \mathrm{~g} / \mathrm{cm}^{2}$ for iron [9]. These fluctuations depend only weakly on the choice of interaction model. The HiRes Collaboration has recently analyzed their stereo data sample in the energy range $E=10^{9.5}-10^{10.4} \mathrm{GeV}$ [205]. The results are shown in Fig. 16, together with the expected distributions using several hadronic interaction models. While agreement between data and Monte
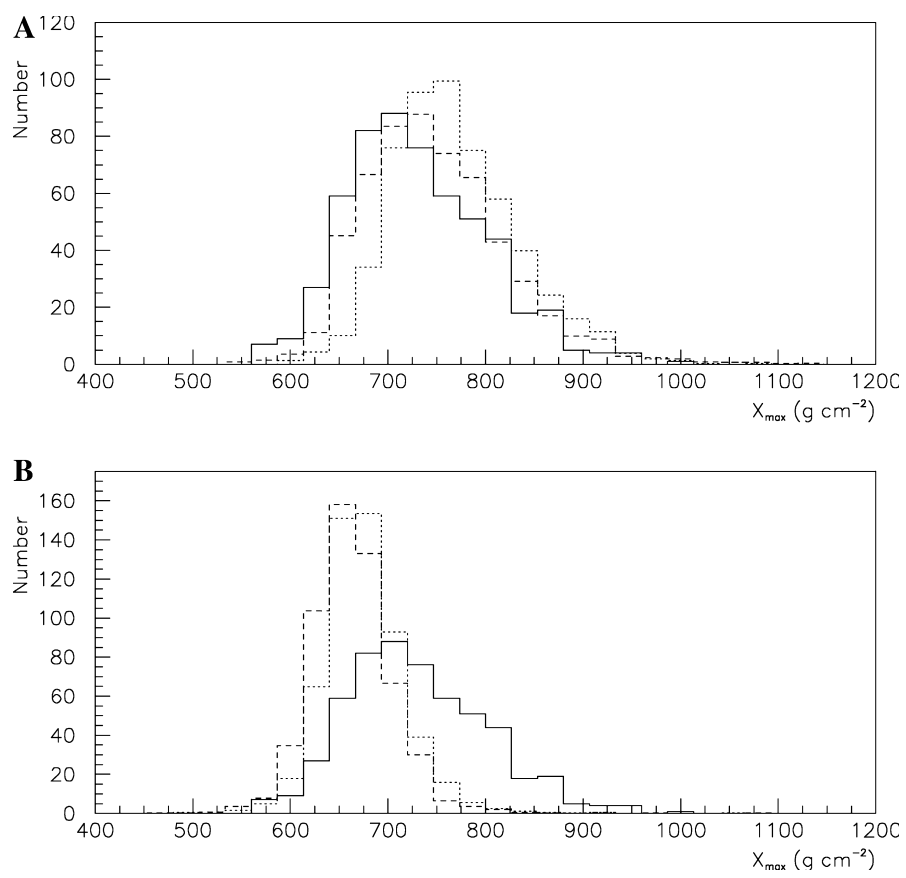

Fig. 16. Distribution of observed $X_{\max }$ from HiRes stereo data for showers in the energy range $10^{9.5}$ $10^{10.4} \mathrm{GeV}$ (solid histogram). The predictions for proton (A) and for iron (B) are given for QGSJET 01 (dashed histogram) and SIBYLL 2.1 (dotted histogram). This figure is courtesy of Greg Archbold. 
Carlo is not perfect for any of the models, the data do qualitatively suggest a proton dominated composition.

Changes in the mean mass composition of the cosmic ray flux as a function of energy will manifest as changes in the mean values of $X_{\max }$. This change of $X_{\max }$ with energy is commonly known as the elongation rate [206]:

$$
D_{\mathrm{e}}=\frac{\delta X_{\max }}{\delta \ln E} .
$$

For purely electromagnetic showers, $X_{\max }(E) \approx X_{0} \ln \left(E / \epsilon_{0}\right)$ and then the elongation rate is $D_{\mathrm{e}} \approx X_{0}$. For proton primaries, the multiplicity rises with energy, and thus the resulting elongation rate becomes smaller. This can be understood by noting that, on average, the first interaction is determined by the proton mean free path in the atmosphere, $\lambda_{N}$. In this first interaction the incoming proton splits into $\langle n(E)$ secondary particles, each carrying an average energy $E /\langle n(E)\rangle$. Assuming that $X_{\max }(E)$ depends logarithmically on energy, as we found with the Heitler model described in Section 3.1, it follows that:

$$
X_{\max }(E)=\lambda_{N}+X_{0} \ln [E /\langle n(E)\rangle] .
$$

If we assume a multiplicity dependence $\langle n(E)\rangle \approx n_{0} E^{4}$, then the elongation rate becomes,

$$
\frac{\delta X_{\max }}{\delta \ln E}=X_{0}\left[1-\frac{\delta \ln \langle n(E)\rangle}{\delta \ln E}\right]+\frac{\delta \lambda_{N}}{\delta \ln E}
$$

which corresponds to the form given by Linsley and Watson [207],

$$
D_{\mathrm{e}}=X_{0}\left[1-\frac{\delta \ln \langle n(E)\rangle}{\delta \ln E}+\frac{\lambda_{N}}{X_{0}} \frac{\delta \ln \left(\lambda_{N}\right)}{\delta \ln E}\right]=X_{0}(1-B) .
$$

Using the superposition model introduced in Section 3.1 and assuming that

$$
B \equiv \Delta-\frac{\lambda_{N}}{X_{0}} \frac{\delta \ln \lambda_{N}}{\delta \ln E}
$$

is not changing with energy, one obtains for mixed primary composition [207]

$$
D_{\mathrm{e}}=X_{0}(1-B)\left[1-\frac{\partial\langle\ln A\rangle}{\partial \ln E}\right] \text {. }
$$

Thus, the elongation rate provides a measurement of the change of the mean logarithmic mass with energy. One caveat of the procedure discussed above is that Eq. (57) accounts for the energy dependence of the cross section and violation of Feynman scaling only for the first interaction. Note that subsequent interactions are assumed to be characterized by Feynman scaling and constant interaction cross sections, see Eq. (58). Above $10^{7} \mathrm{GeV}$, these secondary interactions play a more important role, and thus the predictions of Eq. (59), depending on the hadronic interaction model assumed, may vary by up to $20 \%$ [86].

For convenience, the elongation rate is often written in terms of energy decades,

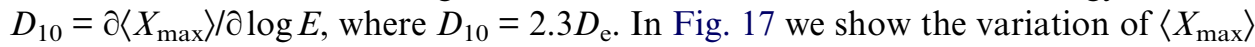
with primary energy as measured by several experiments together with predictions 

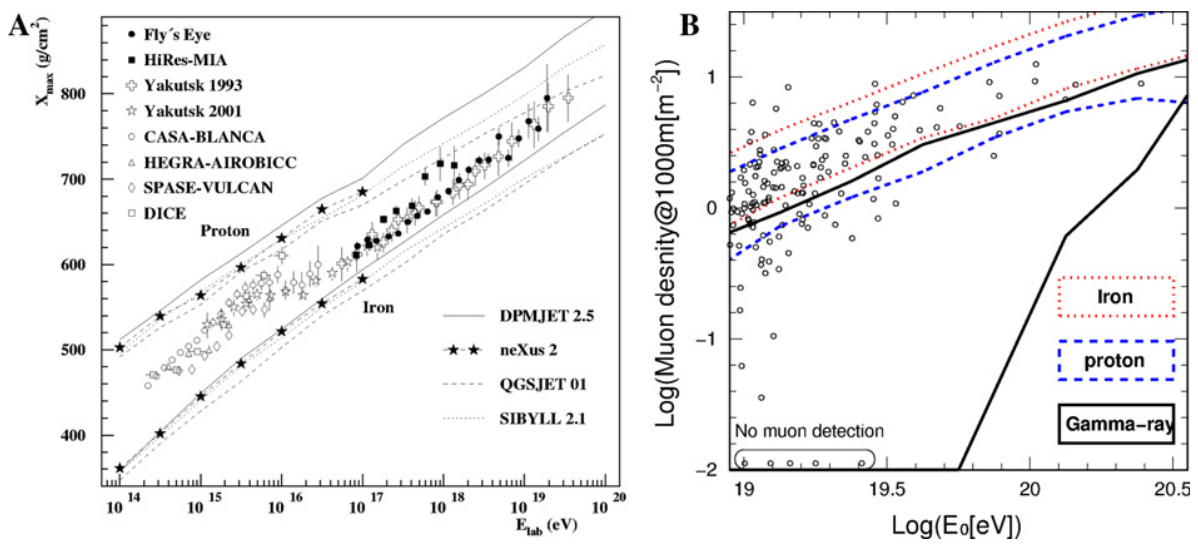

Fig. 17. (A) Variation of $X_{\max }$ with energy as seen by different experiments: Fly's Eye [208], HiRes-MIA [209,210], HEGRA-AIROBICC [211], CASA-BLANCA [212], DICE [213], SPASE-VULCAN [214], and YAKUTSK [215]. The rising curves indicate simulated results for proton and iron primaries using various hadronic interaction models [162]. (B) The circles indicate the experimental measurements of $\log \rho_{\mu}$ at $1000 \mathrm{~m}$ from the core vs. logarithm of the primary energy. The lines indicate the $1 \sigma$ band for iron, proton, and photon predictions [199].

from a variety of hadronic interaction models. For protons and iron, Monte Carlo simulations indicate $D_{10} \approx 55 \mathrm{~g} / \mathrm{cm}^{2}$ and for photons $D_{10} \approx 84 \mathrm{~g} / \mathrm{cm}^{2}$ [9]. Recent results presented by the HiRes Collaboration [205] using stereo data favours a light component above $10^{9} \mathrm{GeV}$, and the reported variation of $\left\langle X_{\max }\right\rangle$ with logarithm of primary energy is $D_{10}=54.5 \pm 6.5$, consistent with a constant or slowly changing composition between $10^{9}$ and $10^{10.4} \mathrm{GeV}$.

As an attentive reader should know by now, the muon content of the shower is strongly sensitive to the nature of the primary. The AGASA Collaboration used measurements of the muon component to discern the primary composition [199]. For events with estimated energy $>10^{10} \mathrm{GeV}$, and zenith $\leqslant 36^{\circ}$, the muon density at $1000 \mathrm{~m}$ from the shower core was used to estimate the primary mass. Expected muon densities for iron and proton primaries were estimated from Monte Carlo simulations, and comparison of the expected to observed densities suggests a dominance of light composition. Specifically, above $10^{10} \mathrm{GeV}$ the average fraction of iron is $14_{-14}^{+16 \%}$, rising to $30_{-6}^{+7} \%$ above $10^{10.25} \mathrm{GeV}$, and above $10^{10.5} \mathrm{GeV}$ the fraction is found to be below $66 \%$ at the $1 \sigma$ level. In Fig. 17 we show the distributions of muon density at $1000 \mathrm{~m}$ from the core as a function of primary energy as reported by the AGASA Collaboration, together with the predictions for $\pm 1 \sigma$ bounds for iron, proton and photon induced showers.

The steepness of the lateral distribution of particles at ground level also correlates with the depth of shower maximum, and thus carries information about the primary species. For instance, a proton-initiated shower would have a steeper average lateral distribution, since the shower develops deeper in the atmosphere than an ironinitiated shower of the same energy. Recently, this approach has been used in 
conjunction with the latest shower and detector simulation codes to re-interpret the lateral distribution measurements from Haverah Park [216] and Volcano Ranch [217-219]. In the case of the Volcano Ranch array, 80 scintillators were laid out in a grid with a separation of $147 \mathrm{~m}$, facilitating a very fine-grained measurement of the lateral distribution. Recall that the signal measured by plastic scintillators is the average energy loss in the scintillator of electrons, muons, and photons in units of the energy loss of vertically penetrating muons. The corresponding lateral distribution was parameterized by an NKG-like formula [168],

$$
S_{\mathrm{VR}}(r)=\frac{N}{r_{\mathrm{M}}^{2}} \frac{\Gamma(\eta-\alpha)}{2 \pi \Gamma(2-\alpha) \Gamma(\eta-2)}\left(\frac{r}{r_{\mathrm{M}}}\right)^{-\alpha}\left(1+\frac{r}{r_{\mathrm{M}}}\right)^{-(\eta-\alpha)},
$$

where $N$ is the shower size, $\eta$ and $\alpha$ describe the logarithmic slope, and $r_{\mathrm{M}} \simeq 100 \mathrm{~m}$ at Volcano Ranch. For events at median energy $10^{9} \mathrm{GeV}$ and shower sizes $N=4 \times 10^{7}$ $6 \times 10^{9}$, measurements of $\eta$ (with $\alpha$ fixed to 1 ) were analyzed in the range of zenith angle $1.0<\sec \theta<1.1$ [220,221]. These measurements are shown in Fig. 18 along with the recently simulated results for purely proton and iron primaries using QGSJET 98 as the hadronic interaction model. One can clearly see the dependence on primary composition reflected in the distribution of $\eta$. To quantify this dependence, a maximum likelihood technique was employed assuming a bimodal composition of proton and iron. The cosmic ray mass composition, deduced from Volcano Ranch data, resulted in a mean fraction $(89 \pm 5$ (stat) \pm 12 (sys) $) \%$ of iron in the whole energy range $10^{8.7}-10^{10} \mathrm{GeV}$; mean energy $10^{9} \mathrm{GeV}$. The resulting admixture is also shown in Fig. 18. Systematic uncertainties associated with the hadronic interaction model are important in this analysis. If QGSJET 98 is replaced by QGSJET 01, then the fraction of iron changes from $(89 \pm 5) \%$ to $(75 \pm 5) \%$.

As described in Section 3.1 the electromagnetic component of an EAS suffers more scattering and energy loss than the muonic component and consequently, muons tend to arrive earlier and over a shorter period of time. This means that parameters characterizing the time structure of the EAS will be correlated with $X_{\max }$ and hence with primary mass. An early study of the shower signal observed in water
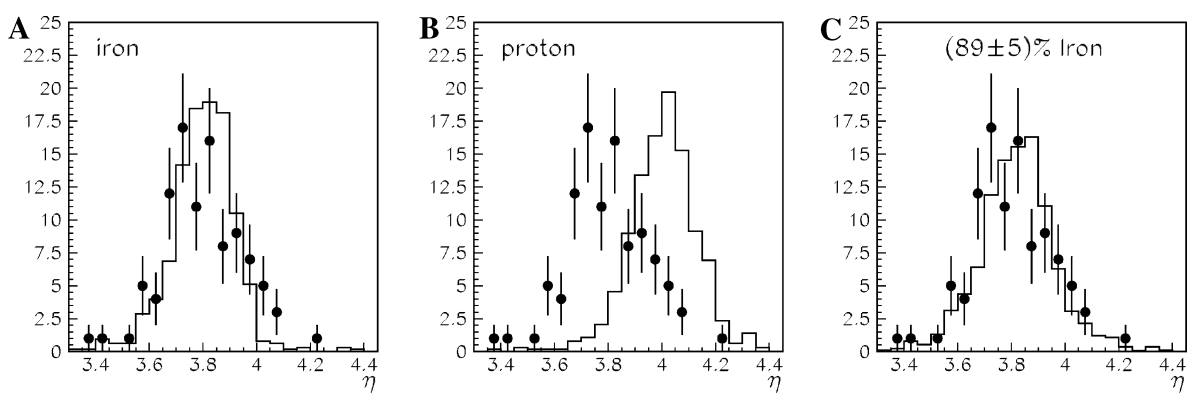

Fig. 18. Comparison of the simulated distribution of $\eta$ (histogram) with measured distributions (points) for iron (A) and proton (B). One can see that an iron-dominated composition best fits the data, but that the addition of a lighter component is needed to fit the distribution at large values of $\eta$. (C) The best composition fit to the measured distribution of $\eta$ (points) from maximum likelihood analysis. 
Čerenkov detectors at the Haverah Park array [222] established the utility of a shower property known as rise time in estimating the primary composition. Specifically, the rise time, $t_{1 / 2}$, is defined as the time for the signal to rise from 10 to $50 \%$ of the full signal. Interestingly, the relation between rise time and depth of maximum also implies a relation between the rise time and the elongation rate. As suggested by Linsley [206] if $P$ represents the average value of some shower parameter, such as the rise time, which does not depend explicitly on primary energy but depends on the depth of observation, $X$, and the depth of shower maximum $X_{\max }$, then the elongation rate can be derived from the following expression:

$$
\left(\frac{\delta P}{\delta \ln E}\right)_{X}=-F D_{\mathrm{e}}\left(\frac{\delta P}{\delta X}\right)_{E},
$$

where $F$ depends on the depth dependence of $P$. For a depth dependence of the form $f\left(X / X_{\max }\right), F=X / X_{\max }$, whereas for $f\left(X-X_{\max }\right), F=1$.

This alternative approach was applied to Haverah Park data using the rise time $t_{1 / 2}$ of the signals [223] to produce a measurement of the elongation rate from $10^{8.3}$ to $10^{11} \mathrm{GeV}$. By means of an experimentally determined value for the dependence of $t_{1 / 2}$ with depth, they obtained an elongation rate of $70 \pm 5 \mathrm{~g} / \mathrm{cm}^{2}$, averaged over the previously mentioned energy range. Their result is suggestive of an evolution to lighter species with rising energy.

Recently, an extension of the work on the shower front thickness using Haverah Park data was performed, focusing on the highest energy events [224]. In this analysis the averaged values of the rise time at a large distance from the core were compared with Monte Carlo (CORSIKA/QGSJET 01) predictions for proton and iron values. The result indicates a large fraction $(\approx 80 \%)$ of iron nuclei at $10^{10} \mathrm{GeV}[225]$.

Azimuthal asymmetries in the size [226] and time structure of signals at the ground [227] have been observed in non-vertical showers. The origin of these asymmetries has been discussed in Section 3.1. The AUGER Collaboration has found that the asymmetry in time distributions offers a new possibility for the determination of mass composition, because its magnitude is strongly dependent on the muon to electromagnetic ratio at the observation level. A preliminary study of the timing information of EAS using simulated proton and iron events was used to estimate the sensitivity of the PAO in discrimination of baryonic primaries [227]. The following observables were analyzed: the rise time (time between 10 and $50 \%$ of the integrated signal), fall time (time between 50 and 90\%), and the time between 10 and $90 \%$ of the signal. The timing variables as a function of the azimuth angle in the shower plane, $\zeta$, at fixed range of core distances for proton and iron showers are shown in Fig. 19. The incoming direction of the shower corresponds to $\zeta=0$. As one can see from the figure, these distributions tend to flatten with increasing primary mass. A first analysis seems to indicate that the fall time would be a better discriminating tool. One expects stronger asymmetries at intermediate core distances, where the electromagnetic component dominates. In Fig. 20, we show the mean rise time and fall time as a function of $\zeta$, for events with energy above $10^{9} \mathrm{GeV}$ collected by the PAO in the radial range $600-1400 \mathrm{~m}$. These results, while preliminary, indicate the promise of this method for composition studies, once large statistics samples become available. 

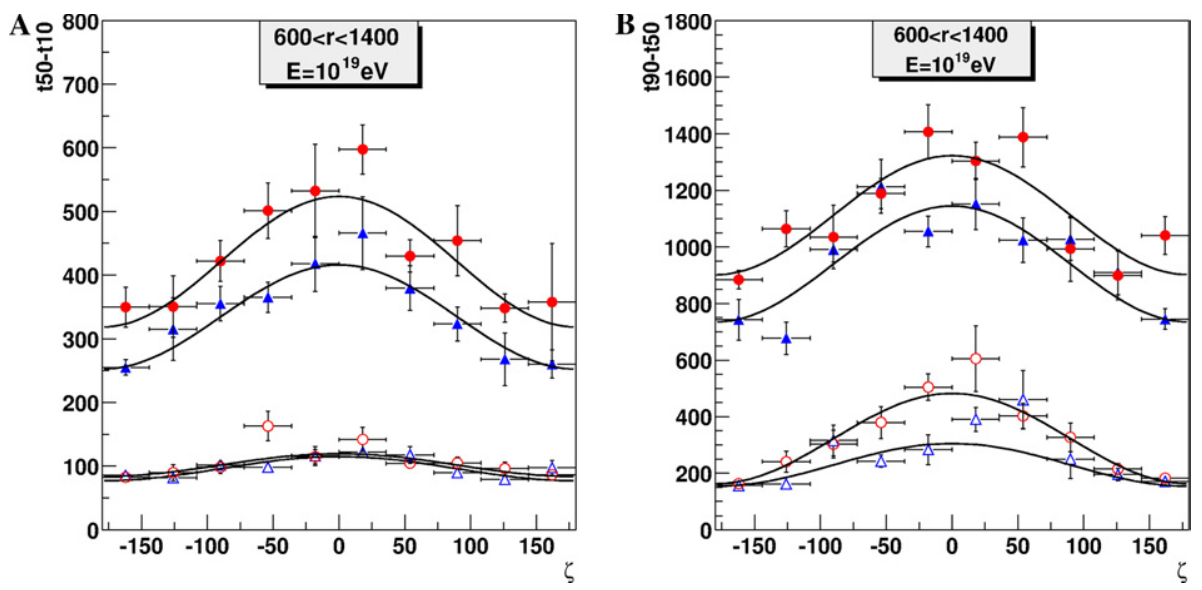

Fig. 19. The triangles (iron) and circles (proton) indicate the rise time (A) and fall time (B) as a function of $\zeta$. Solid symbols correspond to a primary zenith angle of $35^{\circ}$, while the open symbols correspond to $60^{\circ}$ [227].
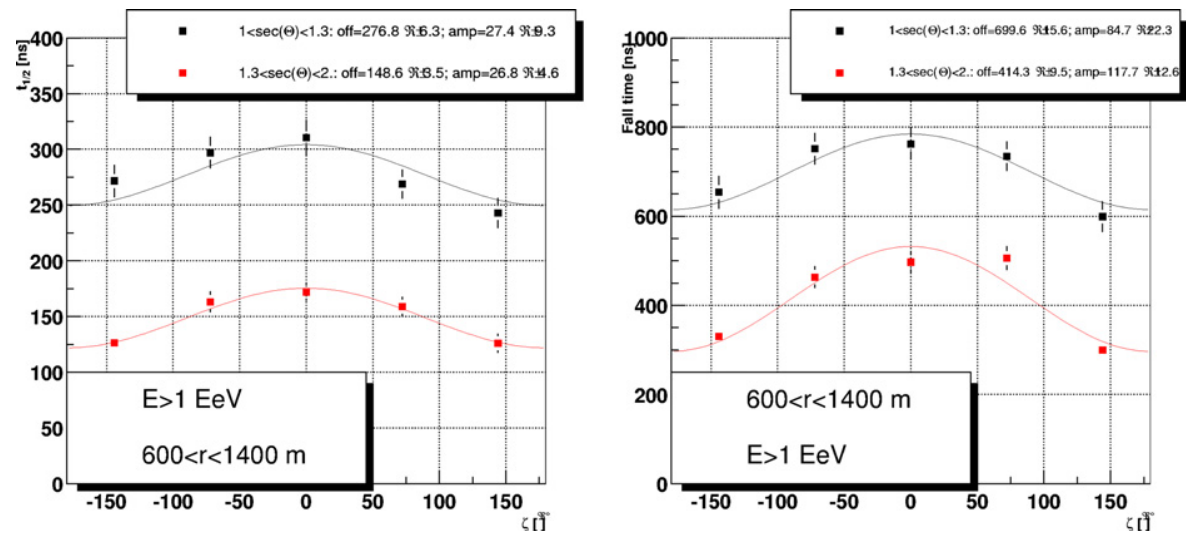

Fig. 20. Time distributions from data collected by the PAO during the period of May to November 2002 in the radial range 600-1400 $\mathrm{m}$ [227]. In each plot the upper points corresponds to $1<\sec \theta<1.3$ while the lower corresponds to $1.3<\sec \theta<2$. A fit to a linear cosine is overlayed on the points, where the fitted parameter "off" is the mean value and "amp" is the amplitude of the asymmetry [227].

In summary, the primary composition has been studied over various energy ranges using several experimental techniques. The variety of results is summarized in Fig. 21, which shows the fraction of iron as a function of energy. Surface arrays such as Haverah Park, Volcano Ranch, and Akeno-AGASA infer $X_{\max }$, and hence the overall composition, from properties of secondary particle distributions at the ground. In this case, the primary source of systematic error arises from uncertainties 


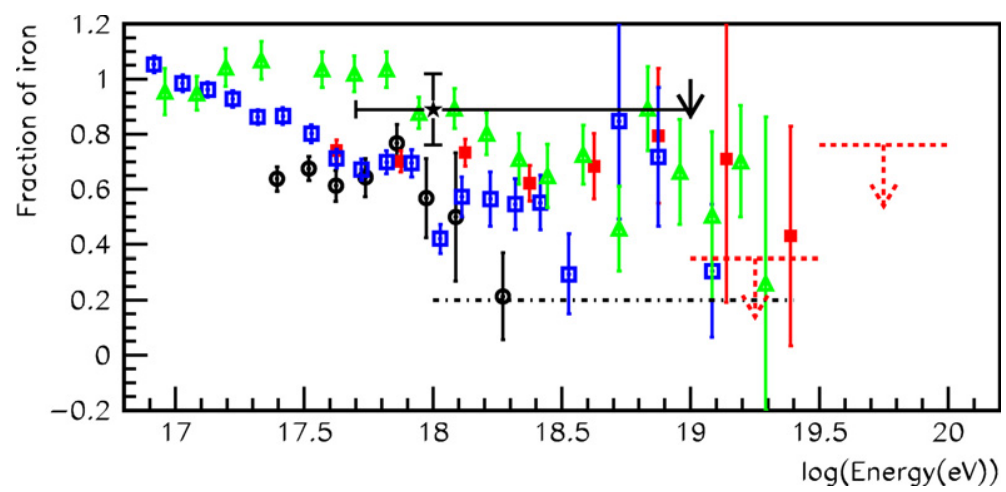

Fig. 21. Iron fraction from various experiments: Fly's Eye $(\triangle)$, AGASA A100 (ם), and AGASA A1 $(\square)$ using SIBYLL 1.6 ([228] and references therein) and Haverah Park [216], using QGSJET98 (O). The mean composition determined in [217] with the corresponding error for the Volcano Ranch energy range using QGSJET98 (认) is shown. The solid line arrow indicates the recent result using rise time measurements from Haverah Park [224]. The dashed arrow lines represents upper limits obtained by the AGASA Collaboration with QGSJET98 [199]. The dot dashed horizontal line corresponds to results reported by the HiRes Collaboration [205].

in the hadronic interaction models. Fluorescence detectors such as Fly's Eye and HiRes observe an image of the longitudinal shower profile and extract $X_{\max }$ directly. Consequently such measurements do not suffer from uncertainties in hadronic event generators, though the data analysis still faces the challenge of assessing atmospheric properties as a function of time. Future stereo data from HiRes and hybrid PAO observations will provide a higher statistics sample with a better control of the systematic uncertainties and will certainly provide clues to the nature of ultra high energy cosmic rays.

\section{Deeply penetrating showers}

Even at large zenith angles, the mean free path of a neutrino in the atmosphere is much larger than the atmosphere's slant depth. However, nearly horizontal showers are especially interesting since in this case the likelihood of interaction is maximized, and furthermore, neutrino induced showers can be easily distinguished from those induced by hadrons high in the atmosphere. In this section, we first consider strategies for detecting these kinds of signatures. After that, we focus attention on recent novel scenarios with $\mathrm{TeV}$-scale quantum gravity and discuss the observables of neutrino showers associated with both sub-planckian and trans-planckian physics.

\subsection{Everyday weakly interacting neutrinos}

Neutrinos are unique and thus far relatively untapped astronomical messengers [229]. Up to now, the only directly observed extraterrestrial neutrinos are those from 
the Sun [230] and from supernova SN1987A [231,232]; these are low energy (MeVrange) neutrinos. Higher energy neutrinos should be generated by cosmic "beam dumps" in which baryonic particles collide with interstellar media. These neutrinos are particularly appealing for astronomy since they are undeflected by magnetic fields and they can travel cosmological distances without interacting [233,234]. In addition to providing a new window for astronomy, cosmic neutrino observations may also enlighten our understanding of fundamental physics. For instance, it will be possible to test Lorentz invariance at extremely high energies and to hunt for exotic processes such as neutrino decay, CPT violation, and small $\delta m^{2}$ oscillations into sterile neutrinos (see e.g. [235-241]). Since neutrinos interact only weakly, very large detector volumes are required, ideally on the order of $1 \mathrm{~km}^{3}$ we [242]. As discussed in the introduction the PAO overlooks $15 \mathrm{~km}^{3}$ we [33], which is sufficient to collect a statistically significant sample of neutrino showers, provided they can be separated from the hadron and photon-induced showers. In what follows we discuss the characteristics of neutrino-induced EAS, and comment on the qualities which may provide a handle to separate these showers from background.

In a typical collision in the Earth's atmosphere the neutrino (with energy $E_{v}$ ) scatters off a proton either via the charged current, $\left(v_{l}, \bar{v}_{l}\right) N \rightarrow\left(l^{-}, l^{+}\right)+$anything, or the neutral current, $\left(v_{l}, \bar{v}_{l}\right) N \rightarrow\left(v_{l}, \bar{v}_{l}\right)+$ anything. ${ }^{10}$ The kinematics of these reactions can be characterized by the inelasticity parameter $y=\left(1-\cos \vartheta^{*}\right) / 2$ and the 4 momentum fraction of the proton carried by the struck quark $x=Q^{2} /(y s)$, where $-Q^{2}$ is the invariant momentum transfer between the incident neutrino and the outgoing lepton. For a given $Q^{2}$ the lowest $x$ is achieved when $y=1$ and the lowest $y$ when $x=1$. Thus, kinematically the small values of $x$ are associated with large values of $y$ and vice versa.

The charged current differential cross section of a neutrino scattering on an isoscalar nucleon $N \equiv(n+p) / 2$ of mass $M$ is given by

$$
\begin{aligned}
\frac{\mathrm{d}^{2} \sigma_{v N}^{\mathrm{CC}}}{\mathrm{d} x \mathrm{~d} y}= & \frac{G_{F}^{2} M E_{v}}{\pi}\left(\frac{m_{W}^{2}}{Q^{2}+m_{W}^{2}}\right)^{2}\left[\frac{1+(1-y)^{2}}{2} F_{2}^{\mathrm{CC}}\left(x, Q^{2}\right)\right. \\
& \left.-\frac{y^{2}}{2} F_{L}^{\mathrm{CC}}\left(x, Q^{2}\right)+y\left(1-\frac{y}{2}\right) x F_{3}^{\mathrm{CC}}\left(x, Q^{2}\right)\right],
\end{aligned}
$$

where $m_{W} \simeq 80.423 \mathrm{GeV}$ denotes the $W$ boson mass, $G_{F}=1.16639 \times 10^{-5} \mathrm{GeV}^{2}$ is the Fermi coupling constant and the structure functions $F_{i}$ in terms of the quark distribution functions of the nucleon $q_{i}\left(x, Q^{2}\right)$ read:

$$
\begin{aligned}
& F_{2}^{\mathrm{CC}}=2 x\left(\frac{d_{v}+u_{v}}{2}+d_{s}+\bar{u}_{s}+s_{s}+\bar{c}_{s}+b_{s}+\bar{t}_{s}\right), \\
& x F_{3}^{\mathrm{CC}}=2 x\left(d_{s}+s_{s}-\bar{u}_{s}-\bar{c}_{s}\right),
\end{aligned}
$$

\footnotetext{
${ }^{10}$ The rate of interaction of $v_{\mathrm{e}}, v_{\mu}, v_{\tau}, \bar{v}_{\mu}, \bar{v}_{\tau}$, with atmospheric electrons is negligible compared to interactions with nucleons. The case of $\bar{v}_{\mathrm{e}} e$ interactions is exceptional because of the intermediate Glashow resonance formed via $W$ boson production at $E_{\bar{v}_{\mathrm{e}}} \approx 10^{6.8} \mathrm{GeV}[243]$.
} 
and

$$
F_{L}^{\mathrm{CC}}=F_{2}^{\mathrm{CC}}-2 x F_{1}^{\mathrm{CC}} \text {. }
$$

Here the subscripts $v$ and $s$ label valence and sea contributions, and $u, d, c, s, t, b$ denote the distributions for various quark flavors in a proton. In the deep inelastic factorization scheme, Eq. (62) can be re-written in terms of quark distributions as $[244,245]$

$$
\frac{\mathrm{d}^{2} \sigma_{v N}^{\mathrm{CC}}}{\mathrm{d} x \mathrm{~d} y}=\frac{2 G_{F}^{2} M E_{v}}{\pi}\left(\frac{m_{W}^{2}}{Q^{2}+m_{W}^{2}}\right)^{2}\left[x q^{\mathrm{CC}}\left(x, Q^{2}\right)+(1-y)^{2} x \bar{q}^{\mathrm{CC}}\left(x, Q^{2}\right)\right],
$$

where

$$
q^{\mathrm{CC}}\left(x, Q^{2}\right)=\frac{u_{v}\left(x, Q^{2}\right)+d_{v}\left(x, Q^{2}\right)}{2}+\frac{u_{s}\left(x, Q^{2}\right)+d_{s}\left(x, Q^{2}\right)}{2}+s_{s}\left(x, Q^{2}\right)+b_{s}\left(x, Q^{2}\right)
$$

and

$$
\bar{q}^{\mathrm{CC}}\left(x, Q^{2}\right)=\frac{u_{s}\left(x, Q^{2}\right)+d_{s}\left(x, Q^{2}\right)}{2}+c_{s}\left(x, Q^{2}\right)+t_{s}\left(x, Q^{2}\right) .
$$

In Eq. (66), we have omitted perturbative QCD corrections, which for $\sqrt{s}>10^{3.4} \mathrm{GeV}$ are insignificant. The average energy loss of this process is $\langle y\rangle \approx 0.2$. Duplicating this procedure, one can straightforwardly obtain the cross section for the neutral current neutrino-nucleon interaction

$$
\frac{\mathrm{d}^{2} \sigma_{v N}^{\mathrm{NC}}}{\mathrm{d} x \mathrm{~d} y}=\frac{G_{F}^{2} M E_{v}}{2 \pi}\left(\frac{m_{Z}^{2}}{Q^{2}+m_{Z}^{2}}\right)^{2}\left[x q^{\mathrm{NC}}\left(x, Q^{2}\right)+(1-y)^{2} x \bar{q}^{\mathrm{NC}}\left(x, Q^{2}\right)\right],
$$

where the quark densities are given by

$$
\begin{aligned}
q^{\mathrm{NC}}\left(x, Q^{2}\right)= & {\left[\frac{u_{v}\left(x, Q^{2}\right)+d_{v}\left(x, Q^{2}\right)}{2}\right]\left[\left(g_{V}^{d}+g_{A}^{d}\right)^{2}+\left(g_{V}^{u}+g_{A}^{u}\right)^{2}\right] } \\
& +2\left[\frac{u_{s}\left(x, Q^{2}\right)+d_{s}\left(x, Q^{2}\right)}{2}\right]\left[\left(g_{V}^{d}\right)^{2}+\left(g_{A}^{d}\right)^{2}+\left(g_{V}^{u}\right)^{2}+\left(g_{A}^{u}\right)^{2}\right] \\
& +2\left[s_{s}\left(x, Q^{2}\right)+b_{s}\left(x, Q^{2}\right)\right]\left[\left(g_{V}^{d}\right)^{2}+\left(g_{A}^{d}\right)\right] \\
& +2\left[c_{s}\left(x, Q^{2}\right)+t_{s}\left(x, Q^{2}\right)\right]\left[\left(g_{V}^{u}\right)^{2}+\left(g_{A}^{u}\right)^{2}\right]
\end{aligned}
$$

and

$$
\begin{aligned}
\bar{q}^{N C}\left(x, Q^{2}\right)= & {\left[\frac{u_{v}\left(x, Q^{2}\right)+d_{v}\left(x, Q^{2}\right)}{2}\right]\left[\left(g_{V}^{d}-g_{A}^{d}\right)^{2}+\left(g_{V}^{u}-g_{A}^{u}\right)^{2}\right] } \\
& +2\left[\frac{u_{s}\left(x, Q^{2}\right)+d_{s}\left(x, Q^{2}\right)}{2}\right]\left[\left(g_{V}^{d}\right)^{2}+\left(g_{A}^{d}\right)^{2}+\left(g_{V}^{u}\right)^{2}+\left(g_{A}^{u}\right)^{2}\right] \\
& +2\left[s_{s}\left(x, Q^{2}\right)+b_{s}\left(x, Q^{2}\right)\right]\left[\left(g_{V}^{d}\right)^{2}+\left(g_{A}^{d}\right)\right] \\
& +2\left[c_{s}\left(x, Q^{2}\right)+t_{s}\left(x, Q^{2}\right)\right]\left[\left(g_{V}^{u}\right)^{2}+\left(g_{A}^{u}\right)^{2}\right] .
\end{aligned}
$$


Here, $m_{Z} \simeq 91.187 \mathrm{GeV}$ is the mass of the neutral intermediate boson and

$$
\begin{aligned}
& g_{V}^{d}=-\frac{1}{2}+\frac{2}{3} \sin ^{2} \theta_{\mathrm{W}}, \quad g_{A}^{d}=-\frac{1}{2}, \\
& g_{V}^{u}=\frac{1}{2}-\frac{4}{3} \sin ^{2} \theta_{\mathrm{W}}, \quad g_{A}^{u}=\frac{1}{2},
\end{aligned}
$$

are the vector and axial-vector couplings for down- and up-type quarks, respectively; with $\sin ^{2} \theta_{\mathrm{W}} \simeq 0.226$ the weak mixing parameter [137]. Similar calculations lead to the cross sections for $\bar{v} N$ scattering. For further details see e.g. [246].

The $x-Q^{2}$ region probed by ultra high energy neutrinos

$$
x \sim \frac{m_{\mathrm{W}}^{2}}{s\langle y\rangle} \sim 3.2 \times 10^{4}\left(\frac{s}{\mathrm{GeV}^{2}}\right)^{-1} \sim 10^{-7},
$$

is well beyond the region accessible by the HERA experiments (see Fig. 3). As we discussed in Section 2, in the renormalization group-improved parton model, the structure functions are extrapolated to very low $x$ considering leading order (LO), next to leading order (NLO), and/or double-leading-logarithmic evolution of DGLAP equations. Using the CTEQ4 pdf's [247] one finds [248]:

$$
\begin{aligned}
& \sigma_{v N}^{\mathrm{CC}}=5.53\left(\frac{E_{v}}{\mathrm{GeV}}\right)^{0.363} \mathrm{pb}, \\
& \sigma_{v N}^{\mathrm{NC}}=2.31\left(\frac{E_{v}}{\mathrm{GeV}}\right)^{0.363} \mathrm{pb}, \\
& \sigma_{\bar{v} N}^{\mathrm{CC}}=5.52\left(\frac{E_{v}}{\mathrm{GeV}}\right)^{0.363} \mathrm{pb},
\end{aligned}
$$

and

$$
\sigma_{\bar{v} N}^{\mathrm{CC}}=2.29\left(\frac{E_{v}}{\mathrm{GeV}}\right)^{0.363} \mathrm{pb} .
$$

For $10^{7} \mathrm{GeV} \lesssim E_{v} \lesssim 10^{12} \mathrm{GeV}$, these cross sections are correct to about $10 \%$, which is smaller than the systematic uncertainties that cosmic ray experiments typically contend with. Note that the reason this uncertainty is small compared to the uncertainty in the cross section shown in Fig. 5 is a consequence of the existence of two viable models for cross section extrapolation in the case of $p p$ interactions. Neutrino interaction lengths

$$
L=1.7 \times 10^{7} \mathrm{~km} \mathrm{we}\left(\frac{\mathrm{pb}}{\sigma_{(\bar{v} v) N}^{(\mathrm{CN}) \mathrm{C}}}\right)
$$

are therefore far larger than the Earth's atmospheric depth, which is only $0.36 \mathrm{~km}$ we even when traversed horizontally. As a consequence, neutrino showers, unlike baryon or photon induced showers, can begin deep in the atmosphere. So, to obtain 
a clean signal of neutrino-induced showers one should be able to identify deeply developing cascades in the whole sample of EAS.

For large zenith angles $\left(\theta>70^{\circ}\right)$, an air shower initiated by a neutrino can be distinguished from that of an ordinary hadron by its shape. As discussed in Section 3, baryons interact high in the atmosphere. Consequently, at ground level the electromagnetic part of these inclined showers is totally extinguished (more than six equivalent vertical atmospheres were gone through) and only the muon channel survives. Besides, the shower front is extremely flat (radius of curvature $>100 \mathrm{~km}$ ) and the particle time spread is very narrow $(\Delta t<50 \mathrm{~ns})$. Since neutrinos can interact deeply in the atmosphere, they can initiate showers in the volume of air immediately above the detector. Therefore, quasi-horizontal showers initiated by neutrinos would still present a curved front (radius of curvature of a few $\mathrm{km}$ ), with particles well spread over time, $\mathcal{O}(\mu \mathrm{s})$, allowing a good characterization of the cascade against background.

If the primaries are mainly electronic and muonic neutrinos, as expected from pion decays, two types of neutrino showers can be distinguished: "mixed" (with full energy) or "pure hadronic" (with reduced energy), respectively [249]. In the charged current interaction of a $v_{\mathrm{e}}$, an ultra high energy electron having about $80 \%$ of the $v_{\mathrm{e}}$ energy is produced and initiates a large electromagnetic cascade parallel to the hadronic cascade. In contrast, the charged current interaction of a $v_{\mu}$ produces a muon which is not easily detectable by existing experiments. In the presence of maximal $v_{\mu} / v_{\tau}$-mixing, $v_{\tau}$-showers must also be considered. The $\tau$ mean flight distance is $\sim 50 E_{\tau} /\left(10^{9} \mathrm{GeV}\right) \mathrm{km}$, whereas the distance between the position of first impact and ground is $\sim 30 \mathrm{~km}$, hence only $\tau$ 's with energy $\lesssim 10^{8.9} \mathrm{GeV}$ will decay before reaching the ground. Since the $\tau$ is produced with about $80 \%$ of the $v_{\tau}$ energy, showers initiated by ultra high energy $v_{\tau}$ 's will be indistinguishable from $v_{\mu}$ showers.

Another interesting category of neutrino-related showers comprises events in which a neutrino skims the Earth, traveling at a low angle along a chord with length of order its interaction length [250,251]. Some of these Earth-skimmers may be converted into charged leptons in the Earth's crust. Unlike electrons that do not escape from rocks, at the energies of interest, muons and $\tau$ leptons travel up to $\mathcal{O}(10 \mathrm{~km})$ inside the Earth. Although muons do not produce any visible signal in the atmosphere, $\tau$ s can produce clear signals for both fluorescence eyes [252] and surface arrays [253] if they decay above the detector. The phenomenon would thus increase the $v$-event rate and enhance the sensitivity of the PAO to neutrino fluxes.

Up to now we have only considered signals one might expect from Standard Model (SM) processes. Many scenarios with new physics beyond the electroweak scale, $M_{\mathrm{EW}}$, have been proposed, some of which increase the weak interaction cross section (see e.g. [254-257]), and hence would have observable implications. As an example of such SM extensions, we consider in the last part of this review a scenario which has generated a great deal of recent interest.

\subsection{Neutrino interactions mediated by gravity}

A promising route towards reconciling the apparent mismatch of the fundamental scales of particle physics and gravity is to modify the short distance behavior of 
gravity at scales much larger than the Planck length. This can be accomplished in a straightforward manner [258-260] if one assumes that the SM fields are confined to a four-dimensional world (corresponding to our apparent universe), while gravity lives in a higher dimensional space. One virtue of this assumption is that very large extra dimensions are allowed without conflicting with current experimental bounds, leading to a fundamental Planck mass much lower than its effective four-dimensional value. In particular, if spacetime is taken as a direct product of a four-dimensional spacetime and a flat spatial $n$-dimensional torus $T^{n}$ (of common linear size $2 \pi r_{c}$ ), one obtains a definite representation of this picture in which the effective four-dimensional Planck scale, $M_{\mathrm{Pl}} \sim 10^{19} \mathrm{GeV}$, is related to the fundamental scale of gravity, $M_{D}$, according to $M_{\mathrm{Pl}}^{2}=8 \pi M_{D}^{n+2} r_{c}^{n}$ [258]. Now, a straightforward calculation shows that, for $n=1$, low-scale gravity within toroidal compactifications is excluded, as gravity would be modified at the scale of our solar system. Astrophysical constraints require $M_{D} \gg 10 \mathrm{TeV}$ for $n=2,3$ and $M_{D} \gtrsim 4 \mathrm{TeV}$ for $n=4$ [261]. For $n \geqslant 5$, however, $M_{D}$ may be as low as a TeV [262-266].

From our four-dimensional point of view the massless graviton appears as an infinite tower of Kaluza-Klein (KK) modes, of which the lowest is the massless graviton itself, but the others are massive. The mass squared of each KK graviton mode reads, $m^{2}=\sum_{i=1}^{n} \ell_{i}^{2} / r_{c}^{2}$, where the mode numbers are $\ell_{i} \in \mathbb{Z}$. Note that the weakness of the gravitational interaction is partially compensated by the tower of $\mathrm{KK}$ modes that are exchanged: the square coupling $M_{\mathrm{Pl}}^{-2}$ of the graviton vertex is exactly cancelled by the large multiplicity of KK excitations $\sim s^{n / 2} r_{c}^{n}$, so that the final product is $\sim s^{n / 2} / M_{D}^{2+n}$. Indeed, if one includes in the interaction the brane Goldstone modes, a form factor $\sim \mathrm{e}^{-m^{2}} / M_{D}^{2}$ is introduced at each graviton vertex [267]. This exponential suppression, which parametrizes the effects of a finite brane tension, provides a dynamical cutoff in the (otherwise divergent) sum over all KK contributions to a given scattering amplitude. Altogether, one may wonder whether the rapid growth of the cross section with energy in neutrino-nucleon reactions mediated by spin 2 particles carries with it observable deviations from SM predictions. ${ }^{11}$

A simple Born approximation to the elastic neutrino-parton cross section (which underlies the total neutrino-proton cross section) leads, without modification, to $\hat{\sigma}_{\mathrm{el}} \sim \hat{s}^{2}[268,269]$. Unmodified, this behavior by itself eventually violates unitarity. This may be seen either by examining the partial waves of this amplitude, or by studying the high energy Regge behavior of an amplitude $A_{R}(\hat{s}, \hat{t}) \propto \hat{s}^{\alpha(\hat{t})}$ with spin-2 Regge pole, viz., intercept $\alpha(0)=2$. For the latter, the elastic cross section is given by

$$
\frac{\mathrm{d} \hat{\sigma}_{\mathrm{el}}}{\mathrm{d} \hat{t}} \sim \frac{\left|A_{R}(\hat{s}, \hat{t})\right|^{2}}{\hat{s}^{2}} \sim \hat{s}^{2 \alpha(0)-2} \sim \hat{s}^{2},
$$

whereas the total cross section reads as

$$
\hat{\sigma}_{\text {tot }} \sim \frac{\mathfrak{I m}\left[A_{R}(\hat{s}, 0)\right]}{\hat{s}} \sim \hat{s}^{\alpha(0)-1} \sim \hat{s}
$$

\footnotetext{
${ }^{11}$ In what follows we only take into account KK excitations on the gravity sector without considering string recurrences of any other field. For a treatment of the latter see e.g. [256].
} 
so that eventually, $\hat{\sigma}_{\mathrm{el}}>\hat{\sigma}_{\text {tot }}$ [270]. Eikonal unitarization schemes modify these behaviors. Specifically, for large impact parameter, a single Regge pole exchange amplitude yields $\hat{\sigma}_{\text {tot }} \sim \ln ^{2}\left(\hat{s} / s_{0}\right)$ [271,272]. For short impact parameters, partial wave unitarity is a tall order as corrections to the eikonal amplitude are expected to become important. Note that graviton self interactions carry factors of $\hat{t}$ associated to the vertices, and thus as $\hat{t}$ increases, so too does the attraction among the scattered particles. Eventually it is expected that gravitational collapse to a black hole $(\mathrm{BH})$ will take place, absorbing the initial state in such a way that short distance effects will be screened by the appearance of a horizon [273-276]. ${ }^{12}$

According to the Thorne's hoop conjecture [278], a BH forms in a two-particle collision when and only when the impact parameter is smaller than the radius of a Schwarzschild $\mathrm{BH}$ of mass equal to $\sqrt{\hat{s}} \equiv \sqrt{x s}$. The conjecture thus predicts a total cross section for BH production proportional to the area subtended by a "hoop"

$$
\hat{\sigma}_{B H}=F(n) \pi r_{s}^{2}(\sqrt{\hat{s}})
$$

of radius $[279,280]$

$$
r_{s}(\sqrt{\hat{s}})=\frac{1}{M_{D}}\left[\frac{\sqrt{\hat{s}}}{M_{D}}\right]^{\frac{1}{1+n}}\left[\frac{2^{n} \pi^{(n-3) / 2} \Gamma\left(\frac{n+3}{2}\right)}{n+2}\right]^{\frac{1}{1+n}},
$$

where $F(n)$ is a constant of order unity. Recent progress has confirmed the validity of Eq. (82) and evaluated the dimension-dependent constant $F(n)$, analytically in four dimensions [281] and numerically in higher dimensions [282]. Of course, this work is purely in the framework of classical general relativity, and is expected to be valid for energies far above the Planck scale, for which curvature is small outside the horizon and strong quantum effects are hidden behind the horizon. Extending it to the planckian regime of center-of-mass energies close to $M_{\mathrm{D}}$ requires a better understanding of quantum gravity than we now possess. Thus it is important to impose a cutoff on the mass of microscopic BHs for which Eq. (82) can reasonably be expected to hold.

In the course of collapse a certain amount of energy is radiated in gravitational waves by the multipole moments of the incoming shock waves, leaving only a fraction $y \equiv M_{\mathrm{BH}} / \sqrt{\hat{s}}$ available to Hawking evaporation, where $M_{\mathrm{BH}}$ is a lower bound on the final mass of the $\mathrm{BH}$. This ratio depends on the classical impact parameter $b$, and so the inclusive production of BHs proceeds through different final states for different values of $b$. These final states are characterized by the fraction $y(z)$ of the initial parton center-of-mass energy which is trapped within the horizon. Here, $z=b / b_{\max }$, and $b_{\max } / r_{s}(\sqrt{\hat{s}})$ is given in Table 2 [282]. With a lower cutoff $M_{\mathrm{BH} \text {,min }}$ on the $\mathrm{BH}$ mass required for the validity of the semi-classical description, this implies a joint constraint

$$
y(z) \sqrt{\hat{s}} \geqslant M_{\mathrm{BH}, \min }
$$

\footnotetext{
12 This paper does not purport to be a comprehensive review of TeV-scale gravity BHs; for an up-to-date and detailed discussion of the topic, the reader is referred to [277].
} 
Table 2

Gravitational collapse parameters

\begin{tabular}{lllllll}
\hline$n$ & 2 & 3 & 4 & 5 & 6 & 7 \\
\hline$b_{\max } / r_{s}(\sqrt{\hat{s}})$ & 1.052 & 1.118 & 1.166 & 1.206 & 1.238 & 1.264 \\
$F(n)$ & 1.341 & 1.515 & 1.642 & 1.741 & 1.819 & 1.883 \\
\hline
\end{tabular}

on the parameters $x$ and $z$. Because of the monotonically decreasing nature of $y(z)$, Eq. (84) sets an upper bound $\bar{z}(x)$ on the impact parameter for fixed $x$. The corresponding parton-parton $\mathrm{BH}$ cross section is $\hat{\sigma}_{\mathrm{BH}}(x)=\pi \bar{b}^{2}(x)$, where $\bar{b}=\bar{z} b_{\max }$. The total $\mathrm{BH}$ production cross section is then [266]

$$
\sigma_{v N \rightarrow \mathrm{BH}}\left(E_{v}, M_{\mathrm{BH}, \min }, M_{D}\right) \equiv \int_{\frac{M_{\mathrm{BH}, \min }^{2}}{y^{2}(0) s}}^{1} \mathrm{~d} x \sum_{i} f_{i}(x, Q) \hat{\sigma}_{\mathrm{BH}}(x),
$$

where $i$ labels parton species and the $f_{i}(x, Q)$ are pdf's (to derived the $\mathrm{BH}$ production cross sections shown in Fig. 22 we used the CTEQ6M pdf's [283,284]). The momentum scale $Q$ is taken as $r_{s}^{-1}$, which is a typical momentum transfer during the gravitational collapse [285]. In contrast to $\mathrm{SM}$ processes, $\mathrm{BH}$ production is not suppressed by perturbative couplings and is enhanced by the sum over all partons, particularly the gluon.

Subsequent to formation, the $\mathrm{BH}$ proceeds to decay dominantly through radiation of standard SM particles on the 3-brane [286]. This occurs because the SM particles live on the brane, so that the relevant phase space for $\mathrm{BH}$ decay into these fields

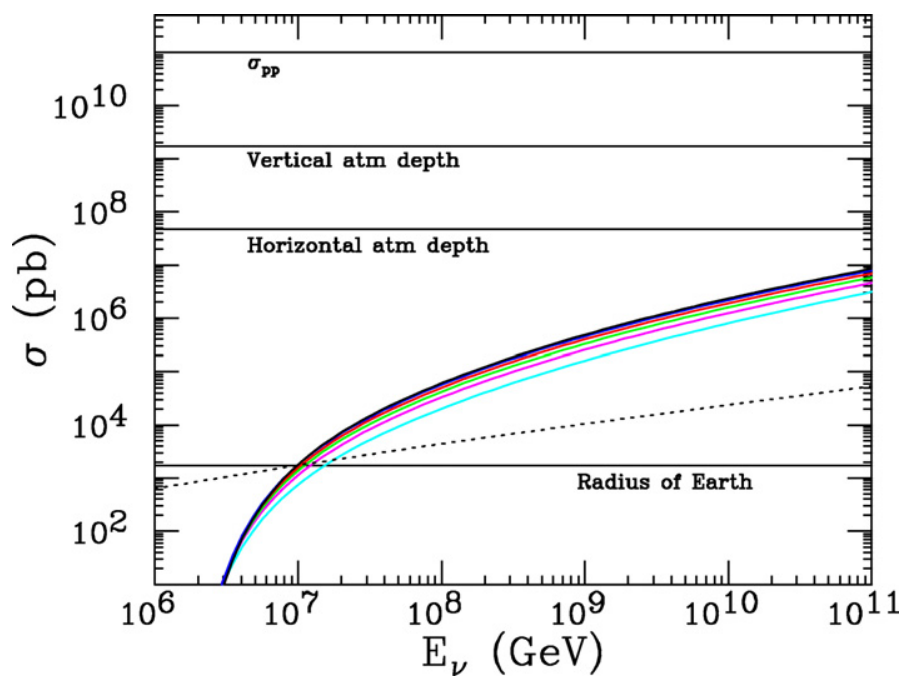

Fig. 22. Lower bounds on $\mathrm{BH}$ production cross sections for $n=2, \ldots, 7$ from below, assuming $M_{D}=1 \mathrm{TeV}$ and $x_{\min }=1$. Energy loss has been included according to Eq. (85). The SM cross section $\sigma_{v N}^{\mathrm{CC}}$, as given in Eq. (75), is indicated by the dotted line. The typical range of $p p$ interactions, as well as cross sections required for shower triggering in different characteristic targets are also shown for comparison. This figure is courtesy of Jonathan Feng. 
is governed by the four-dimensional projection of the horizon area of the $(4+n)$-dimensional $\mathrm{BH}$, and by the Hawking temperature which is common to bulk and brane. Modulo some grey body factors [287-289], the dominance of the SM radiation is a result of the much larger number of degrees of freedom.

The choice of a lower limit of integration in Eq. (85) requires additional explanation. This limit determines the minimum mass for $\mathrm{BHs}$ that will be included in the calculation. The semiclassical description outlined above is only reliable when the energy of the emitted particle is small compared to the BH mass, because it is only under this condition that both the gravitational field of the brane and the back reaction of the metric during the emission process can be safely neglected [290]. Since the total number of particles emitted by the $\mathrm{BH}$ is roughly equal to its entropy

$$
S=\frac{4 \pi M_{\mathrm{BH}} r_{s}\left(M_{\mathrm{BH}}\right)}{n+2},
$$

the criterion we employ is to assume that the $\mathrm{BH}$ has an entropy $S \gg 1$. For $x_{\text {min }} \equiv M_{\mathrm{BH} \text {, } \min } / M_{D}>3$ and $n \geqslant 5$, one finds $S>10$, so that most of the decay process can be well described within the semiclassical approximation. Moreover, the string cross section derived [291] from the Virasoro-Shapiro amplitude is expected to be considerably larger than that given in Eq. (82), and so it may be reasonable even to take $x_{\min }$ as low as 1 , for which $S \gtrsim 3$ [292].

Although the $\mathrm{BH}$ production cross section, $\mathcal{O}\left(M_{\mathrm{EW}}^{-1}\right)$, is about five orders of magnitude smaller than QCD cross sections, $\mathcal{O}\left(\Lambda_{\mathrm{QCD}}^{-1}\right)$, one of the most startling predictions of TeV-scale gravity theories is that at the $\mathrm{LHC}, \mathrm{BH}$ events could be filtered out of the QCD background, both in $p p$ [274,275] (see also [293-298]) as well as in $\mathrm{PbPb}$ [299] collisions. At energies of interest, however, the cosmic ray luminosity, $L \sim 10^{-24} \mathrm{~cm}^{-2} \mathrm{~s}^{-1}$, is about 50 orders of magnitude smaller than the LHC luminosity, thus making it futile to hunt for BHs in baryonic cosmic rays. On the other hand, as can be seen in Fig. 22, although greatly reduced by the cross section for BH production, neutrino interaction lengths are still far larger than the Earth's atmospheric depth. Neutrinos therefore would produce BHs with roughly equal probability at any point in the atmosphere. As a result, the light descendants of the $\mathrm{BH}$ may initiate low-altitude, quasi-horizontal showers at rates significantly higher than SM predictions. Because of these considerations the atmosphere provides a buffer against contamination by mismeasured baryons (for which the electromagnetic channel is filtered out) allowing a good characterization of BH-induced showers when $S \gg 1$ [300-304]. ${ }^{13}$ Furthermore, a similar technique to that employed in discriminating between photon and hadron showers can be applied to isolate $\mathrm{BH}$ mediated showers from neutrino SM events [308]. Specifically, if an anomalously large quasi-horizontal deep shower rate is found, it may be ascribed to either an enhancement of the incoming neutrino flux, or an enhancement in the neutrino-nucleon cross section. However, these two possibilities may be distinguished by separately binning events which arrive at very small angles to the horizontal, the so-called "Earth-skimming",

\footnotetext{
13 Additionally, neutrinos that traverse the atmosphere unscathed may produce BHs through interactions in the ice or water and be detected by neutrino telescopes [305-307].
} 
events. An enhanced flux will increase both quasi-horizontal and Earth-skimming event rates, whereas a large $\mathrm{BH}$ cross section suppresses the latter, because the hadronic decay products of $\mathrm{BH}$ evaporation do not escape the Earth's crust.

In summary, the signal for ultra high energy neutrinos is quasi-horizontal giant air showers initiated deep in the atmosphere: showers with large electromagnetic components, curved fronts, and signals well spread over time. These shower characteristics are easily differentiated from EAS initiated by baryons or photons. The low target density for neutrino interactions provided by the atmosphere must be compensated by monitoring large areas at the Earth's surface. In particular, the PAO will have an acceptance exceeding $1 \mathrm{~km}^{3}$ of water for $E_{v} \gtrsim 10^{8} \mathrm{GeV}$, and thus will be able to search for extraterrestrial sources of ultra high energy neutrinos. Moreover, this observatory holds great promise for probing physics beyond the SM. An optimist might even imagine the discovery of microscopic BHs, the telltale signature of the universe's unseen dimensions.

\section{EAS in a nutshell}

In this article, we have reviewed the general properties and techniques for modelling air showers initiated by ultra high energy particles interacting in the Earth's atmosphere.

The incidence of a single high energy particle on the upper atmosphere gives rise to a roughly conical cascade of particles which reaches the Earth in the form of a giant "saucer" traveling at nearly the speed of light. The number of secondaries in the cascade readily increases through subsequent generations of particle interactions. Because of the prompt decay of neutral pions, about $30 \%$ of the energy in each generation is transferred to an electromagnetic cascade. Roughly speaking, at $10^{11} \mathrm{GeV}$, baryons and charged pions have interaction lengths of the order of $40 \mathrm{~g} / \mathrm{cm}^{2}$, increasing to about $60 \mathrm{~g} / \mathrm{cm}^{2}$ at $10^{7} \mathrm{GeV}$. Additionally, below $10^{10} \mathrm{GeV}$, photons, electrons, and positrons have mean interaction lengths of $37 \mathrm{~g} / \mathrm{cm}^{2}$, whereas above this critical energy the competing LPM and geomagnetic effects lead to interaction lengths between 45 and $60 \mathrm{~g} / \mathrm{cm}^{2}$. Altogether, the atmosphere acts as a natural colorimeter with variable density, providing a vertical thickness of 26 radiation lengths and about 15 interaction lengths. Amusingly, this is not too different from the number of radiation and interaction lengths at the LHC detectors. ${ }^{14}$

The number of muons does not increase linearly with energy, because of the previously mentioned pionization process: at higher energy more generations are required to cool the pions to the point where they are likely to decay before interaction. Production of extra generations results in a larger fraction of the energy being lost to the electromagnetic cascade, and hence a smaller fraction of the original energy being delivered to the $\pi^{ \pm}$. Ultimately, about $90 \%$ of the primary particle's

\footnotetext{
${ }^{14}$ For example, the CMS electromagnetic calorimeter is $\gtrsim 25$ radiation lengths deep, and the hadron calorimeter constitutes 11 interaction lengths.
} 
energy is dissipated in the electromagnetic cascade. The remaining energy is carried by hadrons, as well as muons and neutrinos produced in $\pi^{ \pm}$decays.

By the time they reach the ground, relatively vertical showers have evolved fronts with a curvature radius of a few $\mathrm{km}$, and far from the shower core their constituent particles are well spread over time, typically of the order of a few microseconds. For such a shower both the muon component and a large portion of the electromagnetic component survive to reach the ground, and their lateral distributions can be accurately parametrized. Although the lateral distribution function depends on the experiment, surface measurements of both $\gamma$ - and baryon-induced showers can be fitted with NKG-like formulae. From this distribution the primary energy can be determined.

For inclined showers the electromagnetic component is absorbed long before reaching the ground, as it has passed through the equivalent of several vertical atmospheres: 2 at a zenith angle of $60^{\circ}, 3$ at $70^{\circ}$, and 6 at $80^{\circ}$. In these showers, only high energy muons created in the first few generations of particles survive past two equivalent vertical atmospheres. The rate of energy attenuation for muons is much smaller than it is for electrons, thus the shape of the resulting shower front is very flat (with curvature radius above $100 \mathrm{~km}$ ), and its time extension is very short (less than $50 \mathrm{~ns}$ ). The damping of the electromagnetic component of the shower provides a means to search for both photon and neutrino primaries. In particular quasi-horizontal showers with electromagnetic components at the ground would suggest a deeply penetrating primary, such as the elusive ultra high energy neutrino.

As we have seen, the chief uncertainty in shower modelling arises from lack of definitive knowledge about the nature of hadronic interactions. This is because the center-of-mass energies involved in cosmic ray collisions are orders of magnitude beyond that achievable in present and foreseeable future experiments. Moreover, man-made accelerators are designed to probe QCD physics in the high transverse momentum region, and air shower physics is driven by interactions in the very forward direction. The analysis of extensive air showers then requires the extrapolation of hadronic interaction models more than two orders of magnitude in center-of-mass energy beyond the highest accelerator energies $(\sqrt{s}=1.8 \mathrm{TeV})$ to date. In fact, the required extrapolation is much greater than this because air showers involve nuclei as well as single hadrons both as targets and projectiles. Efforts towards improving our understanding of soft and semi-hard processes are clearly required.

The muon content of the shower tail is quite sensitive to the unknown details of hadronic physics at ultra high energies. This implies that attempts to extract composition information from measurements of muon content at ground level tend to be systematics dominated. There are, however, complimentary methods for uncovering the primary species which are less dependent on knowledge of the hadronic physics. One well-established method involves using fluorescence telescopes to determine the energy dependence of the depth of shower maximum, the so-called elongation rate, which is sensitive to the evolution of the primary composition with energy. Unfortunately, fluorescence detection has its own set of systematic uncertainties associated with the knowledge of atmospheric properties as a function of time. Future hybrid experiments, such as the PAO, will record events with simultaneous observation of 
particles reaching the ground and the shower profile in the atmosphere, and thus provide a new arsenal of data for controlling the systematic errors. Furthermore, the giant aperture of the array will generate an extensive air shower sample of unprecedented size, ushering in a golden age of cosmic ray physics.

\section{Acknowledgments}

We thank Máximo Ave, Jim Cronin, Luis Epele, Jonathan Feng, Haim Goldberg, Francis Halzen, Dieter Heck, Johannes Knapp, Mónica Manceñido, Markus Risse, Markus Roth, Sergio Sciutto, Al Shapere, Alan Watson, Tom Weiler, and Tokonatsu Yamamoto for fruitful collaborations. We also acknowledge Albert de Roeck and our colleagues of the AUGER, L3, and D0 collaborations for many illuminating discussions. We are indebted to several collaborators mentioned above as well as Felix Aharonian, Greg Archbold, Karsten Eggert, Max Klein, Kenji Shinozaki, Hristofor Vankov, Enrique Zas, and the HiRes and AGASA collaborations for allowing us to use various figures from their papers in this review. This work has been supported, in part, by the US National Science Foundation (under Grant No. PHY0140407) and IFLP-CONICET Argentina.

\section{References}

[1] J. Linsley, Phys. Rev. Lett. 10 (1963) 146.

[2] D.J. Bird, et al., Astrophys. J. 441 (1995) 144.

[3] P. Sokolsky, P. Sommers, B.R. Dawson, Phys. Rep. 217 (1992) 225.

[4] S. Yoshida, H. Dai, J. Phys. G 24 (1998) 905. Available from: <arxiv:astro-ph/9802294>.

[5] J.W. Cronin, Rev. Mod. Phys. 71 (1999) S165.

[6] M. Nagano, A.A. Watson, Rev. Mod. Phys. 72 (2000) 689.

[7] X. Bertou, M. Boratav, A. Letessier-Selvon, Int. J. Mod. Phys. A 15 (2000) 2181. Available from: $<$ arxiv:astro-ph/0001516>

[8] A.A. Watson, Phys. Rept. 333 (2000) 309.

[9] J.W. Cronin. Available from: <arxiv:astro-ph/0402487>.

[10] A.M. Hillas, Ann. Rev. Astron. Astrophys. 22 (1984) 425.

[11] P.L. Biermann, J. Phys. G 23 (1997) 1.

[12] P. Bhattacharjee, G. Sigl, Phys. Rep. 327 (2000) 109. Available from: <arxiv:astro-ph/9811011>.

[13] V.A. Kuzmin, I.I. Tkachev, Phys. Rep. 320 (1999) 199. Available from: <arxiv:hep-ph/9903542>.

[14] A.V. Olinto, Phys. Rep. 333 (2000) 329. Available from: <arxiv:astro-ph/0002006>.

[15] L. Anchordoqui, T. Paul, S. Reucroft, J. Swain, Int. J. Mod. Phys. A 18 (2003) 2229. Available from: <arxiv:hep-ph/0206072>.

[16] G. Sigl, Ann. Phys. 303 (2003) 117. Available from: <arxiv:astro-ph/0210049>.

[17] F.W. Stecker, J. Phys. G 29 (2003) R47. Available from: <arxiv:astro-ph/0309027>.

[18] D.F. Torres, L.A. Anchordoqui. Available from: <arxiv:astro-ph/0402371>.

[19] M. Nagano, et al., J. Phys. G 18 (1992) 423.

[20] A.N. Bunner, PhD Thesis, Cornell University, Ithaca, NY, 1964.

[21] M. Nagano, K. Kobayakawa, N. Sakaki, K. Ando. Available from: <arxiv:astro-ph/0406474>.

[22] B. Keilhauer, J. Blumer, R. Engel, H.O. Klages, M. Risse. Available from: <arxiv:astro-ph/ 0405048>.

[23] J. Linsley, L. Scarsi, B. Rossi, Phys. Rev. Lett. 6 (1961) 485. 
[24] M.A. Lawrence, R.J. Reid, A.A. Watson, J. Phys. G 17 (1991) 733.

[25] G.B. Khristiansen, in: Proceedings of 19th International Cosmic Ray Conference, La Jolla, vol. 9, p. 487, 1985.

[26] M.M. Winn, J. Ulrichs, L.S. Peak, C.B. Mccusker, L. Horton, J. Phys. G 12 (1986) 653.

[27] N. Chiba, et al., Nucl. Instrum. Methods A 311 (1992) 338.

[28] H. Ohoka et al. (AGASA Collaboration), Nucl. Instrum. Methods A 385 (1997) 268.

[29] R.M. Baltrusaitis, et al., Nucl. Instrum. Methods A 240 (1985) 410.

[30] R.M. Baltrusaitis, et al., Nucl. Instrum. Methods A 264 (1988) 87.

[31] T. Abu-Zayyad, et al., Nucl. Instrum. Methods A 450 (2000) 253.

[32] J. Abraham et al. (AUGER Collaboration), Nucl. Instrum. Methods A 523 (2004) 50.

[33] K.S. Capelle, J.W. Cronin, G. Parente, E. Zas, Astropart. Phys. 8 (1998) 321. Available from: $<$ astro-ph/9801313>.

[34] O. Catalano, Nuovo Cim. C 24 (2001) 445.

[35] L. Scarsi, Nuovo Cim. C 24 (2001) 471.

[36] M. Takeda, et al., Phys. Rev. Lett. 81 (1998) 1163. Available from: <arxiv:astro-ph/9807193>.

[37] M. Takeda, et al., Astropart. Phys. 19 (2003) 447. Available from: <arxiv:astro-ph/0209422>.

[38] K. Greisen, Phys. Rev. Lett. 16 (1966) 748.

[39] G.T. Zatsepin, V.A. Kuzmin, JETP Lett. 4 (1966) 78. [Pisma Zh. Eksp. Teor. Fiz. 4 (1966) 114].

[40] T. Abu-Zayyad et al. (HiRes Collaboration). Available from: <arxiv:astro-ph/0208301>.

[41] D.J. Bird et al. (HiRes Collaboration), Astrophys. J. 424 (1994) 491.

[42] D. De Marco, P. Blasi, A.V. Olinto, Astropart. Phys. 20 (2003) 53. Available from: <arxiv:astro-ph/ 0301497>.

[43] L.A. Anchordoqui, C. Hojvat, T.P. McCauley, T.C. Paul, S. Reucroft, J.D. Swain, A. Widom, Phys. Rev. D 68 (2003) 083004. Available from: <arxiv:astro-ph/0305158>.

[44] R. Abbasi et al. (HiRes Collaboration). Available from: <arxiv:astro-ph/0309457>.

[45] N. Hayashida, et al., Phys. Rev. Lett. 77 (1996) 1000.

[46] N. Hayashida et al. (AGASA Collaboration). Available from: <arxiv:astro-ph/0008102>.

[47] R.U. Abbasi et al. (HiRes Collaboration). Available from: <arxiv:astro-ph/0404137>.

[48] R.U. Abbasi et al. (HiRes Collaboration). Available from: <arxiv:astro-ph/0404366>.

[49] C.B. Finley, S. Westerhoff, Astropart. Phys. 21 (2004) 359. Available from: <arxiv:astro-ph/ 0309159>.

[50] A. Capella, U. Sukhatme, C.I. Tan, J. Tran Thanh Van, Phys. Rep. 236 (1994) 225.

[51] E. Predazzi. Available from: <arxiv:hep-ph/9809454>.

[52] D. Cline, F. Halzen, J. Luthe, Phys. Rev. Lett. 31 (1973) 491.

[53] S.D. Ellis, M.B. Kislinger, Phys. Rev. D 9 (1974) 2027.

[54] F. Halzen, Nucl. Phys. B 92 (1975) 404.

[55] T.K. Gaisser, F. Halzen, Phys. Rev. Lett. 54 (1985) 1754.

[56] G. Pancheri, C. Rubbia, Nucl. Phys. A 418 (1984) 117C.

[57] G. Pancheri, Y. Srivastava, Phys. Lett. B 159 (1985) 69.

[58] G. Pancheri, Y.N. Srivastava, Phys. Lett. B 182 (1986) 199.

[59] C. Albajar et al. (UA1 Collaboration), Nucl. Phys. B 309 (1988) 405.

[60] C. Adloff et al. (H1 Collaboration), Eur. Phys. J. C 21 (2001) 33. Available from: <arxiv:hep-ex/ 0012053>.

[61] G. Ingelman, P.E. Schlein, Phys. Lett. B 152 (1985) 256.

[62] L. Alvero, J.C. Collins, J. Terron, J.J. Whitmore, Phys. Rev. D 59 (1999) 074022. Available from: $<$ arxiv:hep-ph/9805268>.

[63] V.N. Gribov, L.N. Lipatov, Yad. Fiz. 15 (1972) 1218. Sov. J. Nucl. Phys. 15 (1972) 675.

[64] V.N. Gribov, L.N. Lipatov, Yad. Fiz. 15 (1972) 781. Sov. J. Nucl. Phys. 15 (1972) 438.

[65] Y.L. Dokshitzer, Sov. Phys. JETP 46 (1977) 641. Zh. Eksp. Teor. Fiz. 73 (1977) 1216.

[66] G. Altarelli, G. Parisi, Nucl. Phys. B 126 (1977) 298.

[67] H. Abramowicz, A. Caldwell, Rev. Mod. Phys. 71 (1999) 1275. Available from: <arxiv:hep-ex/ 9903037>.

[68] R. Engel, Nucl. Phys. Proc. Suppl. 122 (2003) 40. 
[69] L.V. Gribov, E.M. Levin, M.G. Ryskin, Phys. Rep. 100 (1983) 1.

[70] J. Kwiecinski, A.D. Martin, Phys. Rev. D 43 (1991) 1560.

[71] R.J. Glauber, G. Matthiae, Nucl. Phys. B 21 (1970) 135.

[72] P. L'Heureux, B. Margolis, P. Valin, Phys. Rev. D 32 (1985) 1681.

[73] L. Durand, H. Pi, Phys. Rev. Lett. 58 (1987) 303.

[74] L. Durand, H. Pi, Phys. Rev. D 38 (1988) 78.

[75] M.M. Block, R.N. Cahn, Rev. Mod. Phys. 57 (1985) 563.

[76] K.A. Ter-Martirosyan, Phys. Lett. B 44 (1973) 377.

[77] V.A. Abramovsky, V.N. Gribov, O.V. Kancheli, Yad. Fiz. 18 (1973) 595. Sov. J. Nucl. Phys. 18 (1974) 308.

[78] T.K. Gaisser, T. Stanev, Phys. Lett. B 219 (1989) 375.

[79] R.S. Fletcher, T.K. Gaisser, P. Lipari, T. Stanev, Phys. Rev. D 50 (1994) 5710.

[80] N.N. Kalmykov, S.S. Ostapchenko, A.I. Pavlov, Nucl. Phys. Proc. Suppl. B 52 (1997) 17.

[81] R. Engel, T.K. Gaisser, T. Stanev, in: Proceedings of the 27th International Cosmic Ray Conference, Hamburg, p. 431, 2001.

[82] F. Abe et al. (CDF Collaboration), Phys. Rev. D 56 (1997) 3811.

[83] G. Calucci, D. Treleani, Phys. Rev. D 60 (1999) 054023. Available from: <arxiv:hep-ph/9902479>.

[84] L.A. Anchordoqui, M.T. Dova, L.N. Epele, S.J. Sciutto, Phys. Rev. D 59 (1999) 094003. Available from: <arxiv:hep-ph/9810384>.

[85] L.A. Anchordoqui, M.T. Dova, S.J. Sciutto, in: Proceedings of the 26th International Cosmic Ray Conference, Salt Lake City, vol. 1, p. 147, 1999. Available from: <arxiv:hep-ph/9905248>.

[86] J. Alvarez-Muniz, R. Engel, T.K. Gaisser, J.A. Ortiz, T. Stanev, Phys. Rev. D 66 (2002) 033011. Available from: <arxiv:astro-ph/0205302>.

[87] R. Engel, H. Rebel, Acta Phys. Polon. B 35 (2004) 321.

[88] M. Froissart, Phys. Rev. 123 (1961) 1053.

[89] U. Amaldi, K.R. Schubert, Nucl. Phys. B 166 (1980) 301.

[90] M.M. Block, F. Halzen, T. Stanev, Phys. Rev. D 62 (2000) 077501. Available from: <arxiv:hep-ph/ $0004232>$.

[91] M. Honda, et al., Phys. Rev. Lett. 70 (1993) 525.

[92] R.M. Baltrusaitis, et al., Phys. Rev. Lett. 52 (1984) 1380.

[93] T.K. Gaisser, U. Sukhatme, G.B. Yodh, Phys. Rev. D 36 (1987) 1350.

[94] B.Z. Kopeliovich, N.N. Nikolaev, I.K. Potashnikova, Phys. Rev. D 39 (1989) 769.

[95] N.N. Nikolaev, Phys. Rev. D 48 (1993) 1904. Available from: <arxiv:hep-ph/9304283>.

[96] M.M. Block, F. Halzen, T. Stanev, Phys. Rev. Lett. 83 (1999) 4926. Available from: <arxiv:hep-ph/ 9908222>.

[97] J. Ranft, Phys. Rev. D 51 (1995) 64.

[98] G. Bossard, et al., Phys. Rev. D 63 (2001) 054030. Available from: <arxiv:hep-ph/0009119>.

[99] R. Engel, T.K. Gaisser, T. Stanev, P. Lipari, in: Proceedings of the 26th International Cosmic Ray Conference, Salt Lake City, vol. 1, p. 415, 1999.

[100] D. Heck, in: Proceedings of the 27th International Cosmic Ray Conference, Hamburg, p. 233, 2001.

[101] S. Roesler, R. Engel, J. Ranft. Available from: <arxiv:hep-ph/0012252>.

[102] T. Sjostrand, Int. J. Mod. Phys. A 3 (1988) 751.

[103] J. Engel, T.K. Gaisser, T. Stanev, P. Lipari, Phys. Rev. D 46 (1992) 5013.

[104] A.B. Kaidalov, K.A. Ter-Martirosian, Y.M. Shabelski, Sov. J. Nucl. Phys. 43 (1986) 822. Yad. Fiz. 43 (1986) 1282.

[105] J.E. Elias, W. Busza, C. Halliwell, D. Luckey, P. Swartz, L. Votta, C. Young, Phys. Rev. D 22 (1980) 13.

[106] A. Bialas, M. Bleszynski, W. Czyz, Nucl. Phys. B 111 (1976) 461.

[107] X.N. Wang, M. Gyulassy, Phys. Rev. Lett. 86 (2001) 3496. Available from: <arxiv:nucl-th/ 0008014>.

[108] K.J. Eskola, K. Kajantie, K. Tuominen, Phys. Lett. B 497 (2001) 39. Available from: <arxiv:hep-ph/ 0009246>. 
[109] I.G. Bearden et al. (BRAHMS Collaboration), Phys. Rev. Lett. 88 (2002) 202301. Available from: $<$ arxiv:nucl-ex/0112001>.

[110] K. Adcox et al. (PHENIX Collaboration), Phys. Rev. Lett. 86 (2001) 3500. Available from: $<$ arxiv:nucl-ex/0012008>.

[111] S.R. Klein, Nucl. Phys. Proc. Suppl. 122 (2003) 76. Available from: <arxiv:astro-ph/0211018>.

[112] J. Dias de Deus, R. Ugoccioni, Phys. Lett. B 491 (2000) 253. Available from: <arxiv:hep-ph/ 0008086>

[113] J. Dias de Deus, R. Ugoccioni, Phys. Lett. B 494 (2000) 53. Available from: <arxiv:hep-ph/ 0009288>.

[114] M.A. Braun, C. Pajares, J. Ranft, Int. J. Mod. Phys. A 14 (1999) 2689. Available from: <arxiv:hep$\mathrm{ph} / 9707363>$.

[115] M.A. Braun, C. Pajares, Eur. Phys. J. C 16 (2000) 349. Available from: <arxiv:hep-ph/9907332>.

[116] M.A. Braun, F. Del Moral, C. Pajares, Phys. Rev. C 65 (2002) 024907. Available from: <arxiv:hep$\mathrm{ph} / 0105263>$.

[117] B.B. Back et al. (PHOBOS Collaboration). Available from: <arxiv:nucl-ex/0311009>.

[118] F.W. Bopp, J. Ranft, R. Engel, S. Roesler. Available from: <arxiv:hep-ph/0403084>.

[119] T. Antoni et al. (KASCADE Collaboration), J. Phys. G 27 (2001) 1785. Available from: $<$ arxiv:astro-ph/0106494>.

[120] L.R. Evans, CERN-LHC-PROJECT-REPORT-303. Invited talk at IEEE Particle Accelerator Conference (PAC 99), New York, New York, 29 Mar-2 Apr 1999.

[121] K. Eggert, Nucl. Phys. Proc. Suppl. 122 (2003) 447.

[122] TOTEM Collaboration, Total cross-section, elastic scattering and difraction dissociation at LHC, Technical Proposal, CERN/LHCC 99-7, 1999.

[123] TOTEM Technical Design Report, CERN-LHCC-2004-002.

[124] G. Matthiae, Nucl. Phys. Proc. Suppl. A 99 (2001) 281.

[125] P. Lipari, Nucl. Phys. Proc. Suppl. 122 (2003) 133. Available from: <arxiv:astro-ph/0301196>.

[126] C. Augier et al. (UA4/2 Collaboration), Phys. Lett. B 315 (1993) 503.

[127] M. Rijssenbeek, Nucl. Phys. Proc. Suppl. 122 (2003) 459.

[128] L.W. Jones, Nucl. Phys. Proc. Suppl. 122 (2003) 433.

[129] R. Engel, Nucl. Phys. Proc. Suppl. 122 (2003) 437. Available from: <arxiv:hep-ph/0212340>.

[130] G. Schatz, Nucl. Phys. Proc. Suppl. 122 (2003) 462.

[131] D. Heck, Nucl. Phys. Proc. Suppl. 122 (2003) 451.

[132] H.M.J. Barbosa, F. Catalani, J.A. Chinellato, C. Dobrigkeit. Available from: <arxiv:astro-ph/ 0310234>.

[133] T.J.L. Mccomb, R.J. Protheroe, K.E. Turver, J. Phys. G 5 (1979) 1613.

[134] B. Rossi, K. Greisen, Rev. Mod. Phys. 13 (1941) 240.

[135] T.K. Gaisser, Cosmic Rays and Particle Physics, Cambridge University Press, Cambridge, 1990.

[136] H. Bethe, W. Heitler, Proc. Roy. Soc. Lond. A 146 (1934) 83.

[137] S. Eidelman et al. (Particle Data Group Collaboration), Phys. Lett. B 592 (2004) 1.

[138] B. Rossi, High Energy Particles, Prentice-Hall, Englewood Cliffs, NY, 1952.

[139] R.C. Weast, CRC Handbook of Chemistry and Physics, CRC Press, Boca Raton, FL, USA, 1981.

[140] Y.S. Tsai, Rev. Mod. Phys. 46 (1974) 815 [Erratum Rev. Mod. Phys. 49 (1977) 421].

[141] L.D. Landau, I. Pomeranchuk, Dokl. Akad. Nauk Ser. Fiz. 92 (1953) 535.

[142] A.B. Migdal, Phys. Rev. 103 (1956) 1811.

[143] T. Stanev, C. Vankov, R.E. Streitmatter, R.W. Ellsworth, T. Bowen, Phys. Rev. D 25 (1982) 1291.

[144] P.L. Anthony et al. (SLAC-E-146 Collaboration), Phys. Rev. D 56 (1997) 1373. Available from: $<$ arxiv:hep-ex/9703016>

[145] S. Klein, Rev. Mod. Phys. 71 (1999) 1501. Available from: <arxiv:hep-ph/9802442>.

[146] J. Alvarez-Muniz, E. Zas, Phys. Lett. B 411 (1997) 218. Available from: <arxiv:astro-ph/9706064>.

[147] J. Alvarez-Muniz, E. Zas, Phys. Lett. B 434 (1998) 396. Available from: <arxiv:astro-ph/9806098>.

[148] A.N. Cillis, H. Fanchiotti, C.A. Garcia Canal, S.J. Sciutto, Phys. Rev. D 59 (1999) 113012. Available from: <arxiv:astro-ph/9809334>.

[149] J.N. Capdevielle, C. Le Gall, K.N. Sanosian, Astropart. Phys. 13 (2000) 259. 
[150] A.V. Plyasheshnikov, F.A. Aharonian, J. Phys. G 28 (2002) 267. Available from: <arxiv:astro-ph/ 0107592>.

[151] S.J. Sciutto. Available from: <arxiv:astro-ph/9911331>.

[152] T. Erber, Rev. Mod. Phys. 38 (1966) 626.

[153] B. Mcbreen, C.J. Lambert, Phys. Rev. D 24 (1981) 2536.

[154] X. Bertou, P. Billoir, S. Dagoret-Campagne, Astropart. Phys. 14 (2000) 121.

[155] H.P. Vankov, N. Inoue, K. Shinozaki, Phys. Rev. D 67 (2003) 043002. Available from: <arxiv:astro$\mathrm{ph} / 0211051>$.

[156] P. Homola et al. Available from: <arxiv:astro-ph/0311442>.

[157] M. Risse, P. Homola, D. Gora, J. Pekala, B. Wilczynska, H. Wilczynski. Available from: $<$ arxiv:astro-ph/0401629>.

[158] F. Halzen, R.A. Vazquez, T. Stanev, H.P. Vankov, Astropart. Phys. 3 (1995) 151.

[159] L.A. Anchordoqui, M. Kirasirova, T.P. McCauley, S. Reucroft, J.D. Swain, Phys. Lett. B 492 (2000) 237. Available from: <arxiv:astro-ph/0007403>.

[160] W. Heitler, The Quantum Theory of Radiation, second ed., Oxford University Press, London, 1944.

[161] D. Heck, G. Schatz, T. Thouw, J. Knapp, J.N. Capdevielle, FZKA-6019, 1998.

[162] J. Knapp, D. Heck, S.J. Sciutto, M.T. Dova, M. Risse, Astropart. Phys. 19 (2003) 77. Available from: <arxiv:astro-ph/0206414>.

[163] H.J. Drescher, G.R. Farrar, Phys. Rev. D 67 (2003) 116001. Available from: <arxiv:astro-ph/ 0212018>.

[164] H.J. Drescher, G.R. Farrar, Astropart. Phys. 19 (2003) 235. Available from: <arxiv:hep-ph/ 0206112>.

[165] K. Kamata, J. Nishimura, Progr. Theor. Phys. Suppl. 6 (1958) 93.

[166] K. Greisen, Ann. Rev. Nucl. Sci. 10 (1960) 63.

[167] M.T. Dova, L.N. Epele, A.G. Mariazzi, Astropart. Phys. 18 (2003) 351. Available from: $<$ arxiv:astro-ph/0110237>.

[168] J. Linsley, in: Proceedings of the 13th International Cosmic Ray Conference, Denver, vol. 5, p. 3212, 1973.

[169] C. Aguirre et al, in: Proceedings of the 13th International Cosmic Ray Conference, Denver, vol. 4, p. 2592, 1973.

[170] N.A. Porter, in: Proceedings of the 13th International Cosmic Ray Conference, Denver, vol. 5, p. 3657, 1973.

[171] S. Kawaguchi, K. Suga, H. Sakuyama, in: Proceedings of the 14th International Cosmic Ray Conference, Munich, vol. 8, p. 2826, 1975.

[172] M. Nagano, et al., J. Phys. Soc. Japan 53 (1984) 1667.

[173] S. Yoshida, et al., J. Phys. G 20 (1994) 651.

[174] A.V. Glushkov et al, in: Proceedings of the 25th International Cosmic Ray Conference, Durban, vol. 6, p. 233, 1997.

[175] R.N. Coy, et al., Astropart. Phys. 6 (1997) 263.

[176] M.F. Bourdeau, J.N. Capdevielle, J. Procureur, J. Phys. G 6 (1980) 901.

[177] A.V. Plyasheshnikov, A.A. Lagutin, V.V. Uchaikin, in: Proceedings of the 16th International Cosmic Ray Conference, Kyoto, vol. 7, p. 1, 1979.

[178] M. Roth (AUGER Collaboration). Available from: <arxiv:astro-ph/0308392>.

[179] X. Bertou, P. Billoir, Auger Note (GAP-2000-017). Available from: <www.auger.org/admin>.

[180] M.T. Dova, L.N. Epele, A. Mariazzi, Nuovo Cim. C 24 (2001) 745.

[181] A. Cillis, S.J. Sciutto, J. Phys. G 26 (2000) 309.

[182] M. Ave, R.A. Vazquez, E. Zas, J.A. Hinton, A.A. Watson, Astropart. Phys. 14 (2000) 109. Available from: <arxiv:astro-ph/0003011>.

[183] L. Anchordoqui, H. Goldberg, Phys. Lett. B 583 (2004) 213. Available from: <arxiv:hep-ph/ 0310054>.

[184] A.N. Cillis, S.J. Sciutto, Phys. Rev. D 64 (2001) 013010. Available from: <arxiv:astro-ph/0010488>.

[185] C. Adloff et al. (H1 Collaboration), Nucl. Phys. B 497 (1997) 3. Available from: <arxiv:hep-ex/ 9703012>. 
[186] M. Derrick et al. (ZEUS Collaboration), Phys. Lett. B 293 (1992) 465.

[187] S.N. Vernov, G.B. Khristiansen, A.T. Abrasimov, V.B. Atrashkevitch, I.F. Beljaeva, G.V. Kulikov, K.V. Mandritskaya, K.B. Solovjeva, B.A. Khrenov, Can. J. Phys. 46 (1968) s197.

[188] A.M. Hillas, et al., Acta Phys. Acad. Sci. Hung. 29 (Suppl. 3) (1970) 533.

[189] R.G. Brownlee, et al., Acta Phys. Acad. Sci. Hung. 29 (Suppl. 3) (1970) 651.

[190] T. Antoni et al. (KASCADE Collaboration), Astropart. Phys. 14 (2001) 245. Available from: <arxiv:astro-ph/0004233>.

[191] S. Alessio, H. Bilokon, B. D’Ettorre Piazzoli, G. Mannocchi, P. Picchi, K. Sitte, Nuovo Cim. C 3 (1980) 573.

[192] G. Bosia, G. Navarra, O. Saavedra, E. Bohm, Nuovo Cim. C 3 (1980) 215.

[193] L. Bergamasco, M. Castagnoli, M. Dardo, B. D’Ettore Piazzoli, G. Mannochi, P. Pichi, R. Visentin, K. Sitte, Nuovo Cim. A 34 (1976) 613.

[194] M. Ave, R.A. Vazquez, E. Zas, Astropart. Phys. 14 (2000) 91. Available from: <arxiv:astro-ph/ $0011490>$.

[195] A.M. Hillas, J.D. Hollows, H.W. Hunter, D.J. Marsden, in: Proceedings of the 12th International Cosmic Ray Conference, Hobart, vol. 3, p. 1007, 1971.

[196] A.M. Hillas, D.J. Marsden, J.D. Hollows, H.W. Hunter, in: Proceedings of the 12th International Cosmic Ray Conference, Hobart, vol. 3, p. 1001, 1971.

[197] M. Ave, J.A. Hinton, R.A. Vazquez, A.A. Watson, E. Zas, Phys. Rev. D 65 (2002) 063007. Available from: <arxiv:astro-ph/0110613>.

[198] J. Alvarez-Muniz, R. Engel, T.K. Gaisser, J.A. Ortiz, T. Stanev, Phys. Rev. D 66 (2002) 123004. Available from: <arxiv:astro-ph/0209117>.

[199] K. Shinozaki et al. (AGASA Collaboration), in: Proceedings of the 28th International Cosmic Ray Conference, Tsukuba, p. 401, 2003.

[200] K. Shinozaki, et al., Astrophys. J. 571 (2002) L117.

[201] M. Ave, J.A. Hinton, R.A. Vazquez, A.A. Watson, E. Zas, Phys. Rev. Lett. 85 (2000) 2244. Available from: <arxiv:astro-ph/0007386>.

[202] M. Ave, J.A. Hinton, R.A. Vazquez, A.A. Watson, E. Zas, Astropart. Phys. 18 (2003) 367. Available from: <arxiv:astro-ph/0112071>.

[203] M. Ave, J.A. Hinton, R.A. Vazquez, A.A. Watson, E. Zas, Phys. Rev. D 67 (2003) 043005. Available from: <arxiv:astro-ph/0208228>.

[204] T.K. Gaisser, A.M. Hillas, in: Proceedings of 15th International Cosmic Ray Conference, Plovdiv, vol. 8, p. 353, 1977.

[205] G. Archbold et al., in: Proceedings of the 28th International Cosmic Ray Conference, Tsukuba, p. 405, 2003.

[206] J. Linsley, in: Proceedings of the 15th International Cosmic Ray Conference, Plovdiv, vol. 12, p. 89, 1977.

[207] J. Linsley, A.A. Watson, Phys. Rev. Lett. 46 (1981) 459.

[208] D.J. Bird et al. (HiRes Collaboration), Phys. Rev. Lett. 71 (1993) 3401.

[209] T. Abu-Zayyad et al. (HiRes Collaboration), Phys. Rev. Lett. 84 (2000) 4276. Available from: <arxiv:astro-ph/9911144>.

[210] T. Abu-Zayyad et al. (HiRes-MIA Collaboration), Astrophys. J. 557 (2001) 686. Available from: $<$ arxiv:astro-ph/0010652>.

[211] F. Arqueros et al. (HEGRA Collaboration), Astron. Astrophys. 359 (2000) 682. Available from: <arxiv:astro-ph/9908202>.

[212] J.W. Fowler, L.F. Fortson, C.C.H. Jui, D.B. Kieda, R.A. Ong, C.L. Pryke, P. Sommers, Astropart. Phys. 15 (2001) 49. Available from: <arxiv:astro-ph/0003190>.

[213] S.P. Swordy, D.B. Kieda, Astropart. Phys. 13 (2000) 137. Available from: <arxiv:astro-ph/ 9909381>.

[214] J.E. Dickinson et al., in: Proceedings of the 26th International Cosmic Ray Conference, Salt Lake City, vol. 3, p. 136, 1999.

[215] S. Knurenko et al., in: Proceedings of the 27th International Cosmic Ray Conference, Hamburg, p. $177,2001$. 
[216] M. Ave, L. Cazon, J.A. Hinton, J. Knapp, J. LLoyd-Evans, A.A. Watson, Astropart. Phys. 19 (2003) 61. Available from: <arxiv:astro-ph/0203150>.

[217] M.T. Dova, M.E. Mancenido, A.G. Mariazzi, T.P. McCauley, A.A. Watson. Available from: $<$ arxiv:astro-ph/0312463>.

[218] M.T. Dova, M.E. Mancenido, A.G. Mariazzi, T.P. McCauley, A.A. Watson. Available from: <arxiv:astro-ph/0305351>.

[219] M.T. Dova, M.E. Mancenido, A.G. Mariazzi, T.P. McCauley, A.A. Watson, Nucl. Phys. Proc. Suppl. 122 (2003) 235. Available from: <arxiv:astro-ph/0210464>.

[220] J. Linsley, in: Proceedings of the 15th International Cosmic Ray Conference, Plovdiv, vol. 12, p. 56, 1977.

[221] J. Linsley, in: Proceedings of the 15th International Cosmic Ray Conference, Plovdiv, vol. 12, p. 52, 1977.

[222] A.A. Watson, J.G. Wilson, J. Phys. A 7 (1974) 1199.

[223] R. Walker, A.A. Watson, J. Phys. G 7 (1981) 1297.

[224] M. Ave, J. Knapp, M.. Marchesini, M. Roth, A.A. Watson, in: Proceedings of the 28th International Cosmic Ray Conference, Tsukuba, p. 349, 2003.

[225] A.A. Watson. Available from: <arxiv:astro-ph/0312475>

[226] C.D. England, PhD Thesis, University of Leeds, UK, 1984.

[227] M.T. Dova (AUGER Collaboration), in: Proceedings of the 28th International Cosmic Ray Conference, Tsukuba, p. 369, 2003. Available from: <arxiv:astro-ph/0308399>.

[228] B.R. Dawson, R. Meyhandan, K.M. Simpson, Astropart. Phys. 9 (1998) 331. Available from: $<$ arxiv:astro-ph/9801260>.

[229] T.K. Gaisser, F. Halzen, T. Stanev, Phys. Rep. 258 (1995) 173 [Erratum-ibid. 271 (1996) 355]. Available from: <arxiv:hep-ph/9410384>.

[230] K.S. Hirata et al. (Kamiokande-II Collaboration), Phys. Rev. D 44 (1991) 2241 [Erratum-ibid. 45 (1992) 2170].

[231] K. Hirata et al. (KAMIOKANDE-II Collaboration), Phys. Rev. Lett. 58 (1987) 1490.

[232] R.M. Bionta, et al., Phys. Rev. Lett. 58 (1987) 1494.

[233] F. Halzen, Phys. Rep. 333 (2000) 349.

[234] F. Halzen, D. Hooper, Rept. Prog. Phys. 65 (2002) 1025. Available from: <arxiv:astro-ph/0204527>.

[235] J.N. Bahcall, M.C. Gonzalez-Garcia, C. Pena-Garay, Phys. Rev. C 66 (2002) 035802. Available from: <arxiv:hep-ph/0204194>.

[236] J.N. Bahcall, V. Barger, D. Marfatia, Phys. Lett. B 534 (2002) 120. Available from: <arxiv:hep-ph/ $0201211>$.

[237] J.F. Beacom, N.F. Bell, D. Hooper, S. Pakvasa, T.J. Weiler, Phys. Rev. Lett. 90 (2003) 181301. Available from: <arxiv:hep-ph/0211305>.

[238] J.F. Beacom, N.F. Bell, D. Hooper, S. Pakvasa, T.J. Weiler, Phys. Rev. D 68 (2003) 093005. Available from: <arxiv:hep-ph/0307025>.

[239] J.F. Beacom, N.F. Bell, D. Hooper, J.G. Learned, S. Pakvasa, T.J. Weiler, Phys. Rev. Lett. 92 (2004) 011101. Available from: <arxiv:hep-ph/0307151>.

[240] J.F. Beacom, N.F. Bell, D. Hooper, S. Pakvasa, T.J. Weiler, Phys. Rev. D 69 (2004) 017303. Available from: <arxiv:hep-ph/0309267>.

[241] L.A. Anchordoqui, H. Goldberg, F. Halzen, T.J. Weiler, Phys. Lett. B 593 (2004) 42.

[242] L.K. Resvanis, Nucl. Phys. Proc. Suppl. 122 (2003) 24.

[243] S.L. Glashow, Phys. Rev. 118 (1960) 316.

[244] R. Gandhi, C. Quigg, M.H. Reno, I. Sarcevic. Available from: <arxiv:hep-ph/9510295>.

[245] R. Gandhi, C. Quigg, M.H. Reno, I. Sarcevic, Astropart. Phys. 5 (1996) 81. Available from: $<$ arxiv:hep-ph/9512364>.

[246] H. Tu, PhD Thesis, DESY, 2004.

[247] H.L. Lai, et al., Phys. Rev. D 55 (1997) 1280. Available from: <arxiv:hep-ph/9606399>.

[248] R. Gandhi, C. Quigg, M.H. Reno, I. Sarcevic, Phys. Rev. D 58 (1998) 093009. Available from: <arxiv:hep-ph/9807264>.

[249] P. Billoir, 8th International Workshop on Neutrino Telescopes, Venice, Italy, 1999. 
[250] G. Domokos, S. Kovesi-Domokos. Available from: <arxiv:hep-ph/9805221>.

[251] D. Fargion, Astrophys. J. 570 (2002) 909. Available from: <arxiv:astro-ph/0002453>.

[252] J.L. Feng, P. Fisher, F. Wilczek, T.M. Yu, Phys. Rev. Lett. 88 (2002) 161102. Available from: $<$ arxiv:hep-ph/0105067>.

[253] X. Bertou, P. Billoir, O. Deligny, C. Lachaud, A. Letessier-Selvon, Astropart. Phys. 17 (2002) 183. Available from: <arxiv:astro-ph/0104452>.

[254] G. Domokos, S. Nussinov, Phys. Lett. B 187 (1987) 372.

[255] J. Bordes, H.M. Chan, J. Faridani, J. Pfaudler, S.T. Tsou. Available from: <arxiv:hep-ph/9705463>.

[256] G. Domokos, S. Kovesi-Domokos, Phys. Rev. Lett. 82 (1999) 1366. Available from: <arxiv:hep-ph/ 9812260>.

[257] Z. Fodor, S.D. Katz, A. Ringwald, H. Tu, Phys. Lett. B 561 (2003) 191. Available from: <arxiv:hep$\mathrm{ph} / 0303080>$.

[258] N. Arkani-Hamed, S. Dimopoulos, G.R. Dvali, Phys. Lett. B 429 (1998) 263. Available from: <arxiv:hep-ph/9803315>.

[259] I. Antoniadis, N. Arkani-Hamed, S. Dimopoulos, G.R. Dvali, Phys. Lett. B 436 (1998) 257. Available from: <arxiv:hep-ph/9804398>.

[260] L. Randall, R. Sundrum, Phys. Rev. Lett. 83 (1999) 3370. Available from: <arxiv:hep-ph/9905221>

[261] S. Hannestad, G.G. Raffelt, Phys. Rev. D 67 (2003) 125008 [Erratum-ibid. 69 (2004) 029901]. Available from: <arxiv:hep-ph/0304029>.

[262] M. Acciarri et al. [L3 Collaboration], Phys. Lett. B 464 (1999) 135. Available from: <arxiv:hep-ex/ 9909019>.

[263] M. Acciarri et al. [L3 Collaboration], Phys. Lett. B 470 (1999) 281. Available from: <arxiv:hep-ex/ 9910056>.

[264] B. Abbott et al. [D0 Collaboration], Phys. Rev. Lett. 86 (2001) 1156. Available from: <arxiv:hep-ex/ 0008065>.

[265] V.M. Abazov et al. [D0 Collaboration], Phys. Rev. Lett. 90 (2003) 251802. Available from: <arxiv:hep-ex/0302014>.

[266] L.A. Anchordoqui, J.L. Feng, H. Goldberg, A.D. Shapere, Phys. Rev. D 68 (2003) 104025. Available from: <arxiv:hep-ph/0307228>.

[267] M. Bando, T. Kugo, T. Noguchi, K. Yoshioka, Phys. Rev. Lett. 83 (1999) 3601. Available from: $<$ arxiv:hep-ph/9906549>.

[268] S. Nussinov, R. Shrock, Phys. Rev. D 59 (1999) 105002. Available from: <arxiv:hep-ph/9811323>.

[269] P. Jain, D.W. McKay, S. Panda, J.P. Ralston, Phys. Lett. B 484 (2000) 267. Available from: <arxiv:hep-ph/0001031>.

[270] L. Anchordoqui, H. Goldberg, T. McCauley, T. Paul, S. Reucroft, J. Swain, Phys. Rev. D 63 (2001) 124009. Available from: <arxiv:hep-ph/0011097>.

[271] M. Kachelriess, M. Plumacher, Phys. Rev. D 62 (2000) 103006. Available from: <arxiv:astro-ph/ 0005309>.

[272] A.V. Kisselev, V.A. Petrov. Available from: <arxiv:hep-ph/0311356>.

[273] T. Banks, W. Fischler. Available from: <arxiv:hep-th/9906038>.

[274] S.B. Giddings, S. Thomas, Phys. Rev. D 65 (2002) 056010. Available from: <arxiv:hep-ph/0106219>.

[275] S. Dimopoulos, G. Landsberg, Phys. Rev. Lett. 87 (2001) 161602. Available from: <arxiv:hep-ph/ 0106295>.

[276] J.L. Feng, A.D. Shapere, Phys. Rev. Lett. 88 (2001) 021303. Available from: <arxiv:hep-ph/ 0109106>.

[277] P. Kanti. Available from: <arxiv:hep-ph/0402168>.

[278] K.S. Thorne, in: J. Klauder (Ed.), Magic Without Magic: John Archibald Wheeler, Freeman, San Francisco, 1972, p. 231.

[279] R.C. Myers, M.J. Perry, Ann. Phys. 172 (1986) 304.

[280] P.C. Argyres, S. Dimopoulos, J. March-Russell, Phys. Lett. B 441 (1998) 96. Available from: <arxiv:hep-th/9808138>.

[281] D.M. Eardley, S.B. Giddings, Phys. Rev. D 66 (2002) 044011. Available from: <arxiv:gr-qc/ 0201034>. 
[282] H. Yoshino, Y. Nambu, Phys. Rev. D 67 (2003) 024009. Available from: <arxiv:gr-qc/0209003>.

[283] J. Pumplin, D.R. Stump, J. Huston, H.L. Lai, P. Nadolsky, W.K. Tung, JHEP 0207 (2002) 012. Available from: <arxiv:hep-ph/0201195>.

[284] D. Stump, J. Huston, J. Pumplin, W.K. Tung, H.L. Lai, S. Kuhlmann, J.F. Owens. Available from: $<$ hep-ph/0303013>.

[285] R. Emparan, M. Masip, R. Rattazzi, Phys. Rev. D 65 (2002) 064023. Available from: <arxiv:hep$\mathrm{ph} / 0109287>$.

[286] R. Emparan, G.T. Horowitz, R.C. Myers, Phys. Rev. Lett. 85 (2000) 499. Available from: $<$ arxiv:hep-th/0003118>.

[287] P. Kanti, J. March-Russell, Phys. Rev. D 66 (2002) 024023. Available from: <arxiv:hep-ph/ $0203223>$.

[288] P. Kanti, J. March-Russell, Phys. Rev. D 67 (2003) 104019. Available from: <arxiv:hep-ph/ 0212199>.

[289] C.M. Harris, P. Kanti, JHEP 0310 (2003) 014. Available from: <arxiv:hep-ph/0309054>.

[290] J. Preskill, P. Schwarz, A.D. Shapere, S. Trivedi, F. Wilczek, Mod. Phys. Lett. A 6 (1991) 2353.

[291] S. Dimopoulos, R. Emparan, Phys. Lett. B 526 (2002) 393. Available from: <arxiv:hep-ph/ 0108060>.

[292] L.A. Anchordoqui, J.L. Feng, H. Goldberg, A.D. Shapere. Available from: <arxiv:hep-ph/ 0309082>.

[293] K.m. Cheung, Phys. Rev. Lett. 88 (2002) 221602. Available from: <arxiv:hep-ph/0110163>.

[294] L. Anchordoqui, H. Goldberg, Phys. Rev. D 67 (2003) 064010. Available from: <arxiv:hep-ph/ 0209337>.

[295] I. Mocioiu, Y. Nara, I. Sarcevic, Phys. Lett. B 557 (2003) 87. Available from: <arxiv:hep-ph/ 0301073>.

[296] A. Chamblin, F. Cooper, G.C. Nayak, Phys. Rev. D 69 (2004) 065010. Available from: <arxiv:hep$\mathrm{ph} / 0301239>$.

[297] L.A. Anchordoqui, J.L. Feng, H. Goldberg, A.D. Shapere, Phys. Lett. B 594 (2004) 363.

[298] A. Chamblin, F. Cooper, G.C. Nayak. Available from: <arxiv:hep-ph/0405054>.

[299] A. Chamblin, G.C. Nayak, Phys. Rev. D 66 (2002) 091901. Available from: <arxiv:hep-ph/ 0206060>.

[300] L. Anchordoqui, H. Goldberg, Phys. Rev. D 65 (2002) 047502. Available from: <arxiv:hep-ph/ 0109242>.

[301] A. Ringwald, H. Tu, Phys. Lett. B 525 (2002) 135. Available from: <arxiv:hep-ph/0111042>.

[302] S.I. Dutta, M.H. Reno, I. Sarcevic, Phys. Rev. D 66 (2002) 033002. Available from: <arxiv:hep-ph/ $0204218>$.

[303] L.A. Anchordoqui, J.L. Feng, H. Goldberg, A.D. Shapere, Phys. Rev. D 66 (2002) 103002. Available from: <arxiv:hep-ph/0207139>.

[304] V. Cardoso, M.C. Espirito Santo, M. Paulos, M. Pimenta, B. Tome. <arxiv:hep-ph/0405056>.

[305] M. Kowalski, A. Ringwald, H. Tu, Phys. Lett. B 529 (2002) 1.

[306] J. Alvarez-Muniz, J.L. Feng, F. Halzen, T. Han, D. Hooper, Phys. Rev. D 65 (2002) 124015.

[307] P. Jain, S. Kar, D.W. McKay, S. Panda, J.P. Ralston, Phys. Rev. D 66 (2002) 065018.

[308] L.A. Anchordoqui, J.L. Feng, H. Goldberg, A.D. Shapere, Phys. Rev. D 65 (2002) 124027. Available from: <arxiv:hep-ph/0112247>. 